\title{
The Monodromy Rings of One Loop Feynman Integrals
}

\author{
G. Ponzano, T. Regge, E. R. SPeer ${ }^{\star}$, and M. J. WestwateR ${ }^{\star}$ \\ Institute for Advanced Study, Princeton, New Jersey
}

Received January 20, 1970

\begin{abstract}
The monodromy rings of Feynman integrals for one loop graphs with an arbitrary number of lines are determined.
\end{abstract}

\section{§ 1. Introduction}

This paper is the second of a series whose general aims were outlined in the introduction to the first paper [1]. In this paper we make a systematic study of the Feynman integral for a general one loop graph in an arbitrary space-time dimension; we classify the possible paths of analytic continuation, label the determinations of the function over a fixed base point, and obtain explicit formulae for the action of analytic continuation on the vector space spanned by these determinations.

A preliminary account of these investigations has been published [2]. The present account is self contained, but for the reader who is familiar with [2], we proceed to give a brief comparison. In the present paper we introduce the auxiliary complex parameters also used in [1] and [3]. This makes a technical difference in the construction of the representation since, for general values of the parameters, the Cutkosky-Steinman relations do not hold; nevertheless, we are able to determine the representation by exploiting more fully the consequences of the PicardLefschetz theorem. Single loop integrals fall naturally into four classes (see § 2). In [2] only case 3 (in which no second kind singularities appear, and the invariants constructed from the external momenta are algebraically independent) was treated. 'Our present use of complex parameters enables us to deal with four cases in a unified manner. Finally, we note that the present paper includes some proofs which were omitted from [2].

We would like to call attention to two points which were raised in the discussion following a talk given by one of us (T. R. [4]). First there is the problem which arises in case 4 - that the scalar invariants of the external momenta are algebraically dependent. We show in this paper

\footnotetext{
${ }^{\star}$ Research sponsored by the National Science Foundation, Grant No. GP-16147.
} 
how the formalism of [2] is adapted to deal with this case. Secondly there is the question as to whether the success of our methods for the single loop integrals depends on their special functional form (they are sums of Spence functions). In our opinion the present calculations, and those of [1], show that the key to the determination of the monodromy of a Feynman integral is the determination of the fundamental group. The fundamental groups of single loop graphs are particularly simple. It is this simplicity (which is determined by the form of the Landau varieties), rather than the functional form of the integrals, which is important. This remark is illustrated by the fact that for general values of the auxiliary parameters single loop integrals are not expressible in terms of Spence functions.

In this paper (as in [1]) we consider all possible analytic continuations i.e. we consider all the parameters which enter into the integrals, including the internal and external masses, as complex variables. It would also be of interest to examine the monodromy representation restricted to analytic continuations along paths in which the internal masses are held constant, or in which both internal and external masses are held constant. Our results give a basis for such investigations but we do not actually deal with this problem.

This paper presupposes the results of $\S 1$ of [1]. We will use the following set-theoretic notations. Let $\chi$ and $\psi$ be any subsets of some universe $\Omega$. Then

a) $\chi-\psi=\{x \mid x \in \chi, x \notin \psi\}$ (note we do not assume $\psi \subset \chi$ );

b) $\chi \Delta \psi=(\chi-\psi) \cup(\psi-\chi)=$ the symmetric difference of $\chi$ and $\psi$;

c) $|\chi|=$ the number of elements in $\chi$;

d) $\chi^{\prime}=\Omega-\chi$;

e) $\delta_{\psi \subset \chi}= \begin{cases}1, & \text { if } \psi \subset \chi, \\ 0, & \text { otherwise . }\end{cases}$

We will sometimes ignore the distinction between an element $x \in \Omega$ and the one-element set $\{x\}$.

\section{§ 2. Definition of the Integrals}

We denote by $G_{N}$ the single loop graph with $N \geqq 2$ vertices. The internal and external lines of $G_{N}$ we label as in Fig. 1. The dimension of space-time will be given an arbitrary value $m \geqq 2$. For the $i$ th external line we are given a momentum vector $k_{i, i+1} \in \mathbb{R}^{m}$, these satisfy the conservation condition

$$
\sum_{i=1}^{N} k_{i, i+1}=0 .
$$


In view of (2.1), we may introduce vectors $k_{j} \in \mathbb{R}^{m}, j=1, \ldots, N$, such that $k_{i, i+1}=k_{i}-k_{i+1}$. (In these equations, it is understood that $k_{N, N+1}$ and $k_{N+1}$ are to be replaced by $k_{N, 1}$ and $k_{1}$ respectively.) Then we will study the integral

$$
F\left(k_{i, i+1}, m_{j}^{2}\right)=\int_{\mathbb{R}^{m}} d^{m} k \prod_{j=1}^{N}\left[\left(k+k_{j}\right)^{2}-m_{j}^{2}\right]^{-1},
$$

where $m_{j} \in \mathbb{R}^{1}$ is the mass of the $j$ th internal line, and

$$
p^{2}=-\sum_{i=1}^{m} p_{i}^{2},
$$

for any $p \in \mathbb{R}^{m}$. For $N \leqq m / 2$ this integral diverges and must be renormalized (see 5.4). Otherwise it defines a function analytic for real values of its arguments; this function may be extended to a (many-valued) function in the complex domain, whose monodromy ring we wish to study. The relation of this Euclidean integral to physically relevant quantities will be discussed in 5.1.

It is convenient to write $F$ in a parametric representation [5] which displays its dependence on invariants. Up to unimportant factors,

where

$$
F(A)=\Gamma(N-m / 2) \int_{\alpha_{i} \geqq 0} \prod_{i=1}^{N} d \alpha_{i} \delta\left(1-\sum_{1}^{N} \alpha_{i}\right) \frac{[d(\alpha)]^{N-m}}{[D(\alpha, A)]^{N-m / 2}},
$$

$$
\begin{gathered}
d(\alpha)=\sum_{i=1}^{N} \alpha_{i}, \\
D(\alpha, A)=\sum_{i, j=1}^{N} A_{i j} \alpha_{i} \alpha_{j},
\end{gathered}
$$

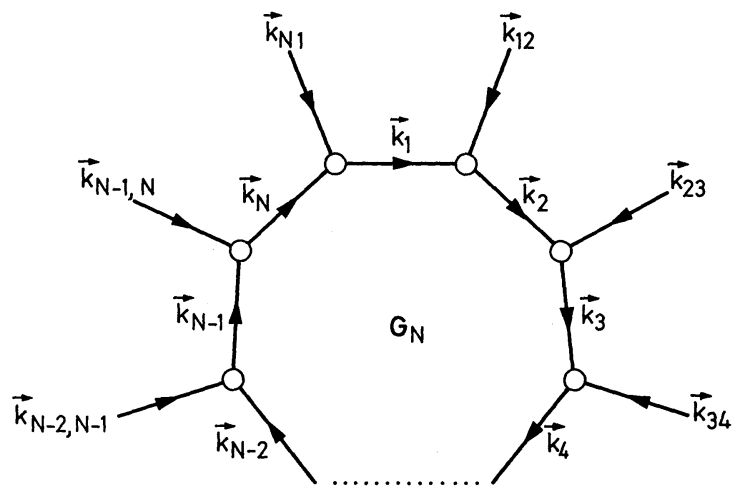

Fig. 1. The single loop graph $G_{N}$ 
and

$$
\begin{aligned}
& A_{i i}=m_{i}^{2}, \\
& A_{i j}=A_{j i}=\frac{1}{2}\left[m_{i}^{2}+m_{j}^{2}-\left(k_{j}-k_{i}\right)^{2}\right] .
\end{aligned}
$$

For the moment we will consider $F$ as a function of the $N(N+1) / 2$ complex variables $A_{i j}$. Note, however, that for $N>m+1$ these invariants are not algebraically independent. It is then more convenient to study (2.2) directly in momentum space; this problem will be taken up in $\S 6$.

To obtain the singular locus $L$ of $F$, we rewrite (2.3) as a projective integral. Up to a sign,

$$
F(A)=\Gamma(N-m / 2) \int_{\substack{\alpha_{i} \geqq 0, i=1, \ldots N \\-\infty<\alpha_{N+1}<\infty}} \delta\left(\sum_{i=1}^{N+1} \alpha_{i}\right) \frac{\alpha_{N+1}^{N-m}}{D(\alpha, A)^{N-m / 2}}{ }^{N} \eta,
$$

where ${ }^{N} \eta$ is the fundamental projective form [6]

$$
{ }^{N} \eta=\sum_{i=1}^{N+1}(-1)^{i} \alpha_{i} d \alpha_{1} \ldots \widehat{d \alpha_{i}} \ldots d \alpha_{N+1} .
$$

The singularities of (2.4) depend on whether $\left\{\alpha_{N+1}=0\right\}$ is a zero or pole of the integrand. We are thus led to distinguish four cases in our study of (2.2):

1. $N \leqq m / 2$. The integral (2.2) is divergent, as reflected by the pole of the $\Gamma$-function in (2.4). This case will be handled by analytic renormalization (5.4); however, the location of singularities is the same as case 2.

2. $m / 2<N<m$. The factor $\alpha_{N+1}$ in (2.4) introduces second kind singularities [5].

3. $m \leqq N \leqq m+1$. There are no second kind singularities, and the invariants $A_{i j}$ are independent.

4. $N>m+1$. As remarked above, the integral in this case will be studied, in momentum space, in $\S 6$.

All the above cases may be obtained by suitable specialization of the integral

$$
H_{\gamma}\left(B_{i j}\right)=\int_{\gamma} \frac{P(x) \prod_{j=1}^{r}\left[l_{j}(x)\right]^{\lambda_{j}}}{[B(x)]^{\mu}} s \eta(x) .
$$

In (2.5) $P(x)$ is a fixed homogeneous polynomial of degree $p$ in $x_{1}, \ldots, x_{s+1}$; $l_{j}(x), 1 \leqq j \leqq r$, is a fixed linear homogeneous polynomial in $x ; B(x)$ is given by

$$
B(x)=\sum_{i, j=1}^{s+1} B_{i j} x_{i} x_{j}
$$


$\lambda_{j}, 1 \leqq j \leqq r$, and $\mu$ are complex numbers satisfying

$$
p+\sum_{j=1}^{r} \lambda_{j}+(s+1)=2 \mu ;
$$

and $\gamma$ is an arbitrary cycle on which the integrand is single-valued. We will study this general integral in $\S 3$ and $\S 4$ and apply our results to the physical cases in $\S 5$ and $\S 6$.

As in our preceding paper [1] we consider Feynman amplitudes for spin zero particles; the results of $\S 3$ and $\S 4$ may also be applied to the case in which the exchanged particles have non-zero spin. We note that it is then possible to have amplitudes with second kind singularities for which the invariants are algebraically dependent.

\section{§ 3. The Fundamental Group $\mathscr{G}(r, s)$}

In this section and the following section we determine the monodromy ring of the function $H$ introduced at the end of $\S 2$, considering $H$ as a function on the space $W=\mathbb{C}^{(s+1)(s+2) / 2}$ of quadratic forms $B$. This section is devoted to the study of the fundamental group of the complement of the singular variety of $H$. In $\S 4$ we formulate the additional conditions on the monodromy representation of this group derived from a local analysis in the integration space, and use them to construct explicitly the monodromy ring of $H$.

\subsection{The Singular Variety $M$}

We will suppose throughout that the linear forms $l_{j}$ in (2.5) satisfy the condition that any $k \leqq s+1$ of them are linearly independent. We denote by $\Omega$ the set $\{1, \ldots, r\}$ and we use Greek letters to denote subsets of $\Omega$. For any $\chi \subset \Omega, 0 \leqq|\chi| \leqq s$, write

$$
l(\chi)=\left\{x \mid l_{j}(x)=0, \forall_{j} \in \chi\right\} \subset \mathbb{P}^{s} .
$$

Then the algebraically irreducible components of the singular variety $M$ of $H$ are the varieties $M(\chi)$ defined by

$$
M(\chi)=\left\{B|B(x)|_{l_{(\chi)}} \text { is a singular quadratic form }\right\} .
$$

The defining condition (3.1) may be expressed in terms of coordinates as follows:

$B \in M(\chi)$ iff the equation

$$
\left.\begin{array}{rl}
l_{j}(x) & =0, \quad \forall_{j} \in \chi, \\
\frac{\partial B}{\partial x_{k}}+\sum_{j \in \chi} \tau_{j}(B, \chi) \frac{\partial l_{j}}{\partial x_{k}} & =0, \text { for } 1 \leqq k \leqq s+1,
\end{array}\right\}
$$


have a solution $x=x(B, \chi) \in \mathbb{P}^{s}$. Here the $\tau_{j}$ are Lagrange multipliers. The set of solutions $x(B, \chi)$ is called the pinch in the integration space corresponding to $B$. These equations assume a particularly simple form if we choose the coordinate system so that the $l_{j}(x), j \in \chi$, are the first $|\chi|$ coordinate variables (which is possible in view of the condition imposed at the beginning of this subsection).

$$
\left.\begin{array}{rl}
x_{j} & =0, \quad 1 \leqq j \leqq|\chi|, \\
\frac{\partial B}{\partial x_{k}} & =0, \quad|\chi|+1 \leqq k \leqq s+1, \\
\tau_{j}(B, \chi) & =-\frac{\partial B}{\partial x_{j}}, j \in \chi .
\end{array}\right\}
$$

The Eq. (3.1.3) have a solution iff the determinant of the symmetric matrix $B(\chi)$ formed by the last $s+1-|\chi|$ rows and columns of the coefficient matrix of $B$ (in this coordinate system) vanishes. This gives us a global equation for $M(\chi)$ which is therefore an algebraic variety of degree $s+1-|\chi|$.

We now consider the singular points of $M=\bigcup_{\chi} M(\chi)$ as an algebraic variety. Let $B \in M(\chi)$ and choose coordinates in $\mathbb{P}^{s}$ as in the preceding paragraph. If $B(\chi)$ has nullity 1 the solution $x(B, \chi)$ of $(3.1 .3)$ is uniquely determined by $B$ and $\chi$; in fact

$$
\begin{aligned}
(\operatorname{adj} B(\chi))_{k l}= & x_{k}(B, \chi) x_{l}(B, \chi) \\
& |\chi|+1 \leqq k, l \leqq s+1 .
\end{aligned}
$$

We may define $\operatorname{adj} B(\chi)$ in an arbitrary coordinate system as the matrix of coefficients of the tangent variety to

$$
B(\chi)=\left\{(B) \mid B(x)=0, \quad l_{j}(x)=0, \quad \forall_{j} \in \chi\right\},
$$

and write (3.1.4) in coordinate-free form

$$
\operatorname{adj} B(\chi)=x(B, \chi) \otimes x(B, \chi) .
$$

For the normal to $M(\chi)$ at $B$ we obtain

$$
\begin{aligned}
\frac{\partial M(\chi)}{\partial B_{i j}} & =\frac{\partial}{\partial B_{i j}}(\operatorname{det} B(\chi))=2(\operatorname{adj} B(\chi))_{i j} \\
& =2 x_{i}(B, \chi) x_{j}(B, \chi),
\end{aligned}
$$

for $|\chi|+1 \leqq i, j \leqq s+1$,

$$
=0 \text { otherwise; }
$$


or in coordinate-free form

$$
\frac{\partial}{\partial B} M(\chi)=2 x(B, \chi) \otimes x(B, \chi) .
$$

According to (3.1.6) $B$ is a nonsingular point of $M(\chi)$. If $B(\chi)$ has nullity $n \geqq 2, \operatorname{adj} B(\chi)=0$ and

$$
\frac{\partial M(\chi)}{\partial B_{i j}}=0, \quad \forall_{i, j},
$$

so that $M(\chi)$ is singular. However, a simple counting of conditions shows that

$$
M(\chi, n)=\{B \mid B(\chi) \text { has nullity } \geqq n\}
$$

has complex codimension $n(n+1) / 2$ in $W$. In particular $M(\chi, 2)$ has codimension 3 (if it is non-empty).

Now suppose that for two distinct subsets $\chi, \psi$ of $\Omega$

$$
B \in(M(\chi)-M(\chi, 2)) \cap(M(\psi)-M(\psi, 2)) .
$$

Then either the points $x(B, \chi), x(B, \psi)$ are distinct and so by (3.1.6) $M(\chi)$ and $M(\psi)$ intersect transversely at $B$, or $x(B, \chi)=x(B, \psi)$ and by (3.1.6) $M(\chi)$ and $M(\psi)$ have a common tangent at $B$. In the latter case, this common point $x=x(B, \psi)=x(B, \chi)$ must satisfy the equations

$$
\begin{aligned}
l_{j}(x) & =0, \quad j \in \chi \cup \psi, \\
\left.\frac{\partial B}{\partial x_{k}}\right|_{x} & =0, \quad k \notin \chi \cap \psi,
\end{aligned}
$$

where we have used a coordinate system in which $l_{j}(y)=y_{j}, j \in \chi \cup \psi$. These equations imply that $B \in M(\varrho)$ for any $\chi \cap \psi \subset \varrho \subset \chi \cup \psi$. Now if the symmetric difference of $\chi$ and $\psi$ contains at least two elements, the Eqs. (3.1.8) imply at least 3 conditions on the coefficients $B_{i j}$; thus the set of such singular points has codimension $\geqq 3$. Otherwise, i.e. if $\chi=\psi \cup\{i\}$ or $\psi=\chi \cup\{i\}$, the singular set has codimension 2 and there are precisely two components of $M$ tangent at $B$.

\subsection{The Real Points of $M$}

In this section we study the real part $M_{R}$ of $M$, that is, the points of $M$ for which the quadratic form $B$ has real coefficients. Let $S_{R} \subset M_{R}$ be the set of all real singular points of $M$ except for those which correspond to a transverse intersection of regular components of $M$. The fundamental group $\mathscr{G}(r, s)$ will be described in terms of elementary loops around 
points of $M_{R}-S_{R}$; in this section we begin this description by labelling the connected components of $M_{R}-S_{R}$.

The labelling we use refers to the pinch which generates the singular point. For each $B \in M(\chi) \cap\left[M_{R}-S_{R}\right]$ we define two $r$-dimensional vectors given by

$$
\left.\begin{array}{l}
l_{j}[x(B, \chi)], \\
\tau_{j}(B, \chi),
\end{array}\right\} \quad j=1, \ldots r
$$

(see 3.1.2), where we write $\tau_{j}(B, \chi) \equiv 0$ for $j \notin \chi$. Then the point $B$ is labelled by the signature

where

$$
\mathscr{S}=\left(\begin{array}{ll}
\varrho_{1} & \varrho_{4} \\
\varrho_{3} & \varrho_{2}
\end{array}\right)
$$

$$
\begin{aligned}
& \varrho_{1}=\left\{j \in \Omega \mid l_{j}[x(B, \chi)]>0\right\}, \\
& \varrho_{2}=\left\{j \in \Omega \mid l_{j}[x(B, \chi)]<0\right\}, \\
& \varrho_{3}=\left\{j \in \Omega \mid \tau_{j}(B, \chi)>0\right\}, \\
& \varrho_{4}=\left\{j \in \Omega \mid \tau_{j}(B, \chi)<0\right\} .
\end{aligned}
$$

The sets $\varrho_{1}, \ldots \varrho_{4}$ form a partition of $\Omega$, with $\varrho_{1} \cup \varrho_{2}=\Omega-\chi, \varrho_{3} \cup \varrho_{4}=\chi$ (for if $l_{j}[x(B, \chi)]=0, j \notin \chi$, or $\tau_{j}(B, \chi)=0, j \in \chi$, the point $B$ must lie in $S_{R}$ ). The reason for displaying the signature in the form (3.2.1) will become apparent when we examine the algebraic relations in $\mathscr{G}(r, s)$. The freedom to change projective coordinates by a factor means that we must identify with (3.2.1) the signature obtained from it by interchanging $\varrho_{1}$ with $\varrho_{2}$ and $\varrho_{3}$ with $\varrho_{4}$.

Finally we label each point $B \in M(\chi) \cap\left\{M_{R}-S_{R}\right\}$ by a pair $(\mathscr{S}, q)$, where $\mathscr{S}$ is the signature of the point and $q$ is the number of positive eigenvalues of the matrix $B(\chi)$. Note that a point on the transverse intersection of $M(\chi)$ and $M(\psi)$ will have separate labels corresponding to $\chi$ and $\psi$. We define

$$
M(\mathscr{S}, q) \equiv\left\{B \in M(\chi) \cap\left\{M_{R}-S_{R}\right\} \mid B \text { has label }(\mathscr{S}, q)\right\} ;
$$

note that the set $\chi$ is determined by the signature $\mathscr{S}$, specifically, $\chi=\varrho_{3} \cup \varrho_{4}$ when $\mathscr{S}$ is given by (3.2.1).

Lemma 3.2.2. $M(\mathscr{S}, q)$ is pathwise connected.

Proof. Let $B, B^{\prime}$ be two points in $M(\mathscr{S}, q) \subset M(\chi)$. We have to construct a path from $B$ to $B^{\prime}$ lying entirely in $M(\mathscr{S}, q)$. Choose coordinates such that $l_{j}(x)=x_{j}$ for $j \in \chi$ and denote as before by $B(\chi)$ the submatrix of $B$ 
formed by the rows and columns whose indices are not in $\chi$. Then for $B \in M(\mathscr{S}, q), B(\chi)$ has one zero and $q$ positive eigenvalues. We will construct the path as the union of three segments.

1. Choose an orthogonal metric in the space $\mathbb{C}^{s+1-|x|}$ of coordinates $x_{j}, j \notin \chi$. Denote by $x(B, \chi), x\left(B^{\prime}, \chi\right)$ the null vectors of $B(\chi), B^{\prime}(\chi)$, normalized to unit length, and normalized in sign so that

$$
\operatorname{sgn} l_{j}(x(B, \chi))=\operatorname{sgn} l_{j}\left(x\left(B^{\prime}, \chi\right)\right), \quad \forall_{j} \notin \chi .
$$

Let $x(t)=\left[(1-t) x(B, \chi)+t x\left(B^{\prime}, \chi\right)\right] /\left\|(1-t) x(B, \chi)+t x\left(B^{\prime}, \chi\right)\right\|$ for $0 \leqq t \leqq 1$. Then $\operatorname{sgn} l_{j}(x(t))$ is independent of $t$ and there is an orthogonal transformation $O(t) \in O_{+}(s+1-|\chi|)$, the proper orthogonal group, which maps $x(0)$ into $x(t)$ and leaves the subspace orthogonal to the span of $x(0), x(1)$ invariant. The first segment of our path is defined by matrices $B(t)$ such that $B(\chi)(t)=O(t) B(\chi) O(t)^{-1}$. The submatrices $B(\chi)(t)$ have the same eigenvectors as $B(\chi)$ so they are of nullity one and have $q$ positive eigenvalues. To ensure that $B(t) \in M(\mathscr{S}, q)$ for $0 \leqq t \leqq 1$ we choose the components $B_{j k}(t)$ of $B(t)$ with $j \in \chi, k \notin \chi$ so that $\tau_{j}(B(t), \chi)$ $=-\left.\frac{\partial B(t)}{\partial x_{j}}\right|_{x(t)}$ is independent of $t$; this is easy since this condition imposes linear constraints on the choice of these coefficients. Finally we define $B_{j k}(t)=B_{j k}$ for $j, k \in \chi$.

2. The matrices $B(\chi)(1)$ and $B^{\prime}(\chi)$ have a common null vector $x(1)$ and the same number of positive eigenvalues. Thus there is a path $O(t) \in O_{+}(s+1-|\chi|), 1 \leqq t \leqq 2$, such that $O(1)$ is the identity, $O(t)$ leaves $x(1)$ invariant, and $O(2)$ carries any eigenvector of $B(\chi)(1)$ into an eigenvector of $B^{\prime}(\chi)$ whose eigenvalue has the same sign. Then we define $B(t), 1 \leqq t \leqq 2$, by

$$
\begin{aligned}
B(\chi)(t) & =O(t) B(\chi)(1) O(t)^{-1}, \\
B_{i j}(t) & =B_{i j}(1), \quad i \text { or } j \in \chi .
\end{aligned}
$$

3. The matrices $B(\chi)(2)$ and $B^{\prime}(\chi)$ have common eigenvectors, and the signs of the respective eigenvalues agree. Thus we may define $B(t)$, $2 \leqq t \leqq 3$, by

$$
B(t)=(3-t) B(2)+(t-2) B^{\prime} .
$$

Along this path all eigenvectors of $B(\chi)(t)$ are constant, all eigenvalues of $B(\chi)(t)$ retain their sign, and the vectors $\tau_{j}(j \in \chi)$, which for a fixed null vector depend linearly on the coefficients $B_{i j}(t), i \in \chi, j \notin \chi$, also retain their sign. Thus the signature $\mathscr{S}$ and the number $q$ are constant along the entire path. 


\subsection{Construction of the Group}

In this section we choose a base point in the parameter space, and define a set of elementary loops on this base point which generates the fundamental group $\mathscr{G}(r, s)$. These generators are elementary loops around the components of $M_{R}-S_{R}$ which were labelled in the previous section. We also derive relations between these generators which are obtained from homotopies in the neighborhood of the real section; although we do not prove that these exhaust the relations in the group, they do suffice to construct the representation.

We choose as base point a positive definite quadratic form $B_{0}$. Consider a real line through $B_{0}$. A point on this line is given parametrically by

$$
B=\lambda B_{1}-B_{0}
$$

where $B_{1} \neq B_{0}$ is some real point in the parameter space. The intersections of this line with $M(\chi)$ are then given by the eigenvalue equation

$$
\operatorname{det}\left(\lambda B_{1}(\chi)-B_{0}(\chi)\right)=0 \text {. }
$$

Since $B_{0}(\chi)$ is positive definite and $B_{1}(\chi)$ is symmetric we have

Lemma 3.3.2. All the intersections of a real line through $B_{0}$ with $M$ are real.

The roots of (3.3.1) can never vanish; we may therefore denote their reciprocals by $\mu_{1}(\chi), \ldots, \mu_{s+1-|\chi|}(\chi)$, and assume that

$$
\mu_{1}(\chi) \leqq \cdots \leqq \mu_{s+1-|\chi|}(\chi) .
$$

Corollary 3.3.3. The fundamental group

$$
\mathscr{G}(r, s)=\pi_{1}\left(W-M ; B_{0}\right)
$$

is generated by elementary loops around points of $M_{R}-S_{R}$.

Proof. This follows from the Picard-Severi theorem (cited in [1]). We obtain certain restrictions on the order in which the points of intersection may appear along a real line through $B_{0}$ from the Courant minimax principle ([7]):

Lemma 3.3.4. (Courant minimax principle). Let $Q$ be a real quadratic form on $\mathbb{R}^{m}$; let $\mathbb{R}^{l}$ be an arbitrary subspace of $\mathbb{R}^{m}$ of dimension $l \leqq m$ and define the bound

$$
C\left(Q, \mathbb{R}^{l}\right)=\sup _{x \in \mathbb{R}^{l}} \frac{\left(x^{T} Q x\right)}{x^{T} x} .
$$

Then $\mu_{l}=\inf _{\mathbb{R}^{l} \subset \mathbb{R}^{m}} C\left(Q, \mathbb{R}^{l}\right), l=1,2, \ldots m$, are the eigenvalues of $Q$ arranged in non-decreasing order. 
Now we can prove

Lemma 3.3.5. Suppose $\chi \subset \psi \subset \Omega$, with $|\psi| \leqq s$. Then for $1 \leqq p \leqq s+1-|\psi|$,

$$
\mu_{p}(\chi) \leqq \mu_{p}(\psi) \leqq \mu_{p+|\psi-\chi|}(\chi) .
$$

Proof. Set $Q=B_{0}^{-\frac{1}{2}} B_{1} B_{0}^{-\frac{1}{2}}$. By 3.3.4

$$
\begin{aligned}
& \mu_{p}(\chi)=\inf _{\mathbb{R}^{p} \subset l(\chi)} \sup _{x \in \mathbb{R}^{p}} \frac{x^{T} Q x}{\left(x^{T} x\right)} \\
& \leqq \inf _{\mathbb{R}^{p} \subset l(\psi)} \sup _{x \in \mathbb{R}^{p}} \frac{x^{T} Q x}{x^{T} x}=\mu_{p}(\psi)
\end{aligned}
$$

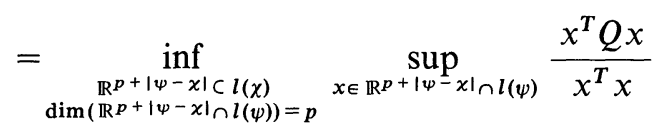

$$
\begin{aligned}
& =\inf _{\mathbb{R}^{p+|\psi-x| c l(x)}} \sup _{x \in \mathbb{R}^{p+|\psi-\chi| \cap l(\psi)}} \frac{x^{T} Q x}{x^{T} x} \\
& \leqq \inf _{\mathbb{R}^{p+|\psi-\chi| \subset}(l(\chi)} \sup _{x \in \mathbb{R}^{p+\lceil\psi-\chi \mid}} \frac{x^{T} Q x}{x^{T} x}=\mu_{p+|\psi-\chi|}(\chi) .
\end{aligned}
$$

Here the first step follows immediately from the inclusion $l(\psi) \subset l(\chi)$, the third from the fact that $\mathbb{R}^{p+|\psi-\chi|} \cap l(\psi)$ has dimension $\geqq p$.

We recall the anticlockwise convention for constructing elementary loops around real points of a Landau variety established in $\S 1.2$ of [1]. The singular points of $M$ in $M(\mathscr{S}, q)$ are transverse intersections so that by Table $1, \S 1.2$ of [1], the elementary loops around points of $M(\mathscr{S}, q)$ on either side of one of these intersections are homotopic. Lemma 3.3.2 then implies that the homotopy class $\alpha(\mathscr{S}, q)$ of an elementary loop constructed according to the anticlockwise convention around a point $B \in M(\mathscr{S}, q)$ depends only on $\mathscr{S}$ and $q$. We note that not every partition of $\Omega$ may be realized as the label $\mathscr{S}$ of some component of $M_{R}-S_{R}$ as in (3.2.1). However, we have

Lemma 3.3.6. If $\alpha(\mathscr{S}, q)$ is defined for a given partition $\mathscr{S}=\left(\begin{array}{ll}\varrho_{1} & \varrho_{4} \\ \varrho_{3} & \varrho_{2}\end{array}\right)$ of $\Omega$ and a given integer $q$ then $\alpha\left(\mathscr{S}, q^{\prime}\right)$ is defined for all $q^{\prime}, 0 \leqq q^{\prime} \leqq s-\left|\varrho_{3}\right|$ $-\left|\varrho_{4}\right|$, and

$$
\alpha\left(\mathscr{S}, q^{\prime}\right)=\alpha(\mathscr{S}, q) \equiv \alpha(\mathscr{S}) .
$$

Proof. It suffices to consider the case $q^{\prime}=q-1$. Let $B$ be a point in $M(\mathscr{S}, q) \subset M(\chi)$ for $\chi=\varrho_{3} \cup \varrho_{4}$. Denote by $B^{\prime}$ a matrix such that:

1. $B_{i j}^{\prime}=B_{i j}$, if $i$ or $j$ is in $\chi$; 
2. $B^{\prime}(\chi)$ is of nullity 1 , having the same eigenvectors as $B(\chi)$. The corresponding eigenvalues are the same as those of $B(\chi)$ except for one negative eigenvalue corresponding to a positive eigenvalue of $B(\chi)$.

Then $B^{\prime} \in M(\mathscr{S}, q-1)$ and the line segment joining $B$ to $B^{\prime}$ passes through just one singular point of $M$, which is a point of $M(\chi, 2)$. According to a result of Byers and Yang [8] this will be a conical point of $M(\chi)$. Fig. 2 shows the plane section $B_{0} B B^{\prime}$ of the parameter space. Lemma 3.3.5 implies that $B_{0}$ lies in the angular sector bounded by the generators of the cone lying in $B_{0} B B^{\prime}$ and we obtain immediately from the identification relations for the conical point the desired relation $\alpha(\mathscr{S}, q)=\alpha(\mathscr{S}, q-1)$.

We now study the question of what partitions $\varrho_{1}, \ldots, \varrho_{4}$ of $\Omega$ may be realized as signatures. This is determined by the relations among the linear forms $l_{j}$.

Definition 3.3.7. Suppose $r \geqq s+1$. Write $l_{j}(x)=\sum_{k=1}^{s+1} l_{j k} x_{k}$, and define the positional characteristic of the linear forms $l_{j}$ by

$$
\left(i_{1} \ldots, i_{s+1}\right)=\operatorname{sgn}\left\{\begin{array}{lll}
l_{i_{1} 1} & \ldots & l_{i_{1} s+1} \\
\vdots & & \\
i_{i_{s+1}} & \ldots & l_{i_{s+1}}+1
\end{array} \mid\right\} .
$$

for any $i_{1}, \ldots, i_{s+1} \in\{1, \ldots r\}$. From our basic linear independence condition on the $l_{j},\left(i_{1} \ldots i_{s+1}\right) \neq 0$ for distinct indices $i_{1}, \ldots i_{s+1}$.

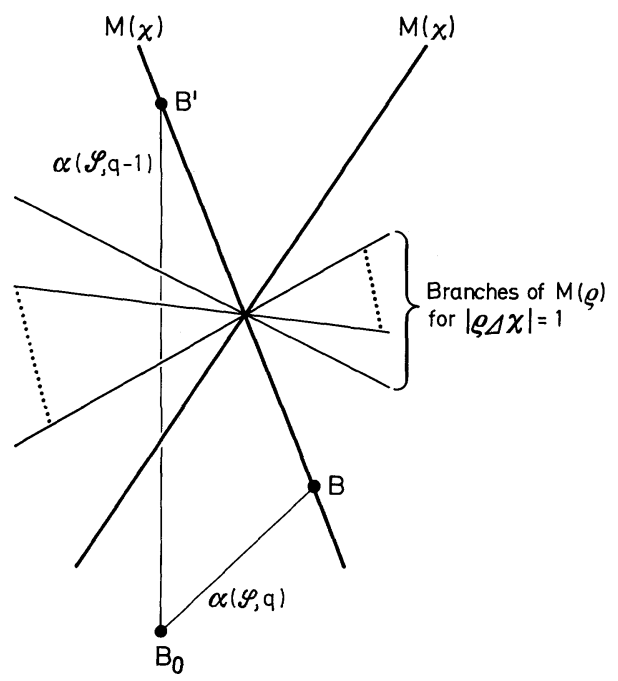

Fig. 2. A section through a conical point 
Definition 3.3.8. Let $\mathscr{S}=\left(\begin{array}{ll}\varrho_{1} & \varrho_{4} \\ \varrho_{3} & \varrho_{2}\end{array}\right)$ be any partition of $\Omega$. The transpose of $\mathscr{S}, \mathscr{S}^{T}$, is the partition $\mathscr{S}^{T}=\left(\begin{array}{ll}\varrho_{2} & \varrho_{3} \\ \varrho_{4} & \varrho_{1}\end{array}\right)$. An array $\mathscr{T}=\left(\begin{array}{ll}\eta_{1} & \eta_{4} \\ \eta_{3} & \eta_{2}\end{array}\right)$, $\eta_{i} \subset \Omega$, is a reduced partition of $\mathscr{S}$ if $\eta_{i} \subset \varrho_{i}, i=1, \ldots 4$.

Lemma 3.3.9. If $r \leqq s$, then every partition of $\Omega$ may be realized as a signature. Suppose $r \geqq s+1$, and define, for any distinct $i_{1}, \ldots i_{s} \in\{1 \ldots r\}$, $\mathscr{T}_{i_{1} \ldots i_{s}}=\left(\begin{array}{ll}\eta_{1} & \phi \\ \phi & \eta_{2}\end{array}\right)$, with

$$
\begin{aligned}
& \eta_{1}=\left\{1 \leqq j \leqq r \mid\left(i_{1}, \ldots i_{s}, j\right)>0\right\} \\
& \eta_{2}=\left\{1 \leqq j \leqq r \mid\left(i_{1}, \ldots i_{s}, j\right)<0\right\} .
\end{aligned}
$$

Then a partition $\mathscr{S}$ of $\Omega$ is admissible (i.e., occurs as a signature) iff, for some $i_{1} \ldots i_{s}, \mathscr{T}_{i_{1} \ldots i_{s}}$ is a reduced partition of $\mathscr{S}$ or of $\mathscr{S}^{T}$.

Proof. The case $r \leqq s$ is easy. Suppose then that $r \geqq s+1$ and that $\mathscr{S}$ is admissible. Let $B$ be a point in $M(\mathscr{S})=\bigcup_{q} M(\mathscr{S}, q)$, and denote as before by $x(B, \chi)$ the corresponding point in $\mathbb{P}^{s}$. Suppose first $|\chi|=s$. Then $x(B, \chi)$ is uniquely determined by the equations $l_{j}(x(B, \chi))=0, j \in \chi$. Solving these equations we find that the coordinates $x(B, \chi)_{k}$ of $x(B, \chi)$ are the cofactors of the elements $\xi_{k}$ in the determinant

$$
\left|\begin{array}{lll}
\xi_{1} & \ldots & \xi_{s+1} \\
l_{i_{1} 1} & \ldots & l_{i_{1}+1} \\
\vdots & & \vdots \\
l_{i_{s} 1} & \ldots & l_{i_{s} s+1}
\end{array}\right|
$$

where $\chi=\left\{i_{1}, \ldots, i_{s}\right\}$. Thus for $j \notin \chi$ we have

$$
l_{j}(x(B, \chi))=\left|\begin{array}{lll}
l_{j 1} & \ldots & l_{j s+1} \\
l_{i_{1} 1} & \ldots & l_{i_{1} s+1} \\
\vdots & & \vdots \\
i_{i_{s} 1} & \ldots & l_{i_{s} s+1}
\end{array}\right|,
$$

and the resulting equality of the signs implies that $\mathscr{T}_{i_{1} \ldots i_{s}}$ is a reduced partition of $\mathscr{S}$ or $\mathscr{S}^{T}$.

Now consider the case $|\chi|<s$. Then $x(B, \chi) \in l(\chi)$ lies in the interior of a region in $l(\chi)$ bounded by certain of the hyperplanes $l(j)$ for $j \notin \chi$. Choose a vertex $y$ on the boundary of this region. Then $y \in l(\psi)$ for some $\psi \supset \chi$ with $|\psi|=s$ and for any $j \notin \psi$ we have

$$
\operatorname{sgn} l_{j}(y)=\operatorname{sgn} l_{j}(x(B, \chi)) .
$$


This equality of signs and the relation of $l_{j}(y), j \notin \psi$, to the positional characteristic of the $l_{j}$ noted already in the case $|\chi|=s$ imply that $\mathscr{T}_{\psi}$ is a reduced partition of $\mathscr{S}$ or $\mathscr{S}^{T}$.

Suppose conversely that a set $\chi=\left\{i_{1} \ldots i_{s}\right\}$ of distinct indices is given, together with a partition $\mathscr{S}=\left(\begin{array}{ll}\varrho_{1} & \varrho_{4} \\ \varrho_{3} & \varrho_{2}\end{array}\right)$ of $\Omega$ having $\mathscr{T}_{i_{1} \ldots i_{s}}$ as a reduced partition. Let $\psi=\varrho_{3} \cup \varrho_{4}$; then $\psi \subset \chi$ and in the neighbourhood of the unique point $y \in l(\chi)$ there exists a point $x \in l(\psi)$ such that $\operatorname{sgn} l_{j}(x)$ is positive for $j \in \chi \cap \varrho_{1}$, negative for $j \in \chi \cap \varrho_{2}$. Furthermore for $j \notin \chi \operatorname{sgn} l_{j}(x)$ $=\operatorname{sgn} l_{j}(y)=\left(j i_{1} \ldots i_{s}\right)$; since $\mathscr{T}_{\chi}$ is a reduced partition of $\mathscr{S}$ it follows that $\operatorname{sgn} l_{j}(x)$ is positive for all $j \in \varrho_{1}$, negative for all $j \in \varrho_{2}$. We can now construct a $B \in W$ such that $B(\psi)$ has $x$ as null vector, and having components $B_{i j}, i \in \psi, j \notin \psi$, chosen so that the $\tau_{j}, j \in \psi$, which are given by

$$
\tau_{j}=\sum_{k \notin \psi} B_{j k} x_{k},
$$

have the signs specified by $\varrho_{3}$ and $\varrho_{4}$.

We now derive the van Kampen relations satisfied by the generators $\alpha(\mathscr{S})$ of $\mathscr{G}(r, s)$ (see [1] for a discussion of these relations and their derivations). We first discuss the bicommutation relations arising from the tacnodal contact of $M(\chi)$ and $M(\chi \cup i)$ (3.1).

Lemma 3.3.10. Let $B_{1}$ be a generic point of $M(\chi) \cap M(\chi \cup i)$. We may associate with $B_{1}$ a reduced signature $\mathscr{T}=\left(\begin{array}{ll}\varrho_{1} & \varrho_{4} \\ \varrho_{3} & \varrho_{2}\end{array}\right)$, with $\varrho_{3} \cup \varrho_{4}=\chi$, $\varrho_{1} \cup \varrho_{2}=\Omega-(\chi \cup i)$, such that the four components of $M_{R}-S_{R}$ adjacent to $B_{1}$ have signature $i \mathscr{T}=\left(\begin{array}{ll}\varrho_{1} \cup i & \varrho_{4} \\ \varrho_{3} & \varrho_{2}\end{array}\right), \mathscr{T}^{i}=\left(\begin{array}{ll}\varrho_{1} & \varrho_{4} \cup i \\ \varrho_{3} & \varrho_{2}\end{array}\right)$, etc., obtained by inserting $i$ successively into the sets $\varrho_{1}, \ldots \varrho_{4}$. The van Kampen relations corresponding to $B_{1}$ may be written

$$
\alpha\left({ }^{i} \mathscr{T}\right) \alpha\left({ }_{i} \mathscr{T}\right)=\alpha\left({ }_{i} \mathscr{T}\right) \alpha\left(\mathscr{T}_{i}\right)=\alpha\left(\mathscr{T}_{i}\right) \alpha\left(\mathscr{T}^{i}\right)=\alpha\left(\mathscr{T}^{i}\right) \alpha\left(\mathscr{T}^{i}\right) .
$$

Proof. Take $B \in M(\chi)-M(\chi, 2), B^{\prime} \in M(\chi \cup i)-M(\chi \cup i, 2)$. Then we recall (3.1.2)

$$
\begin{gathered}
l_{j}[x(B, \chi)]=0, \quad j \in \chi \\
\left.\frac{\partial B}{\partial x_{k}}\right|_{x(B, \chi)}+\sum_{j \in \chi} \tau_{j}(B, \chi) \frac{\partial l_{j}}{\partial x_{k}}=0, \quad 1 \leqq k \leqq s+1,
\end{gathered}
$$

and

$$
\begin{gathered}
l_{j}\left[x\left(B^{\prime}, \chi \cup i\right)\right]=0, \quad j \in \chi \cup i \\
\left.\frac{\partial B^{\prime}}{\partial x_{k}}\right|_{x\left(B^{\prime}, \chi \cup i\right)}+\sum_{j \in \chi \cup i} \tau_{j}\left(B^{\prime}, \chi \cup i\right) \frac{\partial l_{j}}{\partial x_{k}}=0, \quad 1 \leqq k \leqq s+1 .
\end{gathered}
$$


Multiplying (3.3.13) by $x_{k}\left(B^{\prime}, \chi \cup i\right)$ and summing over $k$ gives

$$
2 x\left(B^{\prime}, \chi \cup i\right)^{T} B x(B, \chi)+\sum_{j=1}^{r} \tau_{j}(B, \chi) l_{j}\left[x\left(B^{\prime}, \chi \cup i\right)\right]=0,
$$

where we have used $\left.\frac{\partial B}{\partial x_{k}}\right|_{x(B, \chi)}=2 \sum_{j=1}^{s+1} B_{k j} x_{j}(B, \chi)$.

If we manipulate (3.3.15) similarly and subtract the result from (3.3.16), we find

$$
2 x\left(B^{\prime}, \chi \cup i\right)^{T}\left[B-B^{\prime}\right] x(B, \chi)=\tau_{i}\left(B^{\prime}, \chi \cup i\right) l_{i}[x(B, \chi)] .
$$

Here we have used the support properties of $\tau_{j}(B, \psi)$ and $l_{j}[x(B, \psi)]$ in $j$.

In (3.3.17) we set $B=B^{\prime}=B_{1}$ and obtain either $\tau_{i}\left(B_{1}, \chi \cup i\right)=0$ or $l_{i}(x(B, \chi))=0$. In either case it follows from the uniqueness of the null vectors of $B_{1}(\chi), B_{1}(\chi \cup i)$ that these null vectors are the same, and so
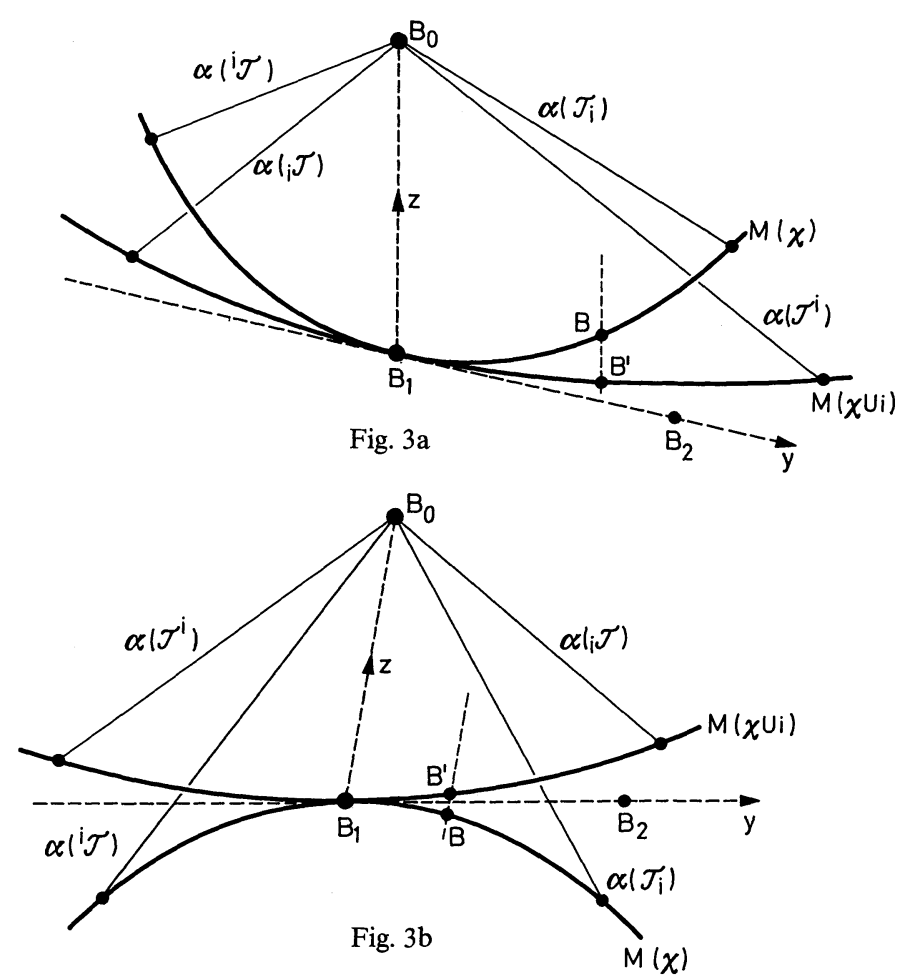

Fig. 3a, b. Sections through a tacnode 
$M(\chi)$ and $M(\chi \cup i)$ are tangent at $B_{1}$. Moreover we then find that both $\tau_{i}\left(B_{1}, \chi \cup i\right)$ and $l_{i}\left(x\left(B_{1}, \chi\right)\right)$ must vanish. From this follows the first part of Lemma 3.3.10 concerning the form of the signatures of the components of $M_{R}-S_{R}$ in the neighbourhood of $B_{1}$, where $\mathscr{T}$ is the signature of any point of $M(\chi)$ or $M(\chi \cup i)$ near $B_{1}$, with the index $i$ removed.

Now consider the plane $B_{0} B_{1} B_{2}$, where $B_{2}$ is a point in the common tangent space to $M(\chi), M(\chi \cup i)$ at $B_{1}$. A point of this plane may be represented parametrically by

$$
B=z B_{0}+(1-z) B_{1}+y B_{2} .
$$

The line defined by varying $z$ in (3.3.18) for fixed $y$ will intersect $M(\chi)$, $M(\chi \cup i)$ in points $z_{1}(y), z_{2}(y)$ in the neighbourhood of $z=0$. In (3.3.17) we set $B=B\left(z_{1}\right), B^{\prime}=B\left(z_{2}\right)$ and obtain

$$
\begin{gathered}
\left(z_{1}-z_{2}\right)\left\{2 x\left(B^{\prime}, \chi \cup i\right)^{T} B_{0} x(B, \chi)-2 x\left(\mathrm{~B}^{\prime}, \chi \cup i\right)^{T} B_{1} x(B, \chi)\right\} \\
=\tau_{i}\left(B^{\prime}, \chi \cup i\right) l_{i}[x(B, \chi)] .
\end{gathered}
$$

Now for $y$ sufficiently small $B$ and $B^{\prime}$ will be very close to $B_{1}$ and the expression in \{ $\}$ in (3.3.19) will be arbitrarily close to $2 x\left(B_{1}, \chi\right)^{T} B_{0} x\left(B_{1}, \chi\right)$, which is positive by the choice of $B_{0}$. We have therefore the two possible configurations illustrated in Fig. 3. In either case the van Kampen relations for the tacnode ( $\$ 2.1$ of [3]) take the form (3.3.11).

We now show that the relations (3.3.11) hold whenever the generators appearing in them are defined.

Lemma 3.3.20. Let $\mathscr{T}=\left(\begin{array}{ll}\eta_{1} & \eta_{4} \\ \eta_{3} & \eta_{2}\end{array}\right)$ with $\eta_{1} \cup \eta_{2} \cup \eta_{3} \cup \eta_{4}=\Omega-i$, and suppose that some consecutive pair in the (cyclicly ordered) sequence ${ }_{\mathfrak{T}} \mathscr{T},{ }_{i} \mathscr{T}, \mathscr{T}_{i}, \mathscr{T}^{i}$ are admissible signatures. Then all four are admissible, and the corresponding generators satisfy (3.3.11).

Proof. The first statement follows directly from the admissibility criterion Lemma 3.3.9. One may then construct a tacnode with reduced signature $\mathscr{T}$ as in the proof of Lemma 3.3.9, and apply Lemma 3.3.10.

We may give

Definition 3.3.21. Let $\mathscr{T}=\left(\begin{array}{ll}\eta_{1} & \eta_{4} \\ \eta_{3} & \eta_{2}\end{array}\right)$, with $\eta_{i} \subset \Omega$ disjoint sets. Then $\mathscr{T}$ is a reduced signature if $r \leqq s$ or if, in the case $r \geqq s+1$, there exist distinct indices $i_{1} \ldots i_{s} \in \Omega$ such that, for $\mathscr{T}_{i_{1} \ldots i_{s}}=\left(\begin{array}{ll}\psi_{1} & \phi \\ \phi & \psi_{2}\end{array}\right)$, we have $\eta_{1} \supset \psi_{1}, \eta_{2} \supset \psi_{2}$ (or $\left.\eta_{1} \supset \psi_{2}, \eta_{2} \supset \psi_{1}\right)$ (see Lemma 3.3.9). If $\mathscr{S}$ is any partition having a reduced partition $\mathscr{T}$ which is a reduced signature, then Lemma 3.3.9 implies that $\mathscr{S}$ is admissible. We may thus define the 
element $\alpha(\mathscr{T}) \in \mathscr{G}(r, s)$ by induction on $\left|\Omega-\left(\eta_{1} \cup \eta_{2} \cup \eta_{3} \cup \eta_{4}\right)\right|$, by

$$
\alpha(\mathscr{T})=\alpha\left(\mathscr{T}^{i}\right) \alpha\left({ }^{i} \mathscr{T}\right) .
$$

Note that from (3.3.11) we have also

$$
\alpha(\mathscr{T})=\alpha\left({ }^{i} \mathscr{T}\right) \alpha\left({ }_{i} \mathscr{T}\right)=\alpha\left({ }_{i} \mathscr{T}\right) \alpha\left(\mathscr{T}_{i}\right)=\alpha\left(\mathscr{T}_{i}\right) \alpha\left(\mathscr{T}^{i}\right) .
$$

We now write down the van Kampen relations corresponding to the transverse intersections of $M(\chi)$ with $M(\psi)$, where $\chi, \psi$ are subsets of $\Omega$ whose symmetric difference contains at least two elements.

Definition 3.3.22. Two partitions $\mathscr{S}_{1}, \mathscr{S}_{2}$ of $\Omega$ are transverse (written $\mathscr{S}_{1} \| \mathscr{S}_{2}$ ) iff for some pair of indices $i j \in \Omega, \mathscr{S}_{1}, \mathscr{S}_{2}$ have reduced partitions $\mathscr{T}_{1}, \mathscr{T}_{2}$ which take one of the following forms:

$$
\begin{array}{ll}
\mathscr{T}_{1}=\left(\begin{array}{ll}
i & j
\end{array}\right), & \mathscr{T}_{2}=\left(\begin{array}{ll}
j & i
\end{array}\right) \\
\mathscr{T}_{1}=\left(\begin{array}{ll}
i \\
j
\end{array}\right), & \mathscr{T}_{2}=\left(\begin{array}{ll}
j & \\
i &
\end{array}\right) ; \\
\mathscr{T}_{1}=\left(\begin{array}{ll}
i j \\
\end{array}\right), & \mathscr{T}_{2}=\left(\begin{array}{ll} 
& j \\
i &
\end{array}\right) ; \\
\mathscr{T}_{1}=\left(\begin{array}{ll}
i j \\
\end{array}\right), & \mathscr{T}_{2}=\left(\begin{array}{ll}
i & \\
& j
\end{array}\right) ;
\end{array}
$$

or a form obtained from any of the above by transposing $\mathscr{T}_{1}$ or $\mathscr{T}_{2}$ or both or by interchanging $\mathscr{T}_{1}$ and $\mathscr{T}_{2}$.

Lemma 3.3.23. If $\mathscr{S}, \mathscr{S}^{\prime}$ are two signatures such that the corresponding sets $\chi=\varrho_{3} \cup \varrho_{4}, \chi^{\prime}=\varrho_{3}^{\prime} \cup \varrho_{4}^{\prime}$ have symmetric difference containing at least two elements, then $M(\chi) \cap M\left(\chi^{\prime}\right) \neq \phi$ iff $\mathscr{S} \| \mathscr{S}^{\prime}$. In that case we have the van Kampen relation

$$
\alpha(\mathscr{S}) \alpha\left(\mathscr{S}^{\prime}\right)=\alpha\left(\mathscr{S}^{\prime}\right) \alpha(\mathscr{S}) .
$$

Proof. Necessity: Let $B \in M(\mathscr{S}) \cap M\left(\mathscr{S}^{\prime}\right)$. By repeating the manipulations used to derive (3.3.17) we obtain the identity

$$
\sum_{k \in \chi-\chi^{\prime}} \tau_{k}(B, \chi) l_{k}\left(x\left(B, \chi^{\prime}\right)\right)-\sum_{k \in \chi^{\prime}-\chi} \tau_{k}\left(B, \chi^{\prime}\right) l_{k}(x(B, \chi))=0,
$$

where we have used the support properties of $\tau_{j}(B, \psi), l_{j}(x(B, \psi))$ in $j$ to restrict the summations to indices $k$ which give non-zero terms. At least two terms in (3.3.24) must have opposite signs, say those given by $k=i, j$. The corresponding restriction on $\mathscr{S}, \mathscr{S}^{\prime}$ is precisely $\mathscr{S} \| \mathscr{S}^{\prime}$. 
Sufficiency: Let $\mathscr{S}, \mathscr{S}^{\prime}$ be two signatures with $\mathscr{S} \| \mathscr{S}^{\prime}$. Since $\mathscr{S}, \mathscr{S}^{\prime}$ are admissible there exist $x, x^{\prime} \in \mathbb{P}^{s}$ such that

$$
\begin{aligned}
& l_{j}(x)=0 \text { for } j \in \chi, \\
& l_{j}(x)>0 \text { for } j \in \varrho_{1}, \quad l_{j}(x)<0 \text { for } j \in \varrho_{2},
\end{aligned}
$$

and similarly for $x^{\prime}$. Since $\mathscr{S} \| \mathscr{S}^{\prime}$ we may choose $\tau_{j}$, $\tau_{j}^{\prime}$ such that

$$
\begin{aligned}
& \tau_{j}=0 \text { for } j \notin \chi, \quad \tau_{j}>0 \text { for } j \in \varrho_{3}, \quad \tau_{j}<0 \text { for } j \in \varrho_{4}, \\
& \tau_{j}^{\prime}=0 \text { for } j \notin \chi^{\prime}, \quad \tau_{j}^{\prime}>0 \text { for } j \in \varrho_{3}^{\prime}, \quad \tau_{j}^{\prime}<0 \text { for } j \in \varrho_{4}^{\prime} \text {, }
\end{aligned}
$$

and

$$
\sum_{k \in \chi-\chi^{\prime}} \tau_{k} l_{k}\left(x^{\prime}\right)-\sum_{k \in \chi^{\prime}-\chi} \tau_{k}^{\prime} l_{k}(x)=0 .
$$

We must now construct $B$ so that $x=x(B, \chi), x^{\prime}=x\left(B, \chi^{\prime}\right)$ and

$$
\left.\frac{\partial B}{\partial x_{k}}\right|_{x}+\sum_{j} \tau_{j} \frac{\partial l_{j}}{\partial x_{k}}=0
$$

and similarly for $x^{\prime}$. We may regard $B$ as a linear map $B: \mathbb{C}^{s+1} \rightarrow \mathbb{C}^{s+1 *}$ (the dual vector space). Then the above conditions on $B$ may be rewritten in the form:

$$
\text { 1. } \begin{aligned}
B x & =-\sum_{j} \tau_{j} l_{j} \in \mathbb{C}^{s+1 *}, \\
B x^{\prime} & =-\sum_{j} \tau_{j}^{\prime} l_{j} \in \mathbb{C}^{s+1 *} .
\end{aligned}
$$

In addition we require

2. $B$ is symmetric, i.e. $\forall y, y^{\prime} \in \mathbb{C}^{s+1}$

$$
B y\left(y^{\prime}\right)=B y^{\prime}(y) \text {. }
$$

We may find a map $B$ satisfying the linear conditions 1 and 2 iff the compatibility condition $B x\left(x^{\prime}\right)=B x^{\prime}(x)$ is satisfied. But this is precisely (3.3.25).

\subsection{Algebraic Properties of the Group}

Because we do not prove that the relations we have obtained exhaust those of the fundamental group, it is convenient to make

Definition 3.4.1. Let $l_{1}, \ldots l_{r}$ be a set of real linear forms on $\mathbb{C}^{s+1}$, and let $\mathscr{A}$ be the corresponding set of signatures admissible under Lemma 3.3.9. Then define $\mathscr{H}(r, s, \mathscr{A})$ to be the group with generators $\alpha(\mathscr{S}), \mathscr{S} \in \mathscr{A}$, and relations

$$
\alpha\left(\mathscr{T}^{i}\right) \alpha\left({ }^{i} \mathscr{T}\right)=\alpha\left({ }^{i} \mathscr{T}\right) \alpha\left({ }_{i} \mathscr{T}\right)=\alpha\left({ }_{i} \mathscr{T}\right) \alpha\left(\mathscr{T}_{i}\right)=\alpha\left(\mathscr{T}_{i}\right) \alpha\left(\mathscr{T}^{i}\right),
$$


whenever $\mathscr{T}^{i},{ }^{i} \mathscr{T} \in \mathscr{A}$, and

$$
\alpha(\mathscr{S}) \alpha\left(\mathscr{S}^{\prime}\right)=\alpha\left(\mathscr{S}^{\prime}\right) \alpha(\mathscr{S})
$$

whenever $\mathscr{S} \| \mathscr{S}^{\prime}$.

Note that we have not included any label such as $\mathscr{A}$ in our notation $\mathscr{G}(r, s)$ for the fundamental group. This is due to Lemma 3.4.2, whose (geometric) proof we omit.

Lemma 3.4.2. The fundamental group of the parameter space of the integral (2.5) is independent of the choice of $l_{j}$ satisfying the basic linear independence requirement.

We have stated Lemma 3.4.2 only for completeness, and will not use it further. We believe, however, that $\mathscr{H}(r, s, \mathscr{A}) \simeq \mathscr{G}(r, s)$, and for this reason will usually suppress the dependence of $\mathscr{H}(r, s)$ on $\mathscr{A}$. Note that in any case $\S 3.3$ implies that there exists an onto map $\phi: \mathscr{H}(r, s, \mathscr{A})$ $\rightarrow \mathscr{G}(r, s)$.

We now give a series of disconnected remarks about $\mathscr{H}(r, s)$.

Remark 3.4.3. For any $s^{\prime} \geqq r, \mathscr{H}\left(r, s^{\prime}\right)=\mathscr{H}(r, r) \equiv \mathscr{H}(r)$. This follows from Lemma 3.3.9, since for $s^{\prime} \geqq r$ there are no restrictions on the signatures.

Remark 3.4.4. We give some examples of positional characteristics and the restrictions they impose upon the signatures which will be relevant for applications.

A. $r=s+1, l_{j}(x)=x_{j}, j=1, \ldots r$. In this case any admissible signature must have as reduced signature $\mathscr{T}_{1} \ldots \hat{i} \ldots r$ for some $i$. The resulting condition on $\mathscr{S}=\left(\begin{array}{ll}\varrho_{1} & \varrho_{4} \\ \varrho_{3} & \varrho_{2}\end{array}\right)$ is that $\varrho_{1} \cup \varrho_{2} \neq \phi$.

B. $r=s+2 ; l_{j}(x)=x_{j}, j=1, \ldots s+1 ; l_{r}(x)=-\sum_{i=1}^{s+1} x_{i}^{\prime}$. In this case one calculates that $\mathscr{T}_{\Omega-\{i, j\}}=\left(\begin{array}{ll}i & \phi \\ \phi & j\end{array}\right)$, so that any $\mathscr{S}=\left(\begin{array}{ll}\varrho_{1} & \varrho_{4} \\ \varrho_{3} & \varrho_{2}\end{array}\right)$ must have $\varrho_{1} \neq \phi, \varrho_{2} \neq \phi$.

C. For any $r \geqq s+1$ we obtain an easily computed positional characteristic by choosing

$$
l_{j}(x)=\sum_{i=1}^{s+1} x_{i} \xi_{j}^{k(i)}
$$

where $0<\xi_{1} \ll \xi_{2} \ll \cdots \ll \xi_{r}$, and the integers $k(i)$ satisfy $k(i)<k(i+1)$. Then the positional characteristic is easily computed: $\left(i_{1} \ldots i_{s+1}\right)$ is positive for $i_{1}<i_{2}<\cdots<i_{s+1}$ and is anti-symmetric in its arguments. 
From this we find that if $|\chi|=s$, the corresponding reduced signature $\mathscr{T}_{\chi}=\left(\begin{array}{ll}\varrho_{1} & \phi \\ \phi & \varrho_{2}\end{array}\right)$ is given by

$$
\begin{aligned}
& \varrho_{1}=\{j \notin \chi|| \chi \cap(j, r] \mid \text { is even }\}, \\
& \varrho_{2}=\{j \notin \chi|| \chi \cap(j, r] \mid \text { is odd }\} .
\end{aligned}
$$

$($ Here $(j, r]=\{j+1, \ldots r\}$.)

Remark 3.4.5. The reduced signatures introduced in Def. 3.3.21 satisfy relations similar to those of the signatures themselves. Take $\mathscr{T}=\left(\begin{array}{ll}\varrho_{1} & \varrho_{4} \\ \varrho_{3} & \varrho_{2}\end{array}\right)$, $\mathscr{T}^{\prime}=\left(\begin{array}{ll}\varrho_{1}^{\prime} & \varrho_{4}^{\prime} \\ \varrho_{3}^{\prime} & \varrho_{2}^{\prime}\end{array}\right)$ to be reduced signatures. $\mathscr{T}$ and $\mathscr{T}^{\prime}$ are defined to be transverse $\left(\mathscr{T} \| \mathscr{T}^{\prime}\right)$ just as in Def. 3.3.22, i.e., whenever they contain the appropriate reduced signatures. Then

A. $\mathscr{T} \| \mathscr{T}^{\prime}$ implies $\alpha(\mathscr{T}) \alpha\left(\mathscr{T}^{\prime}\right)=\alpha\left(\mathscr{T}^{\prime}\right) \alpha(\mathscr{T})$.

B. Suppose $\varrho_{i}^{\prime}=\varrho_{i} \cup \eta_{i}$, for all $i=1, \ldots 4$. Then

where

$$
\alpha(\mathscr{T}) \alpha\left(\mathscr{T}^{\prime}\right)=\alpha\left(\mathscr{T}^{\prime \prime}\right) \alpha(\mathscr{T}),
$$

$$
\mathscr{T}^{\prime \prime}=\left(\begin{array}{ll}
\varrho_{1} \cup \eta_{2} & \varrho_{4} \cup \eta_{3} \\
\varrho_{3} \cup \eta_{4} & \varrho_{2} \cup \eta_{1}
\end{array}\right)
$$

C. Suppose $\varrho_{i}^{\prime} \supset \varrho_{i}, i=1, \ldots 4$, and that $k \in \Omega, k \notin \varrho_{1}^{\prime} \cup \varrho_{2}^{\prime} \cup \varrho_{3}^{\prime} \cup \varrho_{4}^{\prime}$. Then

$$
\alpha\left(\mathscr{T}^{k}\right) \alpha\left({ }^{k} \mathscr{T}^{\prime}\right)=\alpha\left(\mathscr{T}_{k}^{\prime}\right) \alpha\left(\mathscr{T}^{k}\right),
$$

together with three similar equations obtained from (3.4.7) by rotating the index $k$ in all signatures. Note that in each of these four equations the index $k$ moves on $\mathscr{T}^{\prime}$, but not on $\mathscr{T}$.

These relations may be easily proved by induction on the number of indices which do not appear in $\mathscr{T}$ and $\mathscr{T}^{\prime}$; we omit proofs.

Remark 3.4.8. Properties of $\mathscr{H}(r)$ :

A. There is a natural homomorphism $i: \mathscr{H}(r, s) \rightarrow \mathscr{H}(r)$ defined by mapping $\alpha(\mathscr{S}) \in \mathscr{H}(r, s)$ into $\alpha(\mathscr{S}) \in \mathscr{H}(r)$. We do not know if this is an injection but we will show that the monodromy representation of $\mathscr{H}(r, s)$ factors through this homomorphism and is obtained as a subrepresentation of the monodromy representation of $\mathscr{H}(r)$.

B. The elements $\alpha(\mathscr{S}) \in \mathscr{H}(r)$ defined by signatures $\mathscr{S}$ having a common reduced signature $\mathscr{T} \neq\left(\begin{array}{ll}\phi & \phi \\ \phi & \phi\end{array}\right)$ generate a subgroup $\mathscr{K}(u)$ whose structure depends only on the number $u$ of indices in $\Omega-\mathscr{T}$. $\mathscr{K}(u)$ is defined by the same generators and relations as $\mathscr{H}(u)$ except that in $\mathscr{K}(u)$ we do not have $\alpha(\mathscr{S})=\alpha\left(\mathscr{S}^{T}\right)$. 
C. The elements $\alpha(\mathscr{T}) \in \mathscr{H}(r)$, with $\mathscr{T}$ a reduced signature not containing indices in a given subset $\Lambda$ of $\Omega$, generate a group which is isomorphic with $\mathscr{H}(r-|\Lambda|)$.

D. From (3.4.6), (3.4.7) we find that conjugation of $\alpha(\mathscr{S})$ by $\alpha\left(\begin{array}{ll}i & \phi \\ \phi & \phi\end{array}\right)$ gives, for any signature $\mathscr{S}$ and index $i$, the element $\alpha\left(\mathscr{S}^{\prime}\right)$; where $\mathscr{S}^{\prime}$ is obtained from $\mathscr{S}$ by transposing the position of the index $i$. It follows that the elements $\beta_{i}=\left[\alpha\left(\begin{array}{ll}i & \phi \\ \phi & \phi\end{array}\right)\right]^{2}$ lie in the center of $\mathscr{H}(r)$. Similarly, the elements $\beta_{i}^{\prime}=\left[\alpha\left(\begin{array}{ll}\phi & i \\ \phi & \phi\end{array}\right)\right]^{2}$ and $\beta=\alpha\left(\begin{array}{ll}\phi & \phi \\ \phi & \phi\end{array}\right)$ lie in the center. The elements $\alpha\left(\begin{array}{ll}i & \phi \\ \phi & \phi\end{array}\right), 1 \leqq i \leqq r$, generate an abelian subgroup. It turns out that these form a "complete set of commuting observables" for the monodromy representation, i.e. their simultaneous eigenvectors form a basis in the representation space and are uniquely characterized by their eigenvalues. However, we do not make use of this basis in $\S 4$ as it is not convenient for the physical applications of $\S 5$.

Remark 3.4.9. For a deeper understanding of the group $\mathscr{H}(r)$ it would be desirable to have a solution of the word problem for $\mathscr{H}(r)$. While the word problem is known to be unsolvable for many finitely presented groups, it is reasonable to suppose that it will be solvable for finitely presented groups which arise in a natural geometrical context, such as $\mathscr{H}(r)$. This conjecture is supported by a comparison of the structure of $\mathscr{H}(r)$ with the structure of the braid group and other related groups for which the word problem has been solved [19]. In fact the method of [19] seems well adapted to attack the word problem for $\mathscr{H}(r)$ - with $\beta$ playing the role of $\Delta^{2}$ in [19].

\section{§4. The Representation Theory}

\subsection{Introductory Remarks}

Let $V$ be the vector space spanned by the germs of the function (2.5) at the base point $B_{0}$. In this section we determine the representation $\mathscr{L}$ of the group ring of the fundamental group acting on $V$ :

$$
\mathscr{L}: \mathbb{C}(\mathscr{G}(r, s)) \rightarrow L(V) .
$$

(We suppress the dependence of $\mathscr{L}$ on $r, s, \mu, \lambda_{1} \ldots \lambda_{r}$, and $l_{1} \ldots l_{r}$. However, we suppose throughout this section that the $\mu, \lambda_{1}, \ldots, \lambda_{r}$ are generic; we will show that $\mathscr{L}$ is independent of the choice of integration cycle $\gamma$.) 
By composing the map $\mathscr{L}$ with the homomorphism $\phi: \mathbb{C}(\mathscr{H}(r, s))$ $\rightarrow \mathbb{C}(\mathscr{G}(r, s))$ introduced in 3.4 , we obtain a representation $\mathscr{L}^{\prime}=\mathscr{L} \phi$ of $\mathbb{C}(\mathscr{H}(r, s))$. Our determination of $\mathscr{L}^{\prime}$ will be carried out in several stages:

1 . We determine the conditions on the representations which arise from a local analysis in the integration space, and from the homogeneity of (2.5) in the variables $B_{i j}$. To determine these conditions we need the fact that there exists an integration cycle $\gamma_{0}$ such that the corresponding function (2.5) is singular (on some sheet) on each of the components $M(\chi)$ of $M$. This will be established in Appendix A. Initially we will take $\gamma=\gamma_{0}$. However, when we have determined that the representation corresponding to this choice of integration cycle is irreducible we will be able to show that any other choice of $\gamma$ which does not give a vanishing integral defines the same function and hence the same monodromy representation $\mathscr{L}$.

2. We show that these conditions determine uniquely the trace of the representation $\mathscr{L}^{\prime}$.

3. We construct a representation of $\mathbb{C}(\mathscr{H}(r, s))$ which has the correct trace, and show that it is irreducible. is $\mathscr{L}^{\prime}$.

4. We then apply Lemma 4.1.1 to show that this representation

Lemma 4.1.1. If $\mathscr{G}$ is any group and $\mathscr{L}_{1}, \mathscr{L}_{2}$ are finite-dimensional representations of $\mathscr{G}$ on $V_{1}, V_{2}$ such that

a) For any $x \in \mathbb{C}(\mathscr{G}), \operatorname{tr}\left(\mathscr{L}_{1}(x)\right)=\operatorname{tr}\left(\mathscr{L}_{2}(x)\right)$;

b) $\mathscr{L}_{1}$ is irreducible;

then $\mathscr{L}_{1}$ and $\mathscr{L}_{2}$ are equivalent.

Proof. This is a slight extension of a well-known theorem [9, p. 174].

Theorem 4.1.2. Any two completely reducible representations of a finite-dimensional algebra, having the same trace, are equivalent.

To apply Theorem 4.1.2, consider the representation $\mathscr{L}_{1} \oplus \mathscr{L}_{2}: \mathbb{C}(\mathscr{G})$ $\rightarrow L\left(V_{1} \oplus V_{2}\right)$. Let $R=\left(\mathscr{L}_{1} \oplus \mathscr{L}_{2}\right)(\mathbb{C}(\mathscr{G})) ; R$ is a finite-dimensional algebra. We define by projection representations $\mathscr{L}_{1}^{\prime}, \mathscr{L}_{2}^{\prime}$ of $R$, having the same trace. $\mathscr{L}_{1}^{\prime}$ is again irreducible. Let $\{0\}=W_{0} \subset W_{1} \subset \cdots \subset W_{k}=V_{2}$ be a maximal sequence of subspaces of $V_{2}$ invariant under $\mathscr{L}_{2}^{\prime}$; then the induced representations $\mathscr{L}_{2}^{i)}$ of $R$ on $W_{i} / W_{i-1}$ are irreducible, and $\mathscr{L}_{2}^{\prime \prime}=\sum_{i=1}^{k} \oplus \mathscr{L}_{2}^{i)}$ is a completely reducible representation of $R$ with the same trace as $\mathscr{L}_{2}^{\prime}$ or $\mathscr{L}_{1}^{\prime}$. Thus Theorem 4.1.2 implies that $\mathscr{L}_{2}^{\prime \prime}$ is equivalent to $\mathscr{L}_{1}^{\prime}$. But $\mathscr{L}_{1}^{\prime}$ is irreducible, hence $\mathscr{L}_{2}^{\prime \prime}$ is irreducible and so $\mathscr{L}_{2}^{\prime}=\mathscr{L}_{2}^{\prime \prime}$, and $\mathscr{L}_{1}^{\prime}$ and $\mathscr{L}_{2}^{\prime}$ are equivalent. This implies that $\mathscr{L}_{1}$ and $\mathscr{L}_{2}$ are equivalent. 
5. The representation of stage 3 will be constructed first for $\mathbb{C}(\mathscr{H}(r))$. Then the representation of $\mathbb{C}(\mathscr{H}(r, s))$ will be obtained as a subrepresentation of the representation of $\mathbb{C}(\mathscr{H}(r))$ (cf. Remark 3.4.8 (A)).

\subsection{The Conditions on the Representation}

(i) Consequences of the Picard-Lefschetz theorem.

Let $\mathscr{S}$ be a signature, $\chi=\varrho_{3} \cup \varrho_{4}$. We denote by $X(\chi)$ the constant

$$
X(\chi)=(-1)^{s-|x|} \exp \left\{2 \pi i\left(-\mu+\sum_{j \in \chi} \lambda_{j}\right)\right\}
$$

and by $a(\mathscr{S}) \in \mathbb{C}(\mathscr{G}(r, s))$ the discontinuity operator

$$
a(\mathscr{S})=\alpha(\mathscr{S})-1
$$

Then

$$
\left[\mathscr{L}^{\prime}(a(\mathscr{S}))\right]^{2}=(X(\chi)-1)\left[\mathscr{L}^{\prime}(a(\mathscr{S}))\right] ;
$$

furthermore $\mathscr{L}^{\prime}(a(\mathscr{S}))[X(\chi)-1]^{-1}$ is a one-dimensional projection operator. We introduce the vector space $V^{*}$ dual to $V$, together with the bilinear pairing $(a, b), a \in V^{*}, b \in V$. Thus

$$
\mathscr{L}^{\prime}(a(\mathscr{S}))=u(\mathscr{S}) \otimes v(\mathscr{S})
$$

where $u(\mathscr{S}) \in V$ and $v(\mathscr{S}) \in V^{*}$. (4.2:1) implies $(v(\mathscr{S}), u(\mathscr{S}))=X(\chi)-1$.

ii) Localization conditions.

If two signatures $\mathscr{S}_{1}$ and $\mathscr{S}_{2}$ are transverse (Definition 3.3.22),

$$
\mathscr{L}^{\prime}\left(a\left(\mathscr{S}_{1}\right)\right) \mathscr{L}^{\prime}\left(a\left(\mathscr{S}_{2}\right)\right)=0
$$

iii) Effective intersections.

The condition satisfied by the integration cycle $\gamma_{0}$ (Remark 1 of 4.1) implies that for any signature $\mathscr{S}, \mathscr{L}^{\prime}(\alpha(\mathscr{S})) \neq 1$. From the analysis of an effective intersection (given in the case in which the $\lambda_{j}, \mu$ are integers in [10] and [11]) we find that for any two signatures $\mathscr{S}_{1}, \mathscr{S}_{2}$ differing only in the position of a single index, corresponding to components $M\left(\chi_{1}\right), M\left(\chi_{2}\right)$ of $M$ with a common tangent,

$$
\mathscr{L}^{\prime}\left(a\left(\mathscr{S}_{1}\right) a\left(\mathscr{S}_{2}\right)-a\left(\mathscr{S}_{2}\right) a\left(\mathscr{S}_{1}\right)\right) \neq 0 .
$$

According to $(i)$ there exists a constant $C\left(\mathscr{S}_{1}, \mathscr{S}_{2}\right)$ such that

$$
a\left(\mathscr{S}_{1}\right) a\left(\mathscr{S}_{2}\right) a\left(\mathscr{S}_{1}\right)=C\left(\mathscr{S}_{1}, \mathscr{S}_{2}\right) a\left(\mathscr{S}_{1}\right) .
$$

The bicommutation relation

$$
\left[\alpha\left(\mathscr{S}_{1}\right) \alpha\left(\mathscr{S}_{2}\right)\right]^{2}=\left[\alpha\left(\mathscr{S}_{2}\right) \alpha\left(\mathscr{S}_{1}\right)\right]^{2}
$$


which follows from (3.3.11) (see also [1] Table 1) gives

$$
\left[C\left(\mathscr{S}_{1}, \mathscr{S}_{2}\right)+X\left(\chi_{1}\right)+X\left(\chi_{2}\right)\right] \mathscr{L}^{\prime}\left(a\left(\mathscr{S}_{1}\right) a\left(\mathscr{S}_{2}\right)-a\left(\mathscr{S}_{2}\right) a\left(\mathscr{S}_{1}\right)\right)=0,
$$

and so by (4.2.4)

$$
C\left(\mathscr{S}_{1}, \mathscr{S}_{2}\right)=-\left\{X\left(\chi_{1}\right)+X\left(\chi_{2}\right)\right\} .
$$

(iv) Homogeneity.

The word at infinity $w_{\infty}$ (cf. [1]) is represented by a multiple of the unit matrix,

$$
\mathscr{L}^{\prime}\left(w_{\infty}\right)=\exp (2 \pi i \mu) 1=c_{\infty} 1 .
$$

We will not need to determine $w_{\infty}$ explicitly as a product of the generators $\alpha(\mathscr{S})$ of $\mathscr{G}(r, s)$ (though in Appendix $B$ we show how this may be done in the particular case $r=s+1)$. It will be sufficient to note that $w_{\infty}^{-1}$ may be expressed as a product of generators $\alpha(\mathscr{S})$ such that the number of generators in the product corresponding to a given variety $M(\chi)$ is equal to the degree $s+1-|\chi|$ of $M(\chi)$; such an expression is obtained by writing down $w_{\infty}$ in any generic line through $B_{0}$.

Definition 4.2.9. The ${ }^{*}$-involution in $\mathbb{C}(\mathscr{G}(r, s))$ is given by

for all $c_{i} \in \mathbb{C}, g_{i} \in \mathscr{G}(r, s)$.

$$
\left(\sum c_{i} g_{i}\right)^{*}=\sum \bar{c}_{i} g_{i}^{-1}
$$

Remark 4.2.10. If the parameters $\lambda_{i}, \mu$ are real the conditions (i)-(iv) on $\mathscr{L}$ are *-invariant. Since $\mathscr{L}$ proves to be uniquely characterized by these conditions, it follows that the *-operation may be defined also on the representation matrices.

\subsection{Formulae for $\mathscr{H}(r)$}

In this section we derive certain algebraic formulae which hold in the representation $\mathscr{L}^{\prime}$ of $\mathbb{C}(\mathscr{H}(r))$. These will be used in the calculation of traces for general $r$ and $s(4.4)$ and in the construction of the representation $\mathscr{L}^{\prime}(4.5)$.

We first introduce a minimal set of generators of $\mathscr{H}(r)$. Let $\mathscr{T}=\left(\begin{array}{ll}\varrho_{1} & \varrho_{4} \\ \varrho_{3} & \varrho_{2}\end{array}\right)$ be a fixed signature. For any $\chi \subset \Omega$, let $\mathscr{S}(\chi)=\left(\begin{array}{ll}\varrho_{1}^{\prime} & \varrho_{4}^{\prime} \\ \varrho_{3}^{\prime} & \varrho_{2}^{\prime}\end{array}\right)$ be the signature obtained from $\mathscr{T}$ by rotating all indices $j \in \chi$ clockwise, i.e.

$$
\begin{aligned}
\varrho_{i} \cap(\Omega-\chi) & =\varrho_{i}^{\prime} \cap(\Omega-\chi), & & (i=1, \ldots 4) \\
\varrho_{4}^{\prime} \cap \chi & =\varrho_{1} \cap \chi & & \text { etc. }
\end{aligned}
$$


and let $\alpha(\chi)=\alpha(\mathscr{S}(\chi)) ; a(\chi)=\alpha(\chi)-1$. The $\alpha(\chi)$ generate $\mathscr{H}(r)$, as may be seen using (3.3.11), and are minimal in the sense that they include one generator for each irreducible component of $M$.

In the remainder of this section we will take $\mathscr{T}=\left(\begin{array}{ll}\Omega & \phi \\ \phi & \phi\end{array}\right)$, so that $\mathscr{S}(\chi)=\left(\begin{array}{ll}\Omega-\chi & \chi \\ \phi & \phi\end{array}\right)$. The formulae we derive depend on this choice. But very similar formulae hold for any choice of $\mathscr{T}$; in particular, the important Remark 4.3.7 holds for any $\mathscr{T}$.

Lemma 4.3.1. For any $\chi_{1}, \chi_{2} \subset \Omega, \mathscr{L}^{\prime}\left(a\left(\chi_{1}\right) a\left(\chi_{2}\right)\right)=0$ unless $\chi_{1} \subset \chi_{2}$ or $\chi_{2} \subset \chi_{1}$.

Proof. From (4.2.3).

Lemma 4.3.2. Suppose $\chi_{1} \subsetneq \chi_{2} \subsetneq \chi_{3}$. Then

$$
\begin{aligned}
& \mathscr{L}^{\prime}\left\{a\left(\chi_{3}\right) a\left(\chi_{2}\right) a\left(\chi_{1}\right)\right\}=-\mathscr{L}^{\prime}\left\{a\left(\chi_{3}\right) a\left(\chi_{1}\right)\right\}, \\
& \mathscr{L}^{\prime}\left\{a\left(\chi_{1}\right) a\left(\chi_{2}\right) a\left(\chi_{3}\right)\right\}=X\left(\chi_{2}\right) \mathscr{L}^{\prime}\left\{a\left(\chi_{1}\right) a\left(\chi_{3}\right)\right\} .
\end{aligned}
$$

Proof. Suppose first that $\chi_{2}=\chi_{1} \cup\{k\}$, for some $k \in \Omega$. Let $\mathscr{S}=\left(\begin{array}{cc}\Omega-\chi_{1}-k & \chi_{1} \\ \phi & k\end{array}\right)$. Then from (3.3.11)

$$
a(\mathscr{S})=\alpha\left(\chi_{2}\right) a\left(\chi_{1}\right) \alpha\left(\chi_{2}\right)^{-1},
$$

and from (4.2.3)

$$
\mathscr{L}^{\prime}\left\{a\left(\chi_{3}\right) a(\mathscr{S})\right\}=0,
$$

Thus

$$
\mathscr{L}^{\prime}\left\{a\left(\chi_{3}\right) \alpha\left(\chi_{2}\right) a\left(\chi_{1}\right)\right\}=0,
$$

and this implies (4.3.3). (4.3.3) for general $\chi_{2}$ follows by repeated application of this special case. (4.3.4) may be shown similarly; we also remark that (4.3.4) is the * of (4.3.3) (see Definition 4.2.9).

Lemma 4.3.5. Suppose $\chi_{1} \subsetneq \chi_{2}$. Then

$$
\begin{aligned}
& \mathscr{L}^{\prime}\left\{a\left(\chi_{1}\right) a\left(\chi_{2}\right) a\left(\chi_{1}\right)\right\}=C\left(\chi_{1}, \chi_{2}\right) \mathscr{L}^{\prime}\left\{a\left(\chi_{1}\right)\right\} \\
& \mathscr{L}^{\prime}\left\{a\left(\chi_{2}\right) a\left(\chi_{1}\right) a\left(\chi_{2}\right)\right\}=C\left(\chi_{2}, \chi_{1}\right) \mathscr{L}^{\prime}\left\{a\left(\chi_{2}\right)\right\}
\end{aligned}
$$

with

where

$$
C\left(\chi_{1}, \chi_{2}\right)=C\left(\chi_{2}, \chi_{1}\right)=-X\left(\chi_{1}\right) \prod_{i \in\left(\chi_{2}-\chi_{1}\right)}\left\{1+Y_{i}\right\},
$$

$$
Y_{i}=-e^{2 \pi i \lambda_{t}}
$$


Proof. If $\left|\chi_{2}-\chi_{1}\right|=1$, this is simply (4.2.5). It follows for general $\chi_{1}, \chi_{2}$ by introducing sets $\chi_{1}=\psi_{0} \subset \psi_{1} \ldots \subset \psi_{k}=\chi_{2}$, with $k=\left|\chi_{2}-\chi_{1}\right|$ and $\left|\psi_{i}-\psi_{i-1}\right|=1$, writing

$$
\mathscr{L}^{\prime}\left\{a\left(\chi_{1}\right) a\left(\chi_{2}\right) a\left(\chi_{1}\right)\right\}=\prod_{i=1}^{k-1}\left\{-X\left(\psi_{k}\right)\right\}^{-1} \mathscr{L}^{\prime}\left\{a\left(\psi_{0}\right) \ldots a\left(\psi_{k}\right) \ldots a\left(\psi_{0}\right)\right\},
$$

(see Lemma 4.3.2), and applying (4.2.5) repeatedly.

Lemma 4.3.6. Suppose $\chi_{1} \leqq \chi_{2} \leqq \chi_{3}$. Then for $i \neq j \neq k \in\{1,2,3\}$, we have $\mathscr{L}^{\prime}\left\{a\left(\chi_{i}\right) a\left(\chi_{j}\right) a\left(\chi_{k}\right)\right\}=C_{i j k} \mathscr{L}^{\prime}\left\{a\left(\chi_{i}\right) a\left(\chi_{k}\right)\right\}$, with

$$
\begin{aligned}
& C_{123}=X\left(\chi_{2}\right), \\
& C_{321}=-1, \\
& C_{231}=X\left(\chi_{2}\right) \prod_{\chi_{3}-\chi_{2}}\left(1+Y_{i}\right), \\
& C_{312}=X\left(\chi_{1}\right) \prod_{\chi_{2}-\chi_{1}}\left(1+Y_{i}\right), \\
& C_{213}=-\prod_{\chi_{2}-\chi_{1}}\left[1+Y_{i}^{-1}\right], \\
& C_{132}=-\prod_{\chi_{3}-\chi_{2}}\left(1+Y_{i}\right) .
\end{aligned}
$$

Proof. Follows directly from (4.3.2) and (4.3.5).

Remark 4.3.7. Lemma 4.3.6 enables one to reduce any product of the elements $\mathscr{L}^{\prime}\{a(\chi)\}$ to a multiple of a product of the form $\mathscr{L}^{\prime}\left\{a\left(\chi_{3}\right) a(\Omega) a\left(\chi_{2}\right)\right\}$. Note that we have not used the formula $a(\mathscr{S})$ $=a\left(\mathscr{S}^{T}\right)$ at all in this section. Thus Lemmas 4.2.1-4.2.6 hold also for any representation of $\mathscr{K}(r)$ (Remark 3.4.8 $(B)$ ) which satisfies the conditions of 4.2 .

\subsection{Determination of the Trace of the Representation $\mathscr{L}^{\prime}$}

We first reduce the problem of computing the trace of $\mathscr{L}^{\prime}$ to the problem of computing the traces of products of discontinuity operators $\mathscr{L}^{\prime}(a(\mathscr{S}))$. To do this we may use the homogeneity condition (4.2.8). If in (4.2.8) we take the trace of both sides we obtain an expression for the trace of the unit element (i.e. the dimension $d(r, s)$ of the representation) as a sum of traces of products of the $\mathscr{L}^{\prime}(a(\mathscr{S}))$. However, for general $r, s$ we do not have an explicit expression for $w_{\infty}$ so we prefer to use (4.2.8) in a different way (taken from [12]) which yields immediately a formula for $d(r, s)$. We take the determinant of both sides of (4.2.8) and use the remark following (4.2.8) on the structure of $w_{\infty}$, together with 
the relation $\operatorname{det} \mathscr{L}^{\prime}(\alpha(\mathscr{S}))=X(\chi)$. This gives

$$
\prod_{\substack{\phi \subset \chi \subset \Omega \\|\chi| \leqq s}} X(\chi)^{s+1-|\chi|}=c_{\infty}^{-d(r, s)} .
$$

From (4.2.0) we can write the left-hand side of (4.4.1) in the form $c_{\infty}^{-d}$ where

$$
\begin{aligned}
d & =\sum_{j=0}^{s}\left(\begin{array}{l}
r \\
j
\end{array}\right)(s+1-j)-2 \sum_{j=1}^{s}\left(\begin{array}{c}
r-1 \\
j-1
\end{array}\right)(s+1-j) \\
& =\sum_{k=0}^{s}\left(\begin{array}{c}
r-1 \\
k
\end{array}\right)
\end{aligned}
$$

We have thus proved

Lemma 4.4.3. The representation $\mathscr{L}^{\prime}$ has dimension $d(r, s)$ given by (4.4.2).

Next we use (4.2.1) to calculate the traces of the individual $\mathscr{L}^{\prime}(a(\mathscr{S}))$.

Lemma 4.4.4. $\quad \operatorname{tr} \mathscr{L}^{\prime}(a(\mathscr{S}))=X(\chi)-1$.

To proceed further we require a minimal set of generators for $\mathscr{H}(r, s)$. We obtain such a set by taking the generators corresponding to the intersections with $M$ of a certain.non-generic line through the base point $B_{0}$. Note, however, that the following geometrical constructions serve only to motivate and organize the combinatorics. Essentially we need only the defining relations on the generators $\alpha(\mathscr{S})$ of $\mathscr{H}(r, s)$. Let $Y$ be a point in the integration space $\mathbb{P}^{s}$ not lying on any $l_{i}$, and choose coordinates $y_{j}, 1 \leqq j \leqq s+1$, such that $Y=(0, \ldots, 0,1)$. The quadratic forms

$$
B(R)=\left(y_{1}^{2}+\cdots+y_{s}^{2}\right)-R y_{s+1}^{2}
$$

define a line in the parameter space $W$. For $R<0, B(R)$ is positive definite so we may suppose our base point $B_{0}$ is given by some negative value $R_{0}$ of $R$. The line intersects each component $M(\chi)$ of $M$ in just one point, given by $R=R(\chi)$ say. We may choose $Y$ so that the numbers $R(\chi)$ are all distinct. Let $0=R_{1}<R_{2}<R_{3}<\cdots<R_{t}$ be the ordered sequence of $R(\chi)$; note that $R_{1}=R(\phi)$. Denote by $\delta_{k}, 1 \leqq k \leqq t$, the generator $\alpha\left(\mathscr{S}_{k}\right)$ defined by the elementary loop around the point $B\left(R_{k}\right)$ on the base point $B_{0}$, constructed according to the anticlockwise convention. For convenience we let $R_{t+1}$ be some real number satisfying $R_{t+1}>R_{t}$.

Definition 4.4.6. The subgroup $\mathscr{E}_{k}$ of $\mathscr{H}(r, s)$ is the subgroup generated by signatures $\alpha(\mathscr{S})$ such that, for some $B \in M(\mathscr{S}), x(B, \chi) \in \mathbb{P}^{s}$ satisfies $B\left(R_{k+1}\right)[x(B, \chi)]<0$. Here, as usual, $\chi=\varrho_{3} \cup \varrho_{4}$ for $\mathscr{S}=\left(\begin{array}{ll}\varrho_{1} & \varrho_{4} \\ \varrho_{3} & \varrho_{2}\end{array}\right)$; 
the condition means that some point on $M(\mathscr{S})$ corresponds to a pinch in the interior of the sphere $B\left(R_{k+1}\right)=0$. Note that $\phi=\mathscr{E}_{0} \subset \mathscr{E}_{1} \subset \cdots \subset \mathscr{E}_{t}$ $=\mathscr{H}(r, s)$.

Lemma 4.4.7. For all $k, 1 \leqq k \leqq t, \mathscr{E}_{k}$ is generated by $\delta_{1}, \ldots \delta_{k}$.

Proof. The proof is by induction on $k$. We suppose without loss of generality that $l_{j}(Y)>0$ for all $j$ (otherwise we replace $l_{j}$ by $-l_{j}$ ). All points $x$ such that $B\left(R_{2}\right)(x)<0$ also satisfy $l_{j}(x)>0$, for all $j$ (Fig. 4); hence $\mathscr{E}_{1}=\left\{\delta_{1}\right\}\left(=\left\{\alpha\left(\begin{array}{ll}\Omega & \phi \\ \phi & \phi\end{array}\right)\right\}\right)$.

Now (4.4.6) implies that $\mathscr{E}_{k+1}$ is generated by $\mathscr{E}_{k}$ together with all elements $\alpha(\mathscr{S})$ such that, for some $B \in M(\mathscr{S})$, the corresponding pinch point $x(B, \chi) \in \mathbb{P}^{s}$ satisfies

$$
B\left(R_{k+2}\right)(x(B, \chi))<0, \quad B\left(R_{k+1}\right)(x(B, \chi)) \geqq 0 .
$$

Let $\mathscr{S}$ be such a signature. The point $x(B, \chi)$ lies in a polygonal region of $l(\chi)$ bounded by planes $l_{j}, j \notin \chi$; if this region intersects the interior of $\left\{x \mid B\left(R_{k+1}\right)(\chi)=0\right\}$, then $\alpha(\mathscr{S}) \in \mathscr{E}_{k}$. The only other possibility is that the point $x\left(B\left(R_{k+1}\right), \chi_{k+1}\right)$ lies on the boundary of this region of $l(\chi)$. (Fig. 4 shows this situation in the case $s=2, r=3, k=6$. Signatures $\mathscr{S}$ for which the pinch point $x(B, \mathscr{S})$ lies in or on the boundary of the shaded region satisfy $\alpha(\mathscr{S}) \in \mathscr{E}_{7}-\mathscr{E}_{6}$ ). Thus $\mathscr{E}_{k+1}$ is obtained from $\mathscr{E}_{k}$ by adjoining generators $\alpha(\mathscr{S})$ for which some $B \in M(\mathscr{S})$ has a pinch point $x(B, \chi)$ arbitrarily close to $x\left(B\left(R_{k+1}\right), \chi_{k+1}\right)=x_{k+1}$.

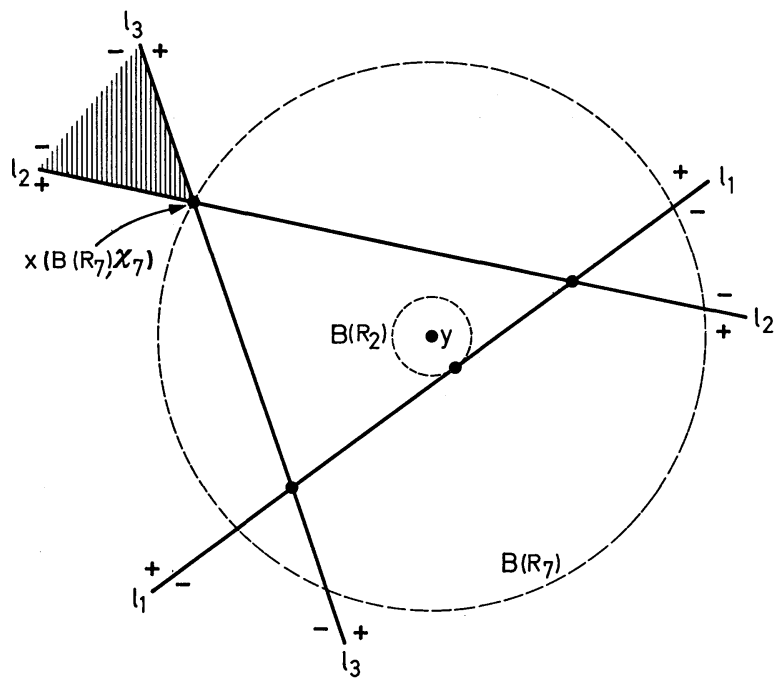

Fig. 4. The transition from $\mathscr{E}_{k}$ to $\mathscr{E}_{k+1}$ 
Now suppose $\mathscr{S}_{k+1}=\left(\begin{array}{ll}\eta_{1} & \eta_{4} \\ \eta_{3} & \eta_{2}\end{array}\right)\left(\right.$ recall $\left.\delta_{k+1}=\alpha\left(\mathscr{S}_{k+1}\right)\right)$. Let $\mathscr{T}$ be the reduced signature $\mathscr{T}=\left(\begin{array}{cc}\eta_{1} & \phi \\ \phi & \eta_{2}\end{array}\right)$, and define $\mathscr{K}=\{\alpha(\mathscr{S}) \mid \mathscr{T}$ is a reduced signature of $\mathscr{S}\}$ $\mathscr{K}^{\prime}=\left\{\alpha(\mathscr{S}) \in \mathscr{K} \mid \mathscr{S}=\left(\begin{array}{ll}\varrho_{1} & \varrho_{4} \\ \varrho_{3} & \varrho_{2}\end{array}\right), \quad\right.$ with $\left.\quad \eta_{3} \subset \varrho_{3} \cup \varrho_{1}, \eta_{4} \subset \varrho_{4} \cup \varrho_{2}\right\}$.

Our discussion above shows precisely that $\mathscr{E}_{k+1}$ is generated by $\mathscr{E}_{k}$ and $\mathscr{K}$. But $\mathscr{K}$ generates a subgroup of $\mathscr{H}(r, s)$ isomorphic to $\mathscr{K}\left(\left|\chi_{k+1}\right|\right)$; the elements of $\mathscr{K}^{\prime}$ are precisely the set of minimal generators of this subgroup discussed in 4.3. Thus $\mathscr{E}_{k+1}$ is generated by $\mathscr{E}_{k} \cup \mathscr{K}^{\prime}$.

Now $\delta_{k+1} \in \mathscr{K}^{\prime}$. Take $\alpha(\mathscr{S}) \in \mathscr{K}^{\prime}, \alpha(\mathscr{S}) \neq \delta_{k+1}$, with $\chi=\varrho_{3} \cup \varrho_{4}$, as usual; we will have proved the lemma once we have verified $\alpha(\mathscr{S}) \in \mathscr{E}_{k}$. To see this, choose points $z_{p} \in \mathbb{P}^{s}, p \in \chi_{k+1}$, satisfying $l_{j}\left(z_{p}\right)=\delta_{j p}$ for $j \in \chi_{k+1}$, and let $x=x_{k+1}+\varepsilon \sum_{\chi_{k+1}-\chi} \tau_{p}\left(B\left(R_{k+1}\right), \chi_{k+1}\right) z_{p}$. Then, for sufficiently small $\varepsilon$,

a) $\operatorname{sgn} l_{j}(x)=\operatorname{sgn} l_{j}\left(x_{k+1}\right), j \in \chi_{k+1}$;

b) $\operatorname{sgn} l_{j}(x)=\operatorname{sgn} \tau_{p}\left(B\left(R_{k+1}\right), \chi_{k+1}\right)$, if $j \in \chi_{k+1}-\chi$;

c) $B\left(R_{k+1}\right)(x)<0$, where c) is proved using (3.1.2). Then from a) and b) there is a $B \in M(\mathscr{S})$ with $x(B, \chi)=x$ (using 4.4.8); c) then implies $\alpha(\mathscr{S}) \in \mathscr{E}_{k}$.

We are now ready to prove the main theorem of this section.

Theorem 4.4.9. The conditions (i)-(iv) of 4.2 uniquely determine the trace of the representation $\mathscr{L}^{\prime}$ of $\mathbb{C}(\mathscr{H}(r, s))$.

Proof. For each of the subgroups $\mathscr{E}_{k}, 1 \leqq k \leqq t$, of $\mathscr{H}(r, s)$ defined by (4.4.6), denote by $\mathbb{C}_{1}\left(\mathscr{E}_{k}\right)$ the subring of $\overline{\mathbb{C}}(\mathscr{H}(r, s))$ generated by the elements $g-1, g \in \mathscr{E}_{k}$; we write $a_{k}=\delta_{k}-1$. We consider the trace of the subrepresentation $\mathscr{L}_{k}^{\prime}=\left.\mathscr{L}^{\prime}\right|_{\mathbb{C}_{1}\left(\mathscr{E}_{k}\right)}$. Since $\mathbb{C}(\mathscr{H}(r, s))=\mathbb{C}_{1}\left(\mathscr{E}_{t}\right) \oplus\{1\}$ and the trace of the unit element is known to be determined by (i)-(iv) (Lemma 4.4.3), it will suffice to prove that the trace of $\mathscr{L}_{k}^{\prime}$ is uniquely determined by (i)-(iv) for all $k, 1 \leqq k \leqq t$. The proof is by induction on $k$. For $k=1 \mathbb{C}_{1}\left(\mathscr{E}_{1}\right)$ is generated by $a_{1}$ and condition (i), together with its corollary Lemma 4.4.4, determine $\operatorname{tr} \mathscr{L}_{1}^{\prime}$ uniquely.

Now consider the inductive step. We suppose then that we have shown how to compute the trace of $\mathscr{L}^{\prime}(z)$ for any $z \in \mathbb{C}_{1}\left(\mathscr{E}_{k}\right)$. Now if $\alpha_{1} \ldots \alpha_{u}$ is any generating set for $\mathscr{E}_{k}$, the corresponding discontinuities $\alpha_{i}-1$ generate $\mathbb{C}_{1}\left(\mathscr{E}_{k}\right)$; we will make use of this freedom to replace the generators $\delta_{1} \ldots \delta_{k}$ of $\mathscr{E}_{k}$ with a more convenient set. This set contains generators of two types:

a) generators $\alpha(\mathscr{S})$, where $\mathscr{S} \in \mathscr{K}^{\prime}$ [see (4.4.8)] and $\varrho_{4} \cup \varrho_{3} \neq \chi_{k+1}$. 
b) generators $\alpha\left(\mathscr{S}^{\prime}\right)$ which correspond to the points of intersection with singularities $M\left(\chi_{k^{\prime}}\right), k^{\prime} \leqq k, \chi_{k^{\prime}} \downarrow \chi_{k+1}$, of the line $L$ in $W$ joining $B\left(R_{k+1}\right)$ and the point $B^{\prime}$ given by the quadratic form

$$
B^{\prime}(y)=\sum_{j=1}^{s}\left(y_{j}-x_{j}\left(B\left(R_{k+1}\right), \chi_{k+1}\right) y_{s+1}\right)^{2} .
$$

We remark that while the points $\delta_{1}, \ldots \delta_{t}$ were defined using a line representing a family of concentric spheres, the generators in b) above are defined using a family of spheres tangent to $B\left(R_{k+1}\right)$ at $x\left(B\left(R_{k+1}\right)\right.$, $\left.\chi_{k+1}\right)$ (Fig. 5). It may be shown as in Lemma 4.4.7 that a) and b) do define a set of generators for $\mathscr{E}_{k}$. Moreover, the generators in b) are all transverse to $\delta_{k+1}$; indeed, the intersection point of $L$ with $M\left(\chi_{k^{\prime}}\right), k^{\prime} \leqq k$, $\chi_{k^{\prime}} \downarrow \chi_{k+1}$, defining a generator $\alpha(\mathscr{S})$, is on the transverse intersection of $M\left(\mathscr{S}^{\prime}\right)$ and $M(\mathscr{S})$ (with $\delta_{k+1}=\alpha\left(\mathscr{S}^{\prime}\right)$ ).

Now from Lemma 4.4.7, $\mathscr{E}_{k+1}$ is generated by the generators of a) and b) together with $\delta_{k+1}$ - that is, by those of b) together with all generators $\alpha(\mathscr{S}) \in \mathscr{K}^{\prime}$. Consider a product of the discontinuity operators for this generating set. Since the trace of a product is equal to the trace of any other cyclic permutation of the same factors, and since condition (i) (4.2) gives $\mathscr{L}^{\prime}\left(a_{k+1}^{2}\right)$ as a multiple of $\mathscr{L}^{\prime}\left(a_{k+1}\right)$, there is no loss of generality in supposing that $\mathscr{L}^{\prime}\left(a_{k+1}\right)$ appears neither as first nor last factor of the product whose trace we wish to compute (except in the case in which $\mathscr{L}^{\prime}\left(a_{k+1}\right)$ is the only factor - in that case we use Lemma 4.4.4).

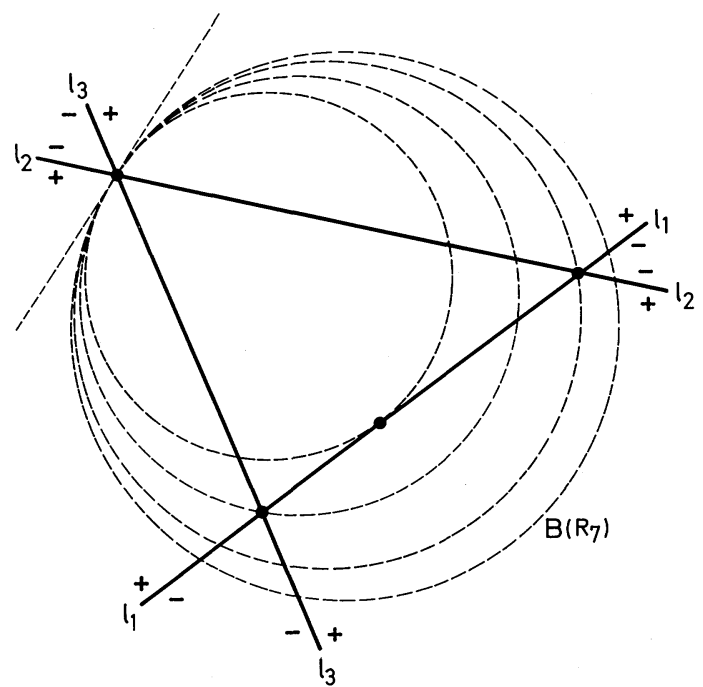

Fig. 5. Spheres used to define alternative generators for $\mathscr{E}_{k}$ 
Now each of the signatures $\mathscr{S}^{\prime}$ in the chosen minimal generating set which does not satisfy (4.4.8) is transverse to $\delta_{k+1}$. Therefore by (ii) (4.2) the product is either zero, or each time in which $\mathscr{L}^{\prime}\left(a_{k+1}\right)$ occurs it is in the form

$$
\mathscr{L}^{\prime}\left(a\left(\mathscr{S}^{\prime}\right)\right) \mathscr{L}^{\prime}\left(a_{k+1}\right) \mathscr{L}^{\prime}\left(a\left(\mathscr{S}^{\prime \prime}\right)\right),
$$

where $\mathscr{S}^{\prime}, \mathscr{S}^{\prime \prime}$ are in $\mathscr{K}^{\prime}$. But Remark 4.3.7 implies that (4.4.10) may be expressed as a multiple of the product

$$
\mathscr{L}^{\prime}\left(a\left(\mathscr{S}^{\prime}\right)\right) \mathscr{L}^{\prime}\left(a\left(\mathscr{S}_{0}\right)\right) \mathscr{L}^{\prime}\left(a\left(\mathscr{S}^{\prime \prime}\right)\right),
$$

where $\alpha\left(\mathscr{S}_{0}\right)=\alpha\left(\begin{array}{ll}\varrho_{1} & \phi \\ \phi & \varrho_{2}\end{array}\right)$ is in $\mathscr{K}^{\prime}$. But $\alpha\left(\mathscr{S}_{0}\right)$ is in $\mathscr{E}_{k}$ also; thus the trace may be calculated using the induction assumption.

\subsection{Construction of the Representation}

In this section we construct a representation $\mathscr{L}^{\prime \prime}$ of $\mathscr{H}(r, s)$, satisfying the conditions of 4.2, and irreducible; as observed in 4.1, this is then the unique representation satisfying these conditions, i.e. $\mathscr{L}^{\prime \prime}=\mathscr{L}^{\prime}$. The construction falls into two parts:

1. We construct the representation for $\mathscr{H}(r)=\mathscr{H}(r, s), r \leqq s$, satisfying the conditions of 4.2, and irreducible. To obtain candidates for the matrices of $\mathscr{L}^{\prime \prime}$ we assume the existence of this representation and exploit the conditions which we want $\mathscr{L}^{\prime \prime}$ to satisfy to come up with formulae for the matrix coefficients of $\mathscr{L}^{\prime \prime}(\alpha(\mathscr{S})), \mathscr{S}$ an arbitrary signature. It is then a simple exercise to check that the resulting matrices do define a representation of $\mathscr{H}(r)$ satisfying the conditions of 4.2 , and irreducible.

2. In the case $r>s$ we define a representation of $\mathscr{H}(r, s)$ by mapping it into $\mathscr{H}(r)$ (Remark 3.4.8 (A)) and taking the restriction to the image of $\mathscr{H}(r, s)$ in $\mathscr{H}(r)$ of the representation constructed in 1 . This representation of $\mathscr{H}(r, s)$ evidently satisfies (i)-(iii) of 4.2 since the representation $\mathscr{L}^{\prime \prime}$ of $\mathscr{H}(r)$ satisfies these conditions. To show that it satisfies (iv) we compute its dimension. Finally we show that it is irreducible.

Lemma 4.5.1. $\mathscr{L}^{\prime \prime}\left(\alpha\left(\begin{array}{ll}\phi & \phi \\ \phi & \phi\end{array}\right)\right)=-1$.

Proof. Since $\mathscr{L}^{\prime \prime}$ is required to be irreducible and $\alpha\left(\begin{array}{ll}\phi & \phi \\ \phi & \phi\end{array}\right)$ is in the center of $\mathscr{H}(r)(\operatorname{Remark} 3.4 .8(\mathrm{D}))$ it follows that $\mathscr{L}^{\prime \prime}\left(\alpha\left(\begin{array}{ll}\phi & \phi \\ \phi & \phi\end{array}\right)\right)$ is a multiple $c$ of the unit matrix acting in the representation space. To determine $c$ we show that

$$
\operatorname{tr} \mathscr{L}^{\prime \prime}\left(\alpha\left(\begin{array}{ll}
\phi & \phi \\
\phi & \phi
\end{array}\right)\right)=-2^{r-1}
$$


Since we have already shown (Lemma 4.4.3) that the conditions of 4.2 imply

$$
\operatorname{tr} \mathscr{L}^{\prime \prime}(1)=2^{r-1}
$$

it will follow that $c=-1$, as asserted. Now by definition ((3.3.21))

$$
\alpha\left(\begin{array}{ll}
\phi & \phi \\
\phi & \phi
\end{array}\right)=\prod_{\phi \subset \chi \subset \Omega} \alpha(\chi)
$$

where the $\alpha(\chi)=\alpha\left(\begin{array}{cc}\Omega-\chi & \chi \\ \phi & \phi\end{array}\right)$ are the minimal set of generators for $\mathscr{H}(r)$ used in 4.3, and the order of factors is such that $\alpha\left(\chi_{1}\right)$ stands to the left of $\alpha\left(\chi_{2}\right)$ if $\chi_{1} \supset \chi_{2}$. We apply $\mathscr{L}^{\prime \prime}$ to (4.5.3) and make use of the formulae of 4.3 to give

$$
\begin{aligned}
\mathscr{L}^{\prime \prime}\left(\alpha\left(\begin{array}{ll}
\phi & \phi \\
\phi & \phi
\end{array}\right)\right)= & +\sum_{\phi \subset \chi \subset \subset \Omega} \mathscr{L}^{\prime \prime}(a(\chi)) \\
& -\sum_{\substack{\phi \subset \chi_{1}, \chi_{2} \subset \Omega \\
\chi 1 \neq \chi_{2}}} \mathscr{L}^{\prime \prime}\left(a\left(\chi_{1}\right) a\left(\chi_{2}\right)\right)(-1)^{\left|\chi_{1}-\chi_{2}\right|} .
\end{aligned}
$$

We have $\operatorname{tr} \mathscr{L}^{\prime \prime}(a(\chi))=X(\chi)-1((4.4 .4))$ and (4.3.5) gives

$$
\operatorname{tr} \mathscr{L}^{\prime \prime}\left(a\left(\chi_{1}\right) a\left(\chi_{2}\right)\right)=-X\left(\chi_{2}\right) \prod_{i \in \chi_{1}-\chi_{2}}\left(1+Y_{i}\right) .
$$

We take traces in (4.5.4) and use these formulae to obtain (4.5.2), after simplification.

According to 4.2 (i) the representation space $E(r)$ for $\mathscr{L}^{\prime \prime}$ is to contain for each $\chi, \phi \subset \chi \subset \Omega$, a vector $u(\chi)$ which spans the range of $\mathscr{L}^{\prime \prime}(a(\chi))$. We normalize the $u(\chi)$ so that

$$
u(\chi)=(-1)^{r+|\chi|} X^{-1}(\chi) \mathscr{L}^{\prime \prime}(a(\chi)) u(\Omega), \quad \chi \neq \Omega .
$$

Since the $\mathscr{L}^{\prime \prime}(a(\chi))$ generate $\mathscr{L}^{\prime \prime}(\mathbb{C}(\mathscr{H}(r)))$ the vectors $u(\chi)$ span the representation space. It is convenient to work with the vectors $\Theta(\chi)$, $\phi \subset \chi \subset \Omega$, defined by

$$
\Theta(\chi)=\sum_{\psi \supset \chi} u(\psi)
$$

which also span the representation space. From the formulae of 4.3 we obtain

$$
\begin{aligned}
\mathscr{L}^{\prime \prime}(a(\eta)) \Theta(\chi)=\{ & \left.\sum_{\varrho \supset \eta}(-1)^{|\varrho-\eta|} \Theta(\varrho)\right\} \\
& \left\{-\delta_{\eta \supset \chi} \prod_{\eta-\chi}\left(-Y_{i}\right)+\delta_{\chi \supset \Omega-\eta} X(\eta)(-1)^{|\Omega-\eta|}\right\} .
\end{aligned}
$$


From (4.5.5) and the expression for $\alpha\left(\begin{array}{ll}\phi & \phi \\ \phi & \phi\end{array}\right)$ as a product of $\alpha(\chi)((4.5 .3))$ we obtain the formula

$$
\mathscr{L}^{\prime \prime}\left(\alpha\left(\begin{array}{ll}
\phi & \phi \\
\phi & \phi
\end{array}\right)\right) \Theta(\chi)=(-)^{|\chi|} X\left(\chi^{\prime}\right) \Theta\left(\chi^{\prime}\right),
$$

where $\chi^{\prime}=\Omega-\chi$. But by Lemma 4.5.1, $\mathscr{L}^{\prime \prime}\left(\alpha\left(\begin{array}{ll}\phi & \phi \\ \phi & \phi\end{array}\right)\right)=-1$, so

$$
\Theta(\chi)=-(-1)^{|\chi|} X\left(\chi^{\prime}\right) \Theta\left(\chi^{\prime}\right) .
$$

(4.5.7) shows that the vectors $\Theta(\chi)$ with $r \notin \chi$ span the representation space $E(r)$. However, there are only $2^{r-1}$ of these vectors and from (4.4.3) we know that $\mathscr{L}^{\prime \prime}$ should have dimension $2^{r-1}$. Hence the vectors $\Theta(\chi)$, $r \notin \chi$, must form a basis, and we therefore take the formulae which are obtained from (4.5.5) and (4.5.7) for $\mathscr{L}^{\prime \prime}(a(\eta))$ applied to vectors $\Theta(\chi)$, $r \notin \chi$, as a sum of these vectors as a definition of the matrix $\mathscr{L}^{\prime \prime}(a(\eta))$. The matrix $\mathscr{L}^{\prime \prime}(a(\mathscr{S})), \mathscr{S}$ as arbitrary signature, may then be computed from the expression for $\alpha(\mathscr{S})$ as a word in the minimal set of generators $\alpha(\chi)$. It is simplest to organize this calculation as anduction on the number of indices in $\varrho_{2} \cup \varrho_{4}$. (3.3.11) gives a formula for $\alpha(\mathscr{S})$ as a conjugate $\alpha\left(\mathscr{S}_{1}\right) \alpha\left(\mathscr{S}_{2}\right) \alpha\left(\mathscr{S}_{1}\right)^{-1}$ where $\mathscr{S}_{1}$ and $\mathscr{S}_{2}$ have one less index in $\varrho_{2} \cup \varrho_{4}$, e.g.

$$
a\left(\begin{array}{ll}
\varrho_{1} & \varrho_{4} \\
\phi & \varrho_{2}
\end{array}\right)=\alpha\left(\begin{array}{ll}
\varrho_{1} & \varrho_{4} \cup i \\
\phi & \varrho_{2}-i
\end{array}\right) a\left(\begin{array}{ll}
\varrho_{1} \cup i & \varrho_{4} \\
\phi & \varrho_{2}-i
\end{array}\right) \alpha\left(\begin{array}{ll}
\varrho_{1} & \varrho_{4} \cup i \\
\phi & \varrho_{2}-i
\end{array}\right)^{-1}
$$

for any $i \in \varrho_{2}$. Writing $\mathscr{L}^{\prime \prime}(a(\mathscr{S}))=u(\mathscr{S}) \otimes v(\mathscr{S})$, we obtain

$$
\begin{aligned}
u\left(\begin{array}{ll}
\varrho_{1} & \varrho_{4} \\
\varrho_{3} & \varrho_{2}
\end{array}\right)_{\psi}= & \delta_{\varrho_{4} \subset \psi \subset \Omega-\varrho_{3}}(-1)^{\left|\psi \cup \varrho_{2}\right|} \lambda\left(\psi \cap \varrho_{2}\right) \\
& -\delta_{\varrho_{3} \subset \psi \subset \Omega-\varrho_{4}} X(\phi)(-1)^{\left|\psi \cap \varrho_{2}\right|} \lambda\left(\psi \cup \varrho_{2}\right) \\
v\left(\begin{array}{ll}
\varrho_{1} & \varrho_{4} \\
\varrho_{3} & \varrho_{2}
\end{array}\right)_{\chi}= & (-1)^{\left|\varrho_{2}\right|+|x|+\left|\varrho_{4}\right|} \lambda\left(\varrho_{4}\right) \lambda^{-1}\left(\varrho_{2}\right) \\
& \cdot\left\{\delta_{\varrho_{1} \subset \chi \subset \Omega-\varrho_{2}} X(\Omega)(-1)^{\left|\chi \cap \varrho_{4}\right|} \lambda^{-1}\left(\chi \cup \varrho_{4}\right)\right. \\
& \left.-\delta_{\varrho_{2} \subset \chi \subset \Omega-\varrho_{1}}(-1)^{\left|\chi \cup \varrho_{4}\right|} \lambda^{-1}\left(\chi \cap \varrho_{4}\right)\right\}
\end{aligned}
$$

where $\lambda(\eta)=\prod_{i \in \eta} Y_{i}$ for any $\eta \subset \Omega$ (see (4.3.5)). The reader can now check that the formulae (4.5.8) and (4.5.9) furnish a representation $\mathscr{L}^{\prime \prime}$ of $\mathscr{H}(r)$ satisfying conditions (i)-(iv) of 4.2. The image $\mathscr{L}^{\prime \prime}(\mathbb{C}(\mathscr{H}(r)))$ is a complete 
matrix ring (this follows from the fact that $\left\{u\left(\begin{array}{cc}\Omega-\eta & \eta \\ \phi & \phi\end{array}\right)_{\psi} \mid r \in \eta\right\}$ and $\left\{v\left(\begin{array}{cc}\Omega-\eta & \eta \\ \phi & \phi\end{array}\right)_{\chi} \mid r \notin \eta\right\}$ are easily seen to be linearly independent as vectors in $\left.\mathbb{C}^{2 r-1}\right)$, hence $\mathscr{L}^{\prime \prime}$ is irreducible.

For an arbitrary reduced signature

$$
\mathscr{T}=\left(\begin{array}{ll}
\varrho_{1} & \varrho_{4} \\
\varrho_{3} & \varrho_{2}
\end{array}\right) \quad \text { with } \quad \varrho_{1} \cup \varrho_{2} \cup \varrho_{3} \cup \varrho_{4}=\tau \subset \Omega
$$

we obtain the formula $(a(\mathscr{T})=\alpha(\mathscr{T})-1)$

$$
\begin{aligned}
\mathscr{L}^{\prime \prime}\left(a\left(\begin{array}{ll}
\varrho_{1} & \varrho_{4} \\
\varrho_{3} & \varrho_{2}
\end{array}\right)\right)_{\psi, \chi}= & (-)^{|\chi-\psi|} \delta_{\psi \Delta \chi^{\prime} \subset \tau}\left\{\delta_{\varrho_{4} \subset \psi \subset \varrho_{3}^{\prime}} \delta_{\varrho_{1} \subset \chi \subset \varrho_{2}^{\prime}} \mathrm{X}(\Omega)\right. \\
& \cdot \lambda^{-1}\left(\varrho_{2}-\psi\right) \lambda^{-1}\left(\chi-\varrho_{4}\right) \\
& \left.+\delta_{\varrho_{3} \subset \psi \subset \varrho_{4}^{\prime}} \delta_{\varrho_{2} \subset \chi \subset \varrho_{1}^{\prime}} X(\phi) \lambda\left(\psi-\varrho_{2}\right) \lambda\left(\varrho_{4}-\chi\right)\right\} \\
& -(-)^{|\psi-\chi|} \delta_{\psi \Delta \chi \subset \tau}\left\{\delta_{\varrho_{4} \subset \psi \subset \varrho_{3}^{\prime}} \delta_{\varrho_{2} \subset \chi \subset \varrho_{1}^{\prime}} \lambda^{-1}\left(\varrho_{2}-\psi\right)\right. \\
& \left.\cdot \lambda\left(\varrho_{4}-\chi\right)+\delta_{\varrho_{3} \subset \psi \subset \varrho_{4}^{\prime}} \delta_{\varrho_{1} \subset \chi \subset \varrho_{2}} \lambda\left(\psi-\varrho_{2}\right) \lambda^{-1}\left(\chi-\varrho_{4}\right)\right\}
\end{aligned}
$$

which is equivalent to (4.5.9) in the case $\tau=\Omega$ (here again $\chi^{\prime}=\Omega-\chi$ ).

We now obtain the (irreducible) representation $\mathscr{L}^{\prime \prime}(r, s)$ of $\mathscr{H}(r, s)$ as a sub-representation of $\mathscr{L}^{\prime \prime}$. More precisely, we define $\mathbb{C}_{1}(\mathscr{H}(r, s))$ to be the sub-ring of $\mathbb{C}(\mathscr{H}(r, s))$ spanned by discontinuities $a(\mathscr{S})$, set $E(r, s)=\mathscr{L}^{\prime \prime}\left(\mathbb{C}_{1}(\mathscr{H}(r, s))\right) E(r)$, and define $\mathscr{L}^{\prime \prime}(r, s)$ to be $\mathscr{L}^{\prime \prime}(\mathscr{H}(r, s))$ restricted to the vector space $E(r, s)$. (We again note that $E(r, s)$ depends implicitly on the positional characteristic introduced in Definition 3.3.7.) $\mathscr{L}^{\prime \prime}(r, s)$ clearly satisfies the conditions (i)-(iii) of 4.2 ; it remains only to calculate its dimension and show that it is irreducible.

Lemma 4.5.11. For any $\chi \subset \Omega$, define $w(\chi)=u\left(\begin{array}{cc}\Omega-\chi & \phi \\ \phi & \chi\end{array}\right) \in E(r)$. Then $E(r, s)$ is spanned by

$$
\left\{w(\chi) \mid\left(\begin{array}{cc}
\Omega-\chi & \phi \\
\phi & \chi
\end{array}\right) \text { is admissible in } \mathscr{H}(r, s)\right\} .
$$

Proof. We have to prove that for any admissible $\mathscr{S}=\left(\begin{array}{ll}\varrho_{1} & \varrho_{4} \\ \varrho_{3} & \varrho_{2}\end{array}\right)$ the vector $u(\mathscr{S})$ is in the span of the vectors (4.5.12). The proof is by induction on $k=\left|\varrho_{3}\right|+\left|\varrho_{4}\right|$. For $k=0, u(\mathscr{S})$ is one of the vectors (4.5.12) so the proposition holds trivially. For $k>0$ we have, say, $\varrho_{3} \neq \phi$. Let $j$ be any 
index in $\varrho_{3}$, and write $\mathscr{S}={ }_{j} \mathscr{T}$. Then

$$
\alpha^{-1}\left(\mathscr{T}_{j}\right) a(\mathscr{S}) \alpha\left(\mathscr{T}_{j}\right)=\alpha(j \mathscr{T}) a(\mathscr{S}) \alpha^{-1}\left({ }^{j} \mathscr{T}\right)
$$

- for both sides of the equation are equal to $a\left(\mathscr{T}^{j}\right)$. (4.5.13) implies that the vectors

$$
\alpha^{-1}\left(\mathscr{T}_{j}\right) u(\mathscr{S}) \text { and } \alpha(\dot{T}) u(\mathscr{S})
$$

are proportional. The first of these vectors is a linear combination of $u(\mathscr{S})$ and $u\left(\mathscr{T}_{j}\right)$, and the second of $u(\mathscr{S})$ and $u\left(\mathscr{T}^{j}\right)$. Since $u\left(\mathscr{T}_{j}\right)$ and $u\left({ }^{j} \mathscr{T}\right)$ are not proportional (cf. (4.5.8)) we obtain an expression for $u(\mathscr{S})$ as a linear combination of $u\left(\mathscr{T}_{j}\right), u\left({ }^{j} \mathscr{T}\right)$. But $\mathscr{T}_{j}$ and ${ }^{j} \mathscr{T}$ are admissible signatures with $\left|\varrho_{3}\right|+\left|\varrho_{4}\right|=k-1$ so by the induction assumption $u\left(\mathscr{T}_{j}\right), u\left({ }^{j} \mathscr{T}\right)$ lie in the span of the vectors (4.5.12). Hence $u(\mathscr{S})$ lies in this span.

Lemma 4.5.14. The vectors $w(\chi), \chi \subset \Omega, r \notin \chi$, are linearly independent.

Proof. $w(\chi)_{\psi}=(-1)^{|\psi \cup \chi|} \lambda(\psi \cap \chi)-X(\phi)(-1)^{|\psi \cap \chi|} \lambda(\psi \cup \chi)((4.5 .8))$.

Since $r \notin \psi$ and $r \notin \chi$ the $\lambda$ factors in this equation involve only the $Y_{i}$, $i \neq r$. Our assumption that the $\lambda_{i}$ are generic implies that $X(\phi)$ and these $Y_{i}$ are algebraically independent. The determinant of the matrix $\left\{w(\chi)_{\psi}\right\}$ can therefore vanish (for generic $\lambda_{i}$ ) only if it does so for $X(\phi)=0$. However, in that case the determinant does not vanish since its expansion contains exactly one term of maximum total degree in the $Y_{i}$, obtained by taking the product of the diagonal coefficients.

Lemma 4.5.15. There are precisely $d(r, s)$ vectors (4.5.12).

Proof. There is a natural $1: 1$ correspondence between the vectors (4.5.12) and the connected regions into which $\mathbb{P}^{s}$ is divided by the $l_{j}$. The number of these regions $N(r, s)$ depends only on $r$ and $s$, and may be calculated by deriving the recursion formula

and noting that

$$
N(r, s)=N(r-1, s)+N(r-1, s-1)
$$

$$
N(r, s)=\left\{\begin{array}{lll}
1 & \text { if } & r=1 \\
r & \text { if } & s=1
\end{array}\right.
$$

(see [13]). It follows that $N(r, s)=d(r, s)$, defined by (4.4.2).

Lemma 4.5.16. $\mathscr{L}^{\prime \prime}(r, s)(\mathbb{C}(\mathscr{H}(r, s)))$ is a complete matrix ring of dimension $d(r, s)$; and hence $\mathscr{L}^{\prime \prime}(r, s)$ is irreducible.

Proof. For admissible $\mathscr{S}=\left(\begin{array}{cc}\Omega-\chi & \phi \\ \phi & \chi\end{array}\right)$, we write (in $\left.E(r)\right)$

$$
a(\mathscr{S})=w(\chi) \otimes z(\chi),
$$


with $z(\chi) \in E(r)^{*}$. By Lemmas 4.5.11 and 4.5.14 the vectors $w(\chi)$ (i.e., the vectors of 4.5.12) form a basis in $E(r, s)$. Define $z^{\prime}(\chi) \in E(r, s)^{*}$ by

$$
\left(z^{\prime}(\chi), w\right)=(z(\chi), w), \quad(\text { any } \quad w \in E(r, s)) .
$$

From (4.5.8) and (4.5.9) one calculates that for admissible $\mathscr{S}=\left(\begin{array}{cc}\Omega-\chi & \phi \\ \phi & \chi\end{array}\right)$, $\mathscr{S}^{\prime}=\left(\begin{array}{cc}\Omega-\psi & \phi \\ \phi & \psi\end{array}\right)$ one has $\left(z^{\prime}(\chi), w(\psi)\right) \neq 0$. It then suffices to show that $\left\{z^{\prime}(\chi) \mid r \notin \chi\right\}$ are linearly independent, since this implies the linear independence of the $d(r, s)^{2}$ operators $\alpha(\mathscr{S}) \alpha\left(\mathscr{S}^{\prime}\right)$. Thus we must show that the matrix $\left\{\left(z^{\prime}(\chi), w(\psi)\right) \mid r \notin \chi, \psi\right\}$ is nonsingular. Since

$$
\left(z^{\prime}(\chi), w(\psi)\right)=\left\{(-)^{|\psi \cup \chi|} \lambda(\psi \cap \chi)-X(\phi)(-)^{|\psi \cap \chi|} \lambda(\psi \cup \chi)\right\} \lambda(\chi)(-)^{|\psi|},
$$

this matrix is equivalent to a submatrix of the one considered in Lemma 4.5.14; the proof of nonsingularity is similar.

We have now completed our construction of an irreducible representation $\mathscr{L}^{\prime \prime}(r, s)$ of $\mathscr{H}(r, s)$ which satisfies conditions (i)-(iv) of 4.2. Since only these conditions were used in the calculation of traces (4.4), we know that $\mathscr{L}^{\prime \prime}(r, s)$ has the same trace as the representation $\mathscr{L}^{\prime}(r, s)$ generated by the integral (2.5) Thus Lemma 4.1.1 implies that $\mathscr{L}^{\prime \prime}$ and $\mathscr{L}^{\prime}$ are equivalent.

Remark 4.5.17. Recall we write $H_{\gamma}$ for the function (2.5) defined by the integration cycle $\gamma$. We have actually proved the above result only for $\gamma$ of the type discussed in Remark 1 of 4.1 (see also Appendix $A$, where a cycle $\gamma_{0}$ of this type was constructed). But now suppose that $\gamma$ is arbitrary except for the condition that $H_{\gamma}$ be non-trivial. Then there is some signature $\mathscr{S}$ such that $a(\mathscr{S}) \mathscr{H}_{\gamma} \neq 0$, i.e., that $H_{e(\mathscr{S})}$ is in the vector space $V(\gamma)$ generated by $H_{\gamma}$ (here $e(\mathscr{S})$ is the vanishing cycle for $\left.\mathscr{S}\right)$. But $H_{e(\mathscr{S})}$ is also in $V\left(\gamma_{0}\right)$; moreover, the fact that $\mathscr{L}^{\prime}(r, s)(\mathbb{C}(\mathscr{H}(r, s)))$ is a complete matrix ring implies that $H_{e(\mathscr{G})}$, and hence $H_{\gamma}$, is singular on every $M(\chi)$. That is, $\gamma$ is actually of the required type, and generates the same representation $\mathscr{L}^{\prime}(r, s)$. We have also shown that any two nontrivial cycles $\gamma$ and $\gamma^{\prime}$ actually generate the same (multi-valued) function.

Remark 4.5.18. For real generic values of the parameters $\lambda_{i}$ there is a hermitian scalar product on the representation space $E(r)$, uniquely defined up to a factor, with respect to which the representation $\mathscr{L}^{\prime \prime}(r)$ of $\mathscr{H}(r)$ is "unitary". The representation $\dot{\mathscr{L}}^{\prime \prime}(r, s)$ of $\mathscr{H}(r, s)$ is then "unitary" with respect to the induced scalar product on $E(r, s)$. To describe the scalar product on $E(r)$ we regard it as a non-singular antilinear map $\tau: E(r) \rightarrow E(r)^{*}$. Since the vectors $u(\chi)=u\left(\begin{array}{cc}\Omega-\chi & \chi \\ \phi & \phi\end{array}\right)$ span $E(r)$ it suffices 
to define $\tau$ on these vectors. We have

$$
\tau(u(\chi))=g_{\chi} v(\chi)
$$

with

$$
g_{\chi}=X^{-\frac{1}{2}}(\chi) e^{i \pi / 2} c_{\chi}
$$

and

$$
\begin{aligned}
& c_{\phi}=1, \\
& c_{\chi}=2^{|\chi|} \prod_{i \in \chi}\left(\sin \pi \lambda_{i}\right)^{-1}, \quad \chi \neq \phi .
\end{aligned}
$$

\subsection{The Problem of Contracting a Line}

In [1] we proved an isomorphism theorem which described the effect on the monodromy ring of cutting a line of one of the self-energy graphs studied in that paper, and remarked that this theorem might be generalized to arbitrary graphs. For the single loop graphs studied in the present paper this operation is trivial but the operation of contracting a line (which is trivial for the self-energy graphs) is not. In this section we note the implication of our results on this question. The discussion will be confined to the case 3 of integrals not having second-kind singularities. As will be shown in $\S 5.1$ the fundamental group in this case is $\mathscr{G}(N, N-1)$, the corresponding group defined algebraically $\mathscr{H}(N, N-1)$. (Definition 3.4.1.) We will distinguish quantities related to $\mathscr{H}(N-1, N-2)$ from quantities related to $\mathscr{H}(N, N-1)$ by primes. Thus the generators of $\mathscr{H}(N-1, N-2)$ will be written $\alpha^{\prime}\left(\begin{array}{ll}\varrho_{1} & \varrho_{4} \\ \varrho_{3} & \varrho_{2}\end{array}\right)$ where $\varrho_{1} \cup \varrho_{2} \cup \varrho_{3} \cup \varrho_{4}=\Omega_{N}$.

There is an evident homomorphism $h: \mathscr{H}(N-1, N-2) \rightarrow \mathscr{H}(N, N-1)$ which maps a generator $\alpha^{\prime}(\mathscr{S})$ of $\mathscr{H}(N-1, N-2)$ into the element $\alpha(\mathscr{S})$ of $\mathscr{H}(N, N-1)$. The image of $\mathscr{H}(N-1, N-2)$ under $h$ is the subgroup of $\mathscr{H}(N, N-1)$ generated by the elements $\alpha(\mathscr{S}), \mathscr{S}$ a reduced signature not containing the index $N$.

Theorem 4.6.1. The representation $\mathscr{L}_{N}^{\prime}(\lambda) h$ of $\mathscr{H}(N-1, N-2)$ is the direct sum of the representations $\mathscr{L}_{N-1}^{\prime}\left(\lambda^{(+)}\right), \mathscr{L}_{N-1}^{\prime}\left(\lambda^{(-)}\right)$, i.e. of two copies of the generic representation of $\mathscr{H}(N-1, N-2)$ for different parameter values. The parameter values $\lambda^{+}, \lambda^{-}$are given via the corresponding $X^{+}, X^{-}$by

$$
X^{ \pm}(\chi)= \pm X(\chi) \sqrt{-Y_{N}} \text { for } \chi \subsetneq \Omega_{N} .
$$


Proof. The element $\beta_{N}=\alpha\left(\begin{array}{ll}N & \phi \\ \phi & \phi\end{array}\right)$ commutes with $h \mathscr{H}(N-1, N-2)$. Hence $\mathscr{L}_{N}^{\prime}(\lambda) h$ is the direct sum of representations of $\mathscr{H}(N-1, N-2)$ acting in the eigenspaces of $\mathscr{L}_{N}^{\prime}\left(\beta_{N}\right)$. Now $\left[\mathscr{L}_{N}^{\prime}\left(\beta_{N}\right)\right]^{2}$ is a multiple of the identity (cf. Remark 3.4.8 (D)) so there are just two of these eigenspaces. The corresponding eigenvalues (computed from 4.5.10) are $\pm \sqrt{-Y_{N}^{-1}}$. Using (4.5.10) and the expression for the word at infinity obtained in Appendix B it may be verified that each of the representations into which $\mathscr{L}_{N}^{\prime}(\lambda) h$ splits satisfies the conditions which characterize the representation $\mathscr{L}_{N-1}^{\prime}$ of $\mathscr{H}(N-1, N-2)(4.2)$, the corresponding values of the parameters being given by (4.6.2).

In order to understand the meaning of the homomorphism $h$ on the geometrical level we seek a homomorphism $k$ such that the diagram

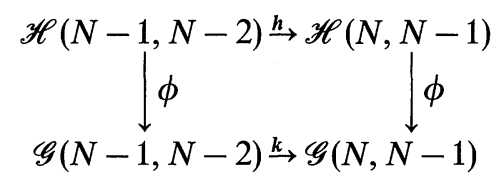

commutes. To construct $k$ we define an embedding $k^{\prime}$ of the parameter space $\left\{B_{i j}^{\prime}: 1 \leqq i, j<N\right\}$ for $\mathscr{G}(N-1, N-2)$ into the parameter space $\left\{B_{i j}: 1 \leqq i, j \leqq N\right\}$ for $\mathscr{G}(N, N-1)$ by

$$
\left.\begin{array}{rlrl}
k^{\prime}\left(B^{\prime}\right)_{i j} & =B_{i j}^{\prime} & & 1 \leqq i, j<N \\
& =1 & & i=j=N \\
& =0 & & \text { otherwise }
\end{array}\right\} .
$$

$k$ is then the homomorphism of fundamental groups induced by $k^{\prime}$. It is easily seen to have the desired properties. Unfortunately the construction of $k^{\prime}$ does not appear to have an immediate analog for more general graphs so we do not have a conjecture on the effect of contracting a line in a general graph.

\section{§ 5. Applications of the Representation Theory}

\subsection{Relation of Generic and Physical Integrals}

In $\S 2$ we distinguished four cases in our study of the integral (2.2). In this section we discuss the first three of these cases, applying our representation theory of the generic integral (2.5) to obtain the monodromy rings of the integrals in question. For the moment we restrict our attention to cases 2) and 3), in which no renormalization is necessary.

For these cases we write the integral (2.2) in the form (2.4), and eliminate the $\delta$-function by performing the $\alpha_{N+1}$ integral. Then (2.4) 
becomes

$$
F(A)=\Gamma\left(N-\frac{m}{2}\right) \int_{\alpha_{i} \geqq 0} \frac{\left(-\sum_{i=1}^{N} \alpha_{i}\right)^{N-m}}{D(\alpha, A)^{N-m / 2}} \eta^{N-1}(\alpha) .
$$

For the moment we ignore the $\Gamma$-function in (5.1.1). It is convenient to think of obtaining (5.1.1) from (2.5) in two steps. In the first we keep the parameters $\lambda_{j}$ arbitrary, but take

and in case 3 ,

$$
\begin{array}{rlrl}
s & =N-1, & \\
B_{i j} & =A_{i j} & & (i, j=1, \ldots N), \\
l_{j}(\alpha) & =\alpha_{j} & & (j=1, \ldots N),
\end{array}
$$

while in case 2 ,

$$
\begin{aligned}
r & =N, \\
P(\alpha) & =\left(-\sum_{i=1}^{N} \alpha_{i}\right)^{N-m},
\end{aligned}
$$

$$
\begin{aligned}
r & =N+1, \\
l_{N+1}(\alpha) & =-\sum_{i=1}^{N} \alpha_{i}, \\
P(\alpha) & =1 .
\end{aligned}
$$

We will assume that (5.1.2) and either (5.1.3) or (5.1.4) holds from now on. The second step is to specialize the parameters $\lambda_{j}$ :

$$
\lambda_{j}=0 \quad(j=1, \ldots N)
$$

and, in case $2, \lambda_{N+1}=N-m$.

There remains to consider only the integration cycle to be used in (2.5). Let $\delta_{1}$ be the (relative) cycle of (5.1.1):

$$
\delta_{1}=\left\{\alpha \in \mathbb{P}^{N-1} \mid \alpha_{i} \geqq 0, i=1, \ldots N\right\} .
$$

Then $H_{\delta_{1}}($ see $(2.5))$ is well defined whenever $\lambda_{j}>-1, j=1, \ldots N$. Moreover, when $\lambda_{j}$ is non-integral, $j=1, \ldots N$, we may write

$$
H_{\delta_{1}}=\left\{\prod_{1}^{N}\left(e^{2 \pi i \lambda_{j}}-1\right)^{-1}\right\} H_{\delta_{0}},
$$

where $\delta_{0}$ is a closed cycle on which the integrand of (2.5) is single valued, and such that the support of $\delta_{0}$ is arbitrarily close to $\delta_{1}$ [10]. The representation theory derived in $\S 3$ and $\S 4$ thus applies to $H_{\delta_{1}}$; we must study the behaviour of this representation when the $\lambda$ 's are specialized. 
Note that since the singularity surfaces are independent of the $\lambda$ 's, the discussion of $\S 3$ applies to the physical integrals. In particular, we may label the singularity curves of the physical integrals by the same signatures.

Remark 5.1.5. The reader should verify that the conditions (5.1.2), together with either (5.1.3) or (5.1.4), give rise to the positional characteristics discussed in Remarks 3.4.3(A) and 3.4.4(B) respectively. Thus, in Case 3, all signatures $\mathscr{S}=\left(\begin{array}{ll}\varrho_{1} & \varrho_{4} \\ \varrho_{3} & \varrho_{2}\end{array}\right)$ in which $\varrho_{1} \cup \varrho_{2} \neq \phi$ occur; in Case 2, all signatures occur in which $\varrho_{1} \neq \phi, \varrho_{2} \neq \phi$. In Case 2, a curve is a second type singularity when it comes from a pinch involving $l_{N+1}$, that is, $\mathscr{S}$ is the signature for a second type singularity iff $N+1 \in \varrho_{3} \cup \varrho_{4}$.

Definition 5.1.6. Let $\Psi$ be the germ at $A_{0}\left(=B_{0}\right)$ of the integral (2.5) [Case 2 or 3] taken over $\delta_{0}$. Then $\Psi$ is called the physical sheet.

We have defined the representations (4.5) in an abstract way, with no reference to any particular contour. But we have

Lemma 5.1.7. The physical sheet $\Psi$ satisfies

$$
\mathscr{L}(a(\mathscr{S})) \Psi=0
$$

unless $\mathscr{S}=\left(\begin{array}{ll}\varrho_{1} & \varrho_{4} \\ \varrho_{3} & \varrho_{2}\end{array}\right)$ satisfies

a) in Case 3, $\varrho_{1}=\phi$ or $\varrho_{2}=\phi$,

b) in Case $2, \varrho_{1}=\{N+1\}$ or $\varrho_{2}=\{N+1\}$.

These conditions suffice to determine $\Psi$ uniquely as a vector in the representation space $E(r, s)$ (see 4.5$)$.

Proof. Conditions a) and b) are called positive $\alpha$ conditions; they express the fact that the pinch must take place on the contour $\delta_{1}$. One verifies the first part of the Lemma by the techniques of Appendix A; specifically, one may find, for any $\mathscr{S}$ not satisfying a) (resp. b)), a (nongeneric) real line $A(t)$ in the parameter space such that $A(0)=A_{0}$, $A\left(t_{1}\right) \in M(\mathscr{S})$ for some $t_{1}>0$, and $\left\{\alpha \mid A(t)(\alpha)=0,0 \leqq t \leqq t_{1}\right\} \cap \delta_{1}=\phi$. The second part of the lemma follows directly from (4.5.9).

A natural question arising from the above is the connection between our physical sheet as a germ at $A_{0}$ and the usual physical sheet. Let $A_{1}$ be any real quadratic form not lying on any singular curve, and define $A_{1, \varepsilon}$ as the form

$$
A_{1, \varepsilon}(\alpha)=\sum_{i, j=1}^{s+1}\left(A_{1}\right)_{i j} \alpha_{i} \alpha_{j}-i \varepsilon\left[\sum_{1}^{s+1} \alpha_{j}\right]^{2}
$$


Then the usual physical sheet at the point $A_{1}$ is

$$
\Psi\left(A_{1}\right)=\lim _{\varepsilon \rightarrow 0+} F\left(A_{1, \varepsilon}\right)
$$

(see (5.1.1)). Of course, this agrees with $\Psi$ at $A_{0}$.

Now consider the line $A_{0} A_{1}$ parameterized by

$$
A(t)=(1-t) A_{0}+t A_{1} .
$$

Let $\beta:[0,1] \rightarrow \mathbb{C}$ be a path such that $\gamma=A(\beta(s))$ is a path from $A_{0}$ to $A_{1}$ which is drawn by the counterclockwise convention. Then

Lemma 5.1.8. The physical sheet $\Psi\left(A_{1}\right)$ is the complex conjugate of the analytic continuation of $\Psi$ along $\gamma$ :

$$
\Psi\left(A_{1}\right)=\overline{[\gamma \Psi]} .
$$

Proof. Take $\varepsilon<0$, and consider the line

$$
A_{\varepsilon}(t)=(1-t) A_{0, \varepsilon}+t A_{1, \varepsilon} .
$$

Let $\gamma_{1}(s)=A_{0, s \varepsilon}$ and $\gamma_{2}(s)=A_{1,(1-s) \varepsilon}(s \in[0,1])$; for sufficiently small $\varepsilon$ the composite path

$$
\gamma^{\prime}=\gamma_{2} A_{\varepsilon}(\beta) \gamma_{1}
$$

is homotopic to $\gamma$. But for $\operatorname{Re} t \geqq 0, \operatorname{Im} t \leqq 0$ the quadric $\left\{A_{\varepsilon}(t)=0\right\}$ does not intersect $\delta_{1}=\left\{\alpha \mid \alpha_{i} \geqq 0\right\}$, so we may continue $\Psi$ along $A_{\varepsilon}(\beta) \gamma_{1}$ without

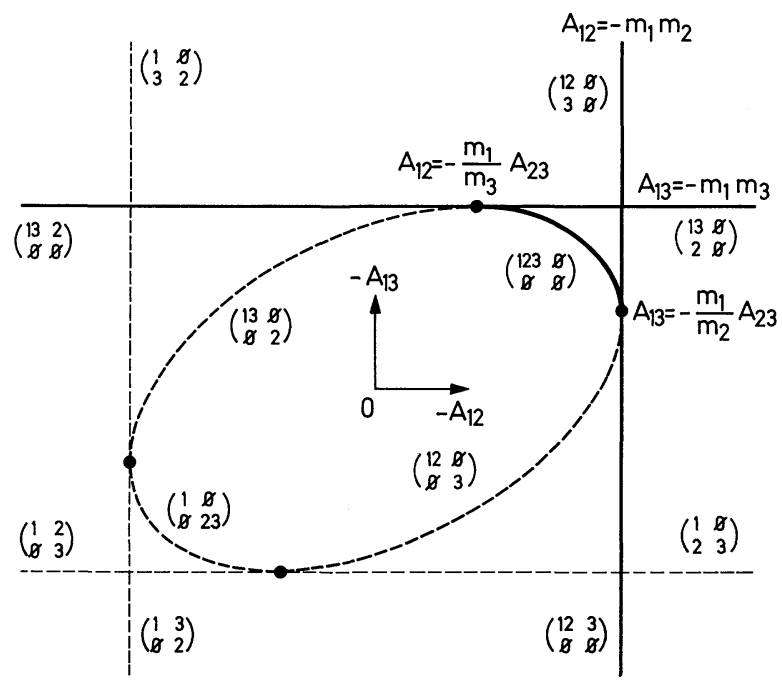

Fig. 6. Landau curves of the triangle graph (without second kind singularities) 


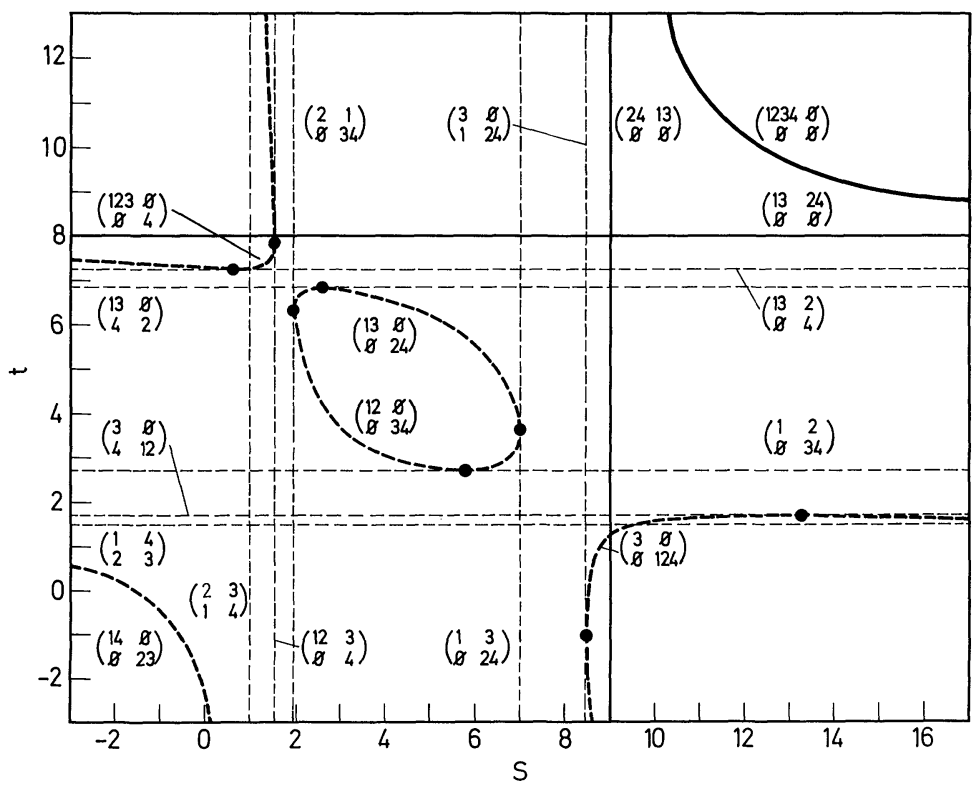

Fig. 7. Landau curves of the square graph

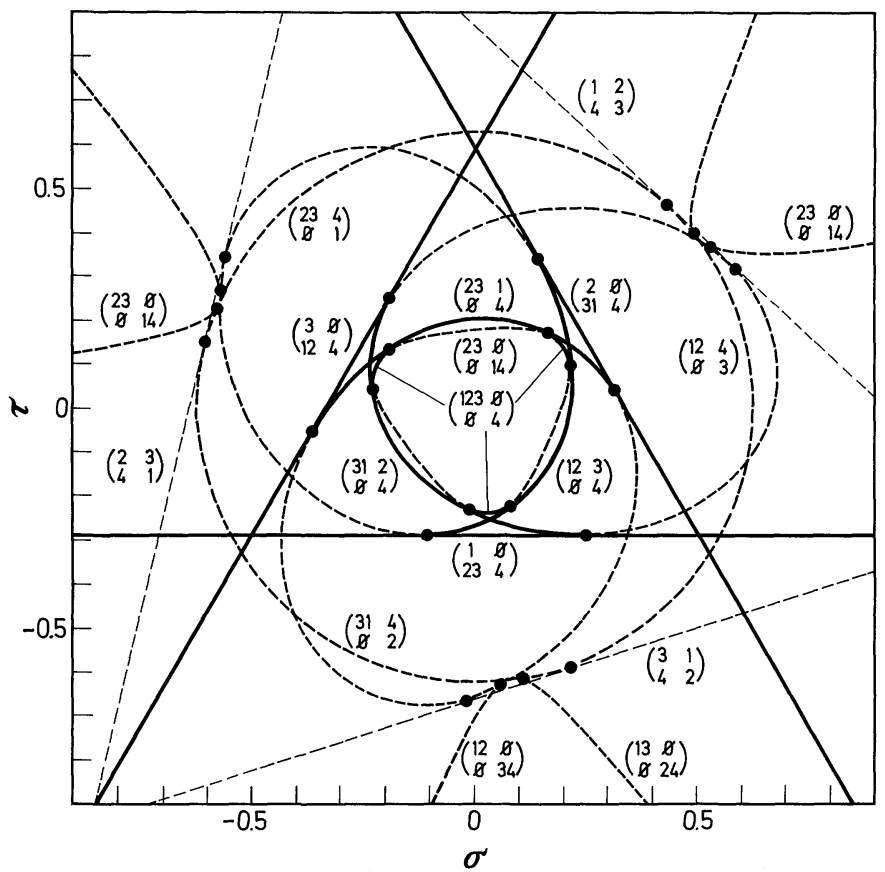

Fig. 8. Landau curves of the triangle graph (with second kind singularities) 
distorting the initial contour. Thus

$$
\overline{\Psi\left(A_{1}\right)}=\lim _{\varepsilon \rightarrow 0-} F\left(A_{1, \varepsilon}\right)=\gamma^{\prime} \Psi=\gamma \Psi .
$$

In order to exemplify the results of previous sections, we show in Figs. 6-8 the real two-dimensional section of Landau curves and their corresponding signatures for the simplest nontrivial cases.

In Fig. 6, which corresponds to $N=m=3, m_{1} m_{2}, m_{3}$ are arbitrary positive constants while $0<A_{23}<m_{2} m_{3}$ and $A_{12}, A_{13} \in \mathbb{R}$. Clearly, the variety $M(1)$ is confined at infinity in the plane spanned by $A_{12}, A_{13}$. The signatures for $M(2), M(3), M(\phi)$ are easily computed from (3.1.2), (3.2.1); according to Lemma 5.1.7, the heavy lines are actual singularities for the physical sheet. This case corresponds to Fig. 2.3.3a of [5]. In Fig. 6, as well as in Figs. 7, 8, the base point of the fundamental group is placed at the origin of the coordinates.

Fig. 7 corresponds to $N=m=4$, with values of the masses associated to internal and external lines of the Feynman graph which satisfy the following relations: $m_{i j}<m_{i}+m_{j}$ for $i j=12,23,34,41$, where $m_{i, i+1}^{2}=k_{i, i+1}^{2}{ }^{1}$. Since all masses are fixed, the varieties $M(123), M(124), M(134), M(234)$, $M(12), M(14), M(23), M(34)$ do not show up in the affine plane spanned by the Mandelstam variables $s, t$. In order to avoid confusion, the signatures of only part of the branches shown in Fig. 7 have been displayed; the reader can retrieve the missing signatures by means of Lemma 3.3.10. Fig. 7 corresponds to Fig. 2.4.4 of [5].

In Fig. 8, the case $N=m-1=3$ is considered when all masses depend linearly on $\sigma, \tau \in \mathbb{R}^{2}$ All Landau curves, of first and second type, are shown. Note that this section is, in the parameter space, not far from conical points of $M(\phi)$ as shown by the signatures of the cubic. If the varieties $M(14), M(24), M(34), M(4)$ are disregarded and the index 4 is dropped from the remaining signatures, we obtain a generic section for the case $N=m=3$, which contains in particular Fig. 6 .

\subsection{Specialization of Parameters - Case 3}

In this section we study the integral without second kind singularities $\left[(2.5)\right.$ with (5.1.2) and (5.1.3)] under the specialization $\lambda_{j}=0, j=1, \ldots N$. The key remark is

Lemma 5.2.1. Let $\Psi(\lambda)$ be the physical sheet (Definition 5.1.6), and let $h(\lambda) \in \mathbb{C}(\mathscr{G}(N, N-1))$ be a finite sum

$$
h(\lambda)=\sum_{\alpha \in \mathscr{G}(N, N-1)} k_{\alpha}(\lambda) \alpha,
$$

1 The actual parameters of Fig. 7 are: $A_{11}=0.643, A_{22}=1.00, A_{33}=4.12, A_{44}=4.00$, $A_{12}=0.131, A_{14}=1.13, A_{23}=1.16, \quad A_{34}=-0.415, \quad A_{13}=2.38-t / 2, \quad A_{24}=2.50-s / 2$ where $s, t \in \mathbb{R}$.

2 The parameters $A_{i j}$ corresponding to Fig. 8 are: $A_{11}=1+2 \sqrt{3} \tau, A_{22}=1-3 \sigma$ $-\sqrt{3} \tau, A_{33}=1+3 \sigma-\sqrt{3} \tau, A_{12}=-3.04 \sigma+1.23 \tau, A_{23}=-0.457 \sigma-3.25 \tau, A_{31}=2.59 \sigma$ $+2.02 \tau$. 
where $k_{\alpha}(\lambda)$ are complex-valued functions continuous at $\lambda_{j}=0$. Then

$$
\lim _{\lambda_{j} \rightarrow 0} \mathscr{L}(h(\lambda)) \Psi(\lambda)=\mathscr{L}[h(0)] \Psi(0) .
$$

Proof. We simply observe that $\mathscr{L}(\alpha) \Psi(\lambda)(\alpha \in \mathscr{G}(N, N-1))$ is an integral over a fixed contour, which is continuous at $\lambda_{j}=0$.

Now consider the representation formulas (4.5.8) and (4.5.9); these are written in a certain basis $\left\{\Theta(\chi) \mid \chi \subset \Omega_{N}\right\}$; actually, $\Theta(\chi)$ depends on $\lambda$.

Lemma 5.2.2. a) The physical sheet $\Psi(\lambda)$ may be identified with $\Theta(\phi)(\lambda)(\phi=$ empty set $)$. b) The limiting vectors $\Theta(\chi)=\lim _{\lambda_{j} \rightarrow 0} \Theta(\chi)(\lambda)$ exist and satisfy

(Here $\Psi=\Psi(0))$.

$$
\begin{aligned}
& \Theta(\phi)=\Psi, \\
& \Theta(\chi)=-\sum_{\substack{\psi \supset \chi \\
\psi \neq \Omega}} \mathscr{L}\left[\left(a\left(\begin{array}{cc}
\Omega-\psi & \psi \\
\phi & \phi
\end{array}\right)\right)\right] \Psi+(-)^{m} \Psi .
\end{aligned}
$$

Proof. a) From Lemma 5.1.7 and (4.5.8) and (4.5.9) we see that $\Psi$ is proportional to $\Theta(\phi)$; the identification is a matter of normalization. b) This follows from Lemma 5.2.1 and the formulae of 4.5.

Theorem 5.2.3. Let $h \in \mathbb{C}(\mathscr{G}(N, N-1))$ be as in Lemma 5.2.1, and let $\mathscr{L}(h)(\lambda)_{\chi, \psi}$ be the matrix elements of $\mathscr{L}(h)$ (for generic $\lambda$ ) in the $\Theta(\chi)$ basis, i.e.

$$
\mathscr{L}(h)[\Theta(\psi)(\lambda)]=\sum_{\chi \subset \Omega_{N}} \mathscr{L}(h)(\lambda)_{\chi, \psi} \Theta(\chi)(\lambda) .
$$

Suppose that $\mathscr{L}(h)_{\chi, \psi}=\lim _{\lambda_{j} \rightarrow 0} \mathscr{L}(h)(\lambda)_{x, \psi}$ exists. Then (5.2.4) also holds in in the limit $\lambda_{j} \rightarrow 0$ :

$$
\mathscr{L}(h) \Theta(\psi)=\sum_{\chi \supset \Omega_{\mathrm{N}}} \mathscr{L}(h)_{\chi, \psi} \Theta(\chi) .
$$

Proof. From the hypothesis and Lemmas 5.2.1 and 5.2.2, both sides are continuous functions of $\lambda$.

Note that the limits of the matrix elements (4.5.8) and (4.5.9) exist. Again writing $\mathscr{L}\left(a(\mathscr{S})_{\chi, \psi}\right)=u(\mathscr{S})_{\chi} v(\mathscr{S})_{\psi}$, we have

$$
\begin{aligned}
& u\left(\begin{array}{ll}
\varrho_{1} & \varrho_{4} \\
\varrho_{3} & \varrho_{2}
\end{array}\right)_{\chi}=(-)^{\left|x \Delta \varrho_{2}\right|}\left\{\delta_{\varrho_{4} \subset x<\Omega-\varrho_{3}}+(-)^{N+m} \delta_{\varrho_{3} \subset x \subset \Omega-\varrho_{4}}\right\} \\
& v\left(\begin{array}{ll}
\varrho_{1} & \varrho_{4} \\
\varrho_{3} & \varrho_{2}
\end{array}\right)_{\psi}=-(-)^{\left|\varrho_{4}\right|}\left\{\delta_{\varrho_{1} \subset \psi \subset \Omega-\varrho_{2}}(-)^{m}+\delta_{\varrho_{2} \subset \psi \subset \Omega-\varrho_{1}}\right\} .
\end{aligned}
$$

From now on we take $\lambda_{j}=0$. Let $V$ denote the vector space of germs at $B_{0}$ spanned by analytic continuations of $\Psi$. From Theorem 5.2.3 and 
the following remark it is clear that the vectors $\Theta(\chi) \operatorname{span} V$. Then (5.2.5), (5.2.6), and (5.2.7) will provide a representation of $\mathscr{G}(N, N-1)$ in $V$ once we have proved

Theorem 5.2.8. The vectors $\Theta(\chi), \chi \subset \Omega_{N}$, are linearly independent. Hence $\operatorname{dim} V=2^{N-1}$.

Before proving 5.2.8 we give a lemma which is interesting in itself. Note that it may be shown directly that the physical integral (5.1.1) is singular on all Landau curves $M(\chi)$; we omit details.

Lemma 5.2.9. Let $\mathscr{L}(a(\mathscr{S}))=u(\mathscr{S}) \otimes v(\mathscr{S})$, as usual. If $\Phi$ is any sheet of the function, then $(v(\mathscr{S}), \Phi)=0$ iff $M(\mathscr{S})$ is not singular on $\Phi$.

Proof. This is trivially true for generic $\lambda$. Now suppose $(v(\mathscr{S}), \Phi)=0$, with $\Phi=\sum c(\chi) \Theta(\chi)$. Since $v(\mathscr{S})$ is not identically zero, there is a $\psi \subset \Omega_{N}$ such that $(v(\mathscr{S})(\lambda), \Theta(\psi)(\lambda))=d(\lambda)$ do not vanish for $\lambda \rightarrow 0$. Then if

define

$$
\Phi(\lambda)=\sum c(\chi) \Theta(\chi)(\lambda),
$$

$$
\Xi(\lambda)=\Phi(\lambda)-d(\lambda)^{-1}(v(\mathscr{S})(\lambda), \Phi(\lambda)) \Theta(\psi)(\lambda) .
$$

We see that $(v(\mathscr{S})(\lambda), \Xi(\lambda))=0$ for all $\lambda$, and that $\Phi=\lim _{\lambda \rightarrow 0} \Xi(\lambda)$. Thus $M(\mathscr{S})$ is not singular on $\Xi(\lambda)$, and hence not on $\Phi$.

Now suppose $(v(\mathscr{S}), \Phi) \neq 0$, but that $M(\mathscr{S})$ is not singular on $\Phi$. Since $v(\mathscr{S})$ is singular on some sheet $\Xi$ we have $(v(\mathscr{S}), \Xi) \neq 0$, and hence there is a sheet $\Xi^{\prime}=a \Xi+\Phi$, with $a \neq 0$, such that $\left(v(\mathscr{S}), \Xi^{\prime}\right)=0$. But then $M(\mathscr{S})$ is not singular on $\Xi^{\prime}$; this is a contradiction.

Proof of Theorem 5.2.8 Let $\Phi=\sum c(\chi) \Theta(\chi)$ have $c\left(\chi_{0}\right) \neq 0$ for some $\chi_{0}$; we must show $\Phi \neq 0$. But

$$
\left(v\left(\begin{array}{cc}
\chi_{0} & \phi \\
\phi & \Omega-\chi_{0}
\end{array}\right), \Phi\right)=(-)^{m+1} c\left(\chi_{0}\right) \neq 0,
$$

hence $M\left(\begin{array}{cc}\chi_{0} & \phi \\ \phi & \Omega-\chi_{0}\end{array}\right)$ is singular on $\Phi$ and $\Phi \neq 0$.

This completes the derivation of the representation formulae for case 3. We note that the formula $\operatorname{dim} V=2^{N-1}$ was derived by Boyling [14] and also follows from the homology decomposition theorem of Fotiadi, Froissart Lascoux, and Pham [15] (the same remark applies in the dimension formula of 5.3). It is also interesting to observe that the formulae (5.2.6) and (5.2.7) satisfy the Cutkosky-Steinman rules, as expected, and that the discontinuity around the leading curve vanishes when $N=m+1$ (in which case the leading curve is a pole). Note, however, that Lemma 5.2.9 is valid even when the curve $M(\mathscr{S})$ is a pole; thus our methods enable us to determine exactly on which sheets the poles appear. 


\subsection{Specialization of Parameters - Case 2}

In this section we study the integral with second kind singularities but without renormalization [(2.5) with (5.1.2) and (5.1.4)] under the specialization $\lambda_{j}=0, j=1, \ldots N, \lambda_{N+1}=N-m$. Now in the previous section we were able simply to take the limits of the matrix elements (4.5.8) and (4.5.9); the essential step in the argument was that the basis vectors used in (4.5.8) and (4.5.9) were continuous (in $\lambda$ ) linear combinations of group elements applied to the physical sheet. This is no longer true in the case under consideration; thus we must rewrite the matrix elements in a more suitable basis before taking the limit.

For the moment we consider generic $\lambda$. Note first that for any $\alpha(\mathscr{S}) \in \mathscr{G}(N+1, N-1)$ we have $v(\mathscr{S})_{\phi}=0$ [see (4.5.9)]. This means that we obtain a representation $\mathscr{L}^{*}$ of $\mathscr{G}(N+1, N-1)$, equivalent to the representation derived in 4.5 , by defining

$$
\mathscr{L}^{*}(x)=P \mathscr{L}(x), \quad x \in \mathbb{C}(\mathscr{G}(N+1, N-1)),
$$

where $P$ is the projection $P \Theta(\chi)=\left[1-\delta_{\chi, \phi}\right] \Theta(\chi)$. That is, $\mathscr{L}^{*}$ is a representation in the subspace of $E(N+1)$ spanned by $\{\Theta(\chi) \mid \chi \subset\{1, \ldots N\}$, $\chi \neq \phi\}$. We will work with $\mathscr{L}^{*}$ from now on; this simplifies the following calculations.

Lemma 5.3.1. The physical sheet $\Psi(=\Psi(\lambda))$ may be identified with $\Theta\left(\Omega_{N+1}\right)$.

Proof. See proof of Lemma 5.2.2.

We must now define a new basis of the appropriate type. For $\chi \subset \Omega_{N+1}$, we write $\chi^{*}=\Omega_{N+1}-\chi$.

Definition 5.3.2. For $\eta \subset \Omega_{N+1}$, with $\eta \neq \phi$,

otherwise.

$$
\Phi(\eta)=\left\{\begin{array}{c}
\Theta(\eta)(=\Psi), \quad \text { if } \quad \eta=\Omega_{N+1} \\
\sum_{\substack{\chi \subset \eta \\
\chi \neq \phi}}(-)^{|\chi|+1} X\left(\chi^{*}\right)^{-1} \mathscr{L}^{*}\left[a\left(\begin{array}{cc}
\chi & \chi^{*} \\
\phi & N+1
\end{array}\right)\right] \Psi
\end{array}\right.
$$

Using (4.5.8) and (4.5.9) we can calculate the relationship between the bases $\{\Theta(\chi)\}$ and $\{\Phi(\chi)\}$, (where $\chi \subset \Omega_{N+1}, \chi \neq \phi$ ). Writing $\Phi(\eta)$ $=T_{\eta \chi} \Theta(\chi)$ gives

$$
\begin{gathered}
T_{\eta, \chi}=-\delta_{\eta, \chi^{*}}-(-)^{\left|\chi^{*}\right|} X(\chi) Y_{N+1} \delta_{\eta, \chi} \\
+\delta_{\chi, \Omega_{N+1}}+\delta_{\eta, \Omega_{N+1}} \delta_{\chi, \Omega_{N+1}} X(\Omega) \\
\left(T^{-1}\right)_{\chi, \eta}=\left(1+Y_{N+1}\right)^{-1}\left\{-\delta_{\chi, \eta^{*}}+(-)^{|x|} X\left(\chi^{*}\right) Y_{N+1} \delta_{\chi, \eta}\right. \\
\left.+\left[1-(-)^{|x|} X\left(\chi^{*}\right) Y_{N+1}\right] \delta_{\eta, \Omega_{N+1}}-Y_{N+1} \delta_{\eta, \Omega_{N+1}} \delta_{\chi, \Omega_{N+1}}\right\}
\end{gathered}
$$


Note that the factor $\left(1+Y_{N+1}\right)^{-1}$ in (5.3.4) is singular for $\lambda_{N+1} \rightarrow N-m$. This shows that the $\Theta$ basis is indeed not appropriate for the specialization.

As before we write $\mathscr{L}^{*}(\mathscr{S})=u(\mathscr{S}) \otimes v(\mathscr{S})$. We may derive the components of $u$ and $v$ in the $\Phi$ basis, which we denote by primes, from (4.5.8), (4.5.9), (5.3.3), and (5.3.4).

a) First kind singularities:

$$
\begin{aligned}
& u^{\prime}\left(\begin{array}{cc}
\mu_{1} & \mu_{4} \\
\mu_{3} & \mu_{2} \cup\{N+1\}
\end{array}\right)_{\eta} \\
& =-\delta_{\mu_{3} \subset \eta \subset \mu_{4}}(-)^{\left|\eta^{*} \cup \mu_{2}\right|} \lambda\left(\eta^{*} \cap \mu_{2}\right)\left\{1-\delta_{\eta, \Omega_{N+1}} X(\Omega)\right\},
\end{aligned}
$$

$$
\begin{aligned}
v^{\prime}\left(\begin{array}{cc}
\mu_{1} & \mu_{4} \\
\mu_{3} & \mu_{2} \cup\{
\end{array}\right) & \{N+1\})_{\eta} \\
= & -(-)^{\left|\mu_{2}\right|} \lambda^{-1}\left(\mu_{2}\right)\left\{\delta_{\mu_{2} \subset \eta \subset \mu_{1}^{*}}(-)^{\left|\eta^{*} \cup \mu_{4}\right|} X\left(\eta \cup \mu_{4}\right)\right. \\
& -\delta_{\mu_{1} \subset \eta \subset \mu_{2}^{*}}(-)^{\left|\mu_{4}-\eta\right|} \lambda\left(\eta \cap \mu_{4}\right) \\
& \left.-\delta_{\mu_{2}, \phi}(-)^{N} X\left(\mu_{4}\right)\left[1+\delta_{\eta, \Omega_{N+1}} X(\Omega)\right]\right\} .
\end{aligned}
$$

b) Second kind singularities:

$$
\begin{aligned}
& u^{\prime}\left(\begin{array}{cc}
\mu_{1} & \mu_{4} \cup\{N+1\} \\
\mu_{3} & \mu_{2}
\end{array}\right)_{\eta} \\
& =-\left[1-\delta_{\eta, \Omega_{N}+1} X(\Omega)\right]\left\{\delta_{\mu_{3} \subset \eta \subset \mu^{4}}(-)^{N+\left|\eta-\mu_{2}\right|} \lambda\left(\mu_{2}-\eta\right)\right. \\
& \left.+\delta_{\mu_{4} \subset \eta \subset \mu_{3}^{\star}}(-)^{\left|\eta^{*} \cap \mu_{2}\right|} X\left(\eta^{*} \cup \mu_{2}\right)\right\} \text {, } \\
& v^{\prime}\left(\begin{array}{cc}
\mu_{1} & \mu_{4} \cup\{N+1\} \\
\mu_{3} & \mu_{2}
\end{array}\right)_{\eta} \\
& =-(-)^{\left|\mu_{2}\right|} \lambda^{-1}\left(\mu_{2}\right)\left\{\delta_{\mu_{2} \subset \eta \subset \mu_{1}^{*}}(-)^{\left|\eta^{*} \cup \mu_{4}\right|} Y_{N+1} X\left(\eta \cup \mu_{4}\right)\right\} \text {. }
\end{aligned}
$$

We may now argue as in 5.2. In particular, the basis vectors $\Phi(\eta)$ have limits when we specialize the $\lambda$ 's given by

$$
\Phi(\eta)=\left\{\begin{array}{l}
\Psi, \quad \text { if } \quad \eta=\Omega_{N+1} \\
\sum_{\substack{\chi \subset \eta \\
\chi \neq \phi}}(-)^{m} \mathscr{L}^{*}\left[a\left(\begin{array}{cc}
\chi & \chi^{*} \\
\phi & N^{+} 1
\end{array}\right)\right] \Psi
\end{array}\right.
$$

otherwise. 
The equivalent of Theorem 5.2.3 holds; the limiting "matrix elements" $\mathscr{L}^{*}(h)_{\chi, \psi}$ may be obtained from (5.3.5)-(5.3.8). Thus

a) First kind singularities:

$$
\begin{gathered}
u^{\prime}\left(\begin{array}{cc}
\mu_{1} & \mu_{4} \\
\mu_{3} & \mu_{2} \cup\{N+1\}
\end{array}\right)_{\eta} \\
=-\delta_{\mu_{3} \subset \eta \subset \mu_{4}^{*}}(-)^{\left|\eta^{*} \Delta \mu_{2}\right|}\left\{1-(-)^{m} \delta_{\eta, \Omega_{N+1}}\right\} \\
v^{\prime}\left(\begin{array}{cc}
\mu_{1} & \mu_{4} \\
\mu_{3} & \mu_{2} \cup\{N+1\}
\end{array}\right)_{\eta} \\
\quad=(-)^{\left|\mu_{4}\right|}\left\{\delta_{\mu_{2} \subset \eta \subset \mu_{3}^{*}}(-)^{m}+\delta_{\mu_{3} \subset \eta \subset \mu_{2}^{*}}\right. \\
\left.-\delta_{\mu_{2}, \phi}(-)^{m}\left[1+(-)^{m} \delta_{\eta, \Omega_{N+1}}\right]\right\} .
\end{gathered}
$$

b) Second kind singularities:

$$
\begin{gathered}
u^{\prime}\left(\begin{array}{cc}
\mu_{1} & \mu_{4} \cup\{N+1\} \\
\mu_{3} & \mu_{2}
\end{array}\right)_{\eta} \\
=-\left(1-(-)^{m} \delta_{\eta, \Omega_{N+1}}\right)\left\{\delta_{\mu_{3} \subset \eta \subset \mu_{4}^{*}}(-)^{N+\left|\eta \Delta \mu_{2}\right|}\right. \\
+\delta_{\mu_{4} \subset \eta \subset \mu_{3}^{*}}(-)^{\left.m+1+\left|\eta \Delta \mu_{2}\right|\right\},} \\
v^{\prime}\left(\begin{array}{cc}
\mu_{1} & \mu_{4} \cup\{N+1\} \\
\mu_{3} & \mu_{2}
\end{array}\right)_{\eta}=-\delta_{\mu_{2} \subset \eta \subset \mu_{1}^{*}}(-)^{m+\left|\mu_{4}\right|} .
\end{gathered}
$$

We finally have

Theorem 5.3.14. The vectors $\left\{\Phi(\eta) \mid \eta \subset \Omega_{N+1}, \eta \neq \Phi\right\}$, given by (5.3.9), are linearly independent. Hence the vector space $V$ has dimension $2^{N}-1$, and (5.3.10)-(5.3.13) give the representation of $\mathscr{G}(N+1, N-1)$ in $V$.

Proof. We argue as in the proof of Theorem 5.2.8 (Lemma 5.2.4 applies unchanged); using the dual vectors

$$
\left\{v\left(\begin{array}{cc}
\chi & \{N+1\} \\
\phi & \Omega_{N+1}-\chi
\end{array}\right)\left|\chi \subset \Omega_{N+1}, 1 \leqq\right| \chi \mid \leqq N-1\right\}
$$

and $v\left(\begin{array}{cc}\Omega_{N+1} & \phi \\ \phi & \{N+1\}\end{array}\right)$.

We note from (5.3.12) that the leading second kind singularity is a pole whenever $N+m$ is even, as expected.

\subsection{Renormalization (Case 1)}

In this section we study the integral (2.2) in the case $N<\frac{m}{2}$, where $m$ is even, using the method of analytic renormalization [3]. Let $\mathscr{J}$ be a 
generalized evaluator in the sense of [3]; as in [1], we require that $\mathscr{J}$ satisfy an additional property as follows: Let $f\left(\lambda_{1}, \ldots \lambda_{N}\right)$ be any function in the domain of $\mathscr{J}$, and, for $z \in \mathbb{C}$, define $f_{z}\left(\lambda_{1}, \ldots \lambda_{N}\right)=f\left(z \lambda_{1}, \ldots z \lambda_{N}\right)$. Then $\mathscr{J}$ must satisfy

$$
\mathscr{J} f_{z}=\mathscr{J} f \text {. }
$$

The fundamental result of the theory of analytic renormalization may be stated, for our case, as follows. Define

$$
E(\lambda, A)=\Gamma(\mu) \int_{\alpha_{i} \geqq 0} \frac{\prod_{i=1}^{N} \alpha_{i}^{\lambda_{i}}\left(-\sum_{1}^{N} \alpha_{i}\right)^{N-m}}{D(\alpha, A)^{\mu}} \eta(\alpha),
$$

where $\mu=N-\frac{m}{2}+\frac{1}{2}\left(\sum_{1}^{N} \lambda_{i}\right)$. (Note that this is the generic integral (2.5) with (5.1.2), (5.1.4), the additional specialization $\lambda_{N+1}=N-m$, and a factor $\Gamma(\mu)$.) Then the physical sheet of the renormalized integral is given by

$$
F(A)=\mathscr{J} E(\lambda, A) .
$$

We wish to study the monodromy representation $\mathscr{L}_{\mathscr{g}}$ of $F(A) . F(A)$ is analytic in the same region (in $A$ ) as $E(\lambda, A)$; that is, the appropriate fundamental group is $\mathscr{G}(N+1, N-1)$ as in 5.3. Since the physical sheet is the same, we use the representation formulae (5.3.5)-(5.3.9) for $E(\lambda, A)$. We will adapt the notation of 5.3, writing, for $\eta \subset \Omega_{N+1}, \eta \neq \phi$,

$$
\Phi(\eta)=\left\{\begin{array}{c}
E(\lambda, A)(=\Psi) \text { if } \eta=\Omega_{N+1} \\
\sum_{\chi \subset \eta}(-)^{|x|+1} X\left(\chi^{*}\right)^{-1} \mathscr{L} *\left[a\left(\begin{array}{cc}
\chi & \chi^{*} \\
\phi & N+1
\end{array}\right)\right] \Psi
\end{array}\right.
$$

otherwise.

We now review briefly the basic techniques for obtaining the monodromy of renormalized integrals; a fuller discussion is given in [1]. These are based on the important remark that evaluation by $\mathscr{J}$ commutes with analytic continuation in the variables $A_{i j}$ : for any $\alpha \in \mathscr{G}(N+1, N-1)$ and any $\eta \subset \Omega_{N+1}, \eta \neq \phi$, we have

$$
\mathscr{J}\left[\mathscr{L}^{*}(\alpha) \Phi(\eta)\right]=\mathscr{L}_{\mathscr{J}}(\alpha)[\mathscr{J} \Phi(\eta)] .
$$

Now for any discontinuity operator $a=a(\mathscr{S})$ we obtain from 5.3 a formula

$$
\mathscr{L}^{*}(a) \Phi(\eta)=\sum_{\chi}\left[\mathscr{L}^{*}(a)\right]_{\eta, \chi} \Phi(\chi),
$$

where the matrix elements $\mathscr{L}_{\eta, \chi}^{*}$ depend on $\lambda$, and are analytic at the point $\lambda_{i}=0,1 \leqq i \leqq N$. If we expand these matrix elements in Taylor 
series, apply $\mathscr{J}$ to (5.4.5), and use (5.4.4) we obtain an expression for $\mathscr{L}_{\mathscr{J}}(a)[\mathscr{J} \Phi(\eta)]$ as a (possibly infinite) linear combination of functions $\mathscr{J}[M \Phi(\eta)]$, where $M$ is a monomial in the $\lambda_{i}$ 's. By multiplying (5.4.5) by $M$ before applying $\mathscr{J}$ we obtain a similar expression for $\mathscr{L}_{\mathscr{J}}[\mathscr{J} M \Phi(\eta)]$. Thus to determine the representation $\mathscr{L}_{\mathscr{g}}$ we need only find the linear relations between the functions $\mathscr{J}[M \Phi(\eta)]$.

Lemma 5.4.6. Let $M=\prod_{i=1}^{N} \lambda_{i}^{m_{\imath}}\left(m_{i} \geqq 0\right)$ be any monomial, and take $\eta \subset \Omega_{N+1}, \eta \neq \phi$. Then

a) $\mathscr{J}[M \Phi(\eta)]=0$, if $\sum_{1}^{N} m_{i}>1$;

b) $\mathscr{J}\left[\lambda_{k} \Phi\left(\Omega_{N+1}\right)\right]=P(A)$, where $P$ is a polynomial in $\left\{A_{i j}\right\}$. which is independent of $k$;

c) $\mathscr{J}\left[\lambda_{k} \Phi(\eta)\right]=0$, if $\eta \neq \Omega_{N+1}$.

Proof. From (5.4.0) and the symmetry property of $\mathscr{J}$ we have

$$
\mathscr{J}\left[M\left(\sum_{i=1}^{N} \lambda_{i}\right)^{-1}\right]= \begin{cases}N^{-1}, & \text { if, for some } k, m_{i}=\delta_{i k} ; \\ 0 & \text { otherwise. }\end{cases}
$$

Then a) and b) follow directly from (5.4.1) (we observe that any $\Phi(\eta)$ is analytic at $\lambda_{i}=0$ except for the factor $\left.\Gamma(\mu)\right)$. From b) we have, for any $a=a(\mathscr{S})$,

$$
\mathscr{J}\left[\lambda_{k} \mathscr{L}^{*}(a) \Psi\right]=\mathscr{L}_{\mathscr{J}}\left[\mathscr{J} \lambda_{k} \Psi\right]=0 ;
$$

this, combined with (5.4.3), proves c).

We thus see that the determinations of $F(A)$ span a finite-dimensional vector space $V_{\mathscr{J}}$ spanned by the $2^{N}$ vectors

$$
\Xi(\eta)=\left\{\begin{array}{lll}
\mathscr{J} \Phi(\eta) & \text { if } & \eta \subset \Omega_{N+1}, \eta \neq \phi \\
\mathscr{J}\left[\lambda_{k} \Psi\right] & \text { if } & \eta=\Phi
\end{array}\right.
$$

We have, arguing as above, that

$$
\mathscr{L}_{\mathscr{J}}(a(\mathscr{S})) \Xi(\eta)=\sum_{\chi \subset \Omega_{N+1}} v_{\mathscr{S}}(\mathscr{S})_{\eta} u_{\mathscr{J}}(\mathscr{S})_{\chi} \Xi(\chi),
$$

where $v_{\mathscr{F}}(\mathscr{S})_{\eta}$ and $u_{\mathscr{F}}(\mathscr{S})_{\chi}$ are given by (5.3.10)-(5.3.13) for $\eta, \chi \neq \phi$ and, for $\eta$ or $\chi=\phi$, by

$$
\begin{gathered}
u_{\mathscr{I}}\left(\begin{array}{cc}
\mu_{1} & \mu_{4} \\
\mu_{3} & \mu_{2} \cup\{N+1\}
\end{array}\right)_{\phi}=N \pi i(-)^{\left|\mu_{2}\right|} \delta_{\mu_{4}, \phi}, \\
u_{\mathscr{J}}\left(\begin{array}{cc}
\mu_{1} & \mu_{4} \cup\{N+1\} \\
\mu_{3} & \mu_{2}
\end{array}\right)_{\phi}=N \pi i(-)^{\left|\mu_{2}\right|}\left\{\delta_{\mu_{4}, \phi}-(-)^{N} \delta_{\mu_{3}, \phi}\right\}, \\
v_{\mathscr{J}}(\mathscr{S})_{\phi}=0 \text { for all } \mathscr{S} .
\end{gathered}
$$


An argument similar to that of 5.3 shows that all vectors $\left\{\Xi(\eta) \mid \eta \subset \Omega_{N+1}\right\}$ are linearly independent; thus the dimension of $V_{\mathscr{g}}$ is $2^{N}$, and (5.3.10)(5.3.13) with (5.4.7)-(5.4.9) give $\mathscr{L}_{\mathscr{J}}$.

\section{§ 6. The Case 4 in which the Invariants are not Algebraically Independent}

\subsection{The Transformation of the Integral}

In this section we show how in the case $N \geqq m+2$ the momentum space integral (2.2) may be represented as a specialization of the generic integral (2.5).

We begin by making the inversion compactification of momentum space [15]. Embed $\mathbb{C}^{m}$ in $\mathbb{P}^{m+1}$ by mapping

$$
k \in \mathbb{C}^{m} \text { into }\left(k^{2}, k, 1\right)=\left(z_{0}, z, z_{m+1}\right) \in \mathbb{P}^{m+1}
$$

where $z_{i} 0 \leqq i \leqq m+1$ denote the coordinates of a point in $\mathbb{P}^{m+1}$. The transformed integral may be written

$$
\int \delta\left(B_{1}(z)\right) \prod_{j=1}^{N}\left[l_{j}(z)\right]^{-1} \stackrel{m+1}{\eta}(z)\left(z_{0}\right)^{N-m}
$$

where

$$
\begin{gathered}
B_{1}(z) \equiv\left(z_{m+1}\right)\left(z_{0}\right)-\sum_{i=1}^{m} z_{i}^{2} \\
l_{j}(z) \equiv\left(k_{j}^{2}+m_{j}^{2}\right) z_{m+1}+2 z \cdot k_{j}+z_{0} \text { for } 1 \leqq j \leqq N .
\end{gathered}
$$

(6.1.1) bears an evident relation to the generic integral (2.5) for $r=N$, $s=m+1$ which will be elaborated below.

We denote by $T=\mathbb{C}^{(s+1) N}$ the space of the coefficients of the linear forms $l_{j}$ in (2.5), and by $W=\mathbb{C}^{(s+1)(s+2) / 2}$ the space of the coefficients of the quadratic form $B$. Let $t_{0} \in T$ be a point such as discussed in Remark 3.4.4 $(C)$, i.e. $l_{j i}=\zeta_{j}^{k(i)}$, where $k(0)=1, k(i)<k(i+1)$, and $0<\zeta_{1} \ll \zeta_{2} \ll \cdots \ll \zeta_{N}$. For $\chi \subset \Omega$ with $|\chi| \leqq s$ we define

$$
\begin{aligned}
L(\chi)= & \left\{(l, B): \text { the intersection }\left\{z \in \mathbb{P}^{s}: B(z)=0 l_{j}(z)=0 j \in \chi\right\}\right. \\
& \text { is singular }\} \subset T \times W
\end{aligned}
$$

and let $L=\cup L(\chi)$. From (6.1.3) it is evident that any path with base point $\left\{k_{j}=\frac{1}{2}\left(\zeta_{j}, \ldots, \zeta_{j}^{m}\right) m_{j}^{2}=\zeta_{j}^{(m+1)}-\frac{1}{4} \sum_{k=1}^{m} \zeta_{j}^{2 k}\right\}$ in the space of the variables $k_{j}, m_{j}$ may be considered as a path in $T$ with base point $t_{0}$. (Note that by suitable choice of $\zeta_{i}$ and $k(i)$ we may arrange to have $m_{j}^{2}>0$ for all $j$.) $4^{*}$ 
Therefore our problem is to determine the action of $\pi_{1}\left(T \times\left\{B_{1}\right\}-L\right.$; $\left.\left(t_{0}, B_{1}\right)\right)$ on $V(N, s)$, the vector space of germs of the integral. We note that the integral (6.1.1) may be extended to a function on $T \times W$ by allowing the coefficients of the $l_{j}$ to vary in $T$ and replacing $B_{1}$ by an arbitrary $B$. We will show that the action of any element of $\pi_{1}\left(T \times\left\{B_{1}\right\}-L\right.$; $\left.\left(t_{0}, B_{1}\right)\right)$ may be realized also as the action of a suitable element of $\pi_{1}\left(\left\{t_{0}\right\} \times W-L ;\left(t_{0}, B_{1}\right)\right)$, i.e. by varying the coefficients of $B$ while keeping the coefficients of the $l_{j}$ fixed. The action of $\pi_{1}\left(\left\{t_{0}\right\} \times W-L\right.$; $\left.\left(t_{0}, B_{1}\right)\right)$ may be deduced from the results of $\S 4$ on the generic integral.

Theorem 6.1.4. The natural map

is onto.

$$
\pi_{1}\left(\left\{t_{0}\right\} \times W-L ;\left(t_{0}, B_{1}\right)\right) \rightarrow \pi_{1}\left(T \times W-L ;\left(t_{0}, B_{1}\right)\right)
$$

Proof. This theorem is an immediate application of Lemma 6.1.5 below since the irreducible components $L(\chi)$ of $L$ are given by polynomial equations of positive degree in $B$.

Let $T_{1}=\mathbb{C}^{n_{1}}, T_{2}=\mathbb{C}^{n_{2}}$ be two spaces of $n_{1}, n_{2}$ complex variables respectively. We consider an algebraic variety $N$,

$$
N \subset T_{1} \times T_{2},
$$

given as a union of irreducible components $N_{\alpha}$,

$$
N_{\alpha}=\left\{\left(t_{1}, t_{2}\right): f_{\alpha}\left(t_{1}, t_{2}\right)=0\right\},
$$

where $f_{\alpha}$ is a polynomial in $t_{1}, t_{2}$ of positive degree in $t_{1}$.

Lemma 6.1.5. Let $\left(t_{10}, t_{20}\right)$ be a generic point in $T_{1} \times T_{2}$. Then the natural injection

$$
i_{*}: \pi_{1}\left(T_{1} \times\left\{t_{20}\right\}-N ;\left(t_{10}, t_{20}\right)\right) \rightarrow \pi_{1}\left(T_{1} \times T_{2}-N ;\left(t_{10}, t_{20}\right)\right)
$$

is onto.

Proof. Let $\bar{T}_{1}=\mathbb{P}^{n_{1}}$ denote the projective compactification of $T_{1}$, and $T_{\infty}=\bar{T}_{1}-T_{1}$ the hyperplane at infinity in this space. We have trivially a commutative diagram

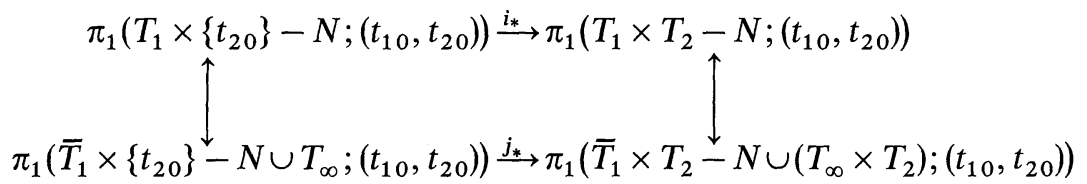

where the vertical maps are isomorphisms. The assertion of the lemma is thus equivalent to the assertion that $j_{*}$ is onto. 
To prove that $j_{*}$ is onto we make use of Hironaka's theory of disingularization ([16], cf. also [17]). According to this theory we may find a pair $Y, S$ together with a map $p: Y \rightarrow T_{2}$ such that

a) $Y$ is an algebraic manifold of complex dimension $n_{1}+n_{2}$,

b) $S$ is an algebraic subvariety of $Y$ in general position in $Y$,

c) there is a biholomorphic map

$$
f: Y-S \rightarrow \bar{T}_{1} \times T_{2}-N \cup\left(T_{\infty} \times T_{2}\right),
$$

d) $p$ is a proper surjective algebraic map such that on $Y-S$

$$
p=q f
$$

where $q: \bar{T}_{1} \times T_{2} \rightarrow T_{2}$ is the natural projection. Note that from c) and d) we obtain isomorphisms $f_{*}, f_{*}^{\prime}$ such that the following diagram commutes

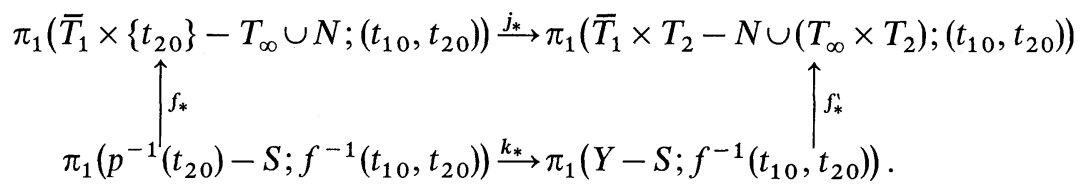

Our object is now to prove that $k_{*}$ is onto.

Denote by $A \subset T_{2}$ the algebraic subvariety of $T_{2}$ defined by

$$
A=\left\{\left(t_{2}\right): S \cap p^{-1}\left(t_{2}\right) \text { is not in general position in } p^{-1}\left(t_{2}\right)\right\} .
$$

We construct by means of the shadow method ([18], p. 32) a cut $C$ bounding on $A$ in $T_{2}$ such that $T_{2}-C$ is simply connected and contains $t_{20}$. Set $B=p^{-1}(A), D=p^{-1}(C) \subset Y$. Our assertion that $k_{*}$ is onto will follow from

i) the fact that the natural injection

$$
\pi_{1}\left(Y-S \cup D ; f^{-1}\left(t_{10}, t_{20}\right)\right) \rightarrow \pi_{1}\left(Y-S ; f^{-1}\left(t_{10}, t_{20}\right)\right)
$$

is onto, together with

ii) the isomorphism

$$
\pi_{1}\left(Y-S \cup D ; f^{-1}\left(t_{10}, t_{20}\right)\right) \leftrightarrow \pi_{1}\left(p^{-1}\left(t_{20}\right)-S ; f^{-1}\left(t_{10}, t_{20}\right)\right) .
$$

The isomorphism is an immediate consequence of the ambiant isotopy theorem ([15]) which implies the triviality of the fibre space $Y-S \cup D, p$ over $T_{2}-C$. For (i) we must show that any $\alpha \in \pi_{1}(Y-S$; $\left.f^{-1}\left(t_{10}, t_{20}\right)\right)$ has a representative $\underline{\alpha}$ which does not intersect $D$. To construct $\underline{\alpha}$ take any representative $\underline{\alpha}^{\prime}$ of $\alpha$. By modifying $\underline{\alpha}^{\prime}$ infinitesimally if necessary we can arrange that $\underline{\alpha}^{\prime}$ intersects $D$ transversely in a finite number of points. Now

$$
D \cap S=\bigcup_{t_{2} \in \boldsymbol{B}}\left(p^{-1}\left(t_{2}\right) \cap S\right)
$$




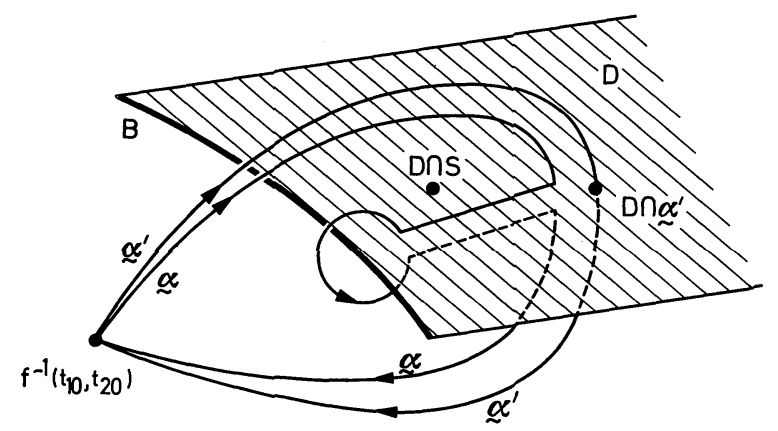

Fig. 9. The construction of the cut

is of codimension two in $D$, since our condition that $f_{\alpha}$ have positive degree in $t_{1}$ for all $\alpha$ implies that $p^{-1}\left(t_{2}\right) \cap S$ is of codimension two in $p^{-1}\left(t_{2}\right)$ for all $t_{2}$ not in some subset of codimension $\geqq 4$ in $T_{2}$. This means that $D-D \cap S$ is connected and for each point of $\underline{\alpha}_{1}^{\prime} \cap D$ we may construct a path in $D$ to a point of $B . \underline{\alpha}$ is then constructed in the way indicated in Fig. 9 which illustrates the case in which there is one intersection. This completes the proof of the lemma and hence of Theorem 6.1.4.

Theorem 6.1.4 reduces our problem to the study of (6.1.1) for variable $B$ and fixed $\left\{l_{j}\right\}=t_{0}$. Our fundamental group is then $\pi_{1}\left(\left\{t_{0}\right\} \times W-L\right.$; $\left.\left(t_{0}, B_{1}\right)\right)$. The general discussion in $\S 3$ gives us a presentation of $\pi_{1}\left(\left\{t_{0}\right\} \times W-L ;\left(t_{0}, B_{0}\right)\right)$ where $B_{0}$ is positive definite. We identify these groups by means of the isomorphism induced by conjugation with the standard path from $B_{0}$ to $B_{1}$. Our fundamental group is thus $\mathscr{G}(N, s)$ $(s=m+1)$.

To write (6.1.1) as a specialization of the generic integral (2.5) we use the residue theorem of Leray to replace $\delta(B(z))$ by $[B(z)]^{-1}$ with a corresponding change of the contour of integration to a coboundary. The resulting integral is a specialization of (2.5) with

$$
\lambda_{i}=-1 \quad 1 \leqq i \leqq N \quad \mu=1 .
$$

The construction of the monodromy representation of $\mathscr{G}(N, s), \mathscr{L}(\lambda)$, is obtained as follows: First we embed $\mathscr{G}(N, s)$ into $\mathscr{G}(N)$ by means of the homomorphism

$$
i_{R}: \alpha(\mathscr{S}) \rightarrow \alpha\left(\mathscr{S}^{R}\right)
$$

where $\mathscr{S}^{R}$ is the signature obtained from $\mathscr{S}$ by rotating the subsets $\varrho_{1}, \varrho_{2}$, $\varrho_{3}, \varrho_{4}$ clockwise (i.e. $\varrho_{1}^{R}=\varrho_{3}$ etc.). Then we take the generic representation of $\mathscr{G}(N)$ and restrict it to $i_{R} \mathscr{G}(N, s) \subset \mathscr{G}(N)$. There will be no confusion if in what follows we identify $i_{R} \mathscr{G}(N, s)$ with $\mathscr{G}(N, s)$. The use of $i_{R}$ rather than the evident homomorphism $i: \alpha(\mathscr{S}) \rightarrow \alpha(\mathscr{S})$ discussed in 4.5 is 
purely a matter of convenience to avoid the necessity for a change of the basis in which we have given the representation of $\mathscr{G}(N) . \mathscr{G}(N, s) \subset \mathscr{G}(N)$ is with this convention the subgroup generated by those signatures $\mathscr{S}$ which contain a reduced signature $\mathscr{T}_{\chi} \chi \subset \Omega,|\chi|=s$ of the form

$$
\mathscr{T}_{\chi}=\left(\begin{array}{cc}
\phi & \{j \in \chi:|\chi \cap(j, N]| \text { even }\} \\
\{j \in \chi:|\chi \cap(j, N]| \text { odd }\} & \phi
\end{array}\right) .
$$

This condition is determined by the choice of $t_{0}$ (Remark 3.4.4 (C)). The constants $X(\chi)$ are given by

$$
X(\chi)=(-1)^{m+1+N+|\chi|} \exp 2 \pi i\left(\sum_{i \notin \chi} \lambda_{i}-\mu\right) .
$$

Note that a signature $\mathscr{S}=\left(\begin{array}{ll}\varrho_{1} & \varrho_{4} \\ \varrho_{3} & \varrho_{2}\end{array}\right)$ corresponding to a point of the Landau variety $L(\chi)$ will have $\chi=\varrho_{1} \cup \varrho_{2}$.

The physical sheet is obtained by taking as contour of integration in (6.1.1) the real section of $\{B(z)=0\}$, and the corresponding contour in the generic integral. Comparison with Appendix A shows that when this contour is deformed to give the analytic continuation along the standard path from $B_{1}$ to $B_{0}$ it becomes the contour $\gamma_{0}$ of Definition A.7. Then from Theorem A.2 we obtain the physical sheet conditions

$$
a\left(\begin{array}{ll}
\varrho_{1} & \varrho_{4} \\
\varrho_{3} & \varrho_{2}
\end{array}\right) \Psi=0 \quad \text { unless } \quad \varrho_{1} \text { or } \varrho_{2}=\phi
$$

(in this equation we have already taken into account the rotation of the signature). In the space of the generic representation these conditions uniquely characterize $\Psi$ and enable us to make the identification

$$
\Psi=\Theta(\phi)
$$

\subsection{Specialization of Parameters - Case 4}

The process of specializing the $\lambda$ 's in the case of non-independent invariants is made somewhat difficult by the rather complicated restrictions on admissibility of signatures which arise from the positional characteristic. To study this process we need to give some combinatorial definitions and results. Proofs of the results will be omitted, they are straightforward although tedious. The reader is advised, however, to follow the discussion by applying it to a special case (say $N=6, m=3$ ). 
Definition 6.2.1. For any $\chi \subset \Omega$, define $\varrho_{i}(\chi), i=1, \ldots 4$, by

$$
\begin{aligned}
& \varrho_{1}(\chi)=\{j \in \chi|| \chi \cap(j, N] \mid \text { even }\} \\
& \varrho_{2}(\chi)=\{j \in \chi|| \chi \cap(j, N] \mid \text { odd }\} \\
& \varrho_{3}(\chi)=\{j \notin \chi|| \chi \cap(j, N] \mid \text { odd }\} \\
& \varrho_{4}(\chi)=\{j \notin \chi|| \chi \cap(j, N] \mid \text { even }\} .
\end{aligned}
$$

Here, as usual, $(j, N]=\{j+1, \ldots N\} \quad((N, N]=\phi)$; compare with Remark 3.4.4 (C).

Definition 6.2.2. A block $b \subset \Omega$ is a nonempty sequence of consecutive integers. The block sequence for a set $\psi \subset \Omega$ is the (unique) sequence $b_{1}, \ldots b_{k}$ of blocks such that

$$
1 \in b_{1}, \quad \max \left\{j \in b_{i}\right\}=\min \left\{j \in b_{i+1}\right\}-1,
$$

for all $i=1, \ldots k-1$, and $\psi=b_{k} \cup b_{k-2} \cup \ldots$. We write $b(\psi)=k$.

Definition 6.2.3. We wish to define two order relations written $<$ and $\prec$, on the subsets of $\Omega$.

a) We write $\chi_{1}<\chi_{2}$ if either $\left|\chi_{1}\right|<\left|\chi_{2}\right|$ or $\left|\chi_{1}\right|=\left|\chi_{2}\right|$ and $\chi_{1}$ preceeds $\chi_{2}$ lexicographically when written as a sequence of increasing integers.

b) For $\psi \subset \Omega$, let $b_{1}, \ldots b_{k}$ be the block sequence for $\psi$, let $j_{i}$ be the smallest element of $b_{i}, i=1, \ldots k$, and write $\eta(\psi)=\left\{j_{1}, \ldots j_{k}\right\}$. Then $\psi_{1} \prec \psi_{2}$ iff $\eta\left(\psi_{1}\right)<\eta\left(\psi_{2}\right)$ (in the sense of a) above).

Definition 6.2.4. For $\chi \subset \Omega_{N}$ (i.e., $N \notin \chi$ ), define $\mu(\chi)=\varrho_{1}(\chi) \cup \varrho_{3}(\chi)$.

Lemma 6.2.5. a) for $\chi \subset \Omega_{N}, b[\mu(\chi)]=|\chi|$.

b) If $\chi_{1}, \chi_{2} \subset \Omega_{N}$, then $\chi_{1}<\chi_{2}$ implies $\mu\left(\chi_{1}\right) \prec \mu\left(\chi_{2}\right)$.

c) $\left|\left\{\psi \subset \Omega_{N} \mid b(\psi)=s\right\}\right|=\left(\begin{array}{c}N-1 \\ s\end{array}\right)$.

Corollary 6.2.6. The mapping $\chi \rightarrow \mu(\chi)$ is a bijective correspondence between $\left\{\chi \subset \Omega_{N}|| \chi \mid=s\right\}$ and $\left\{\psi \subset \Omega_{N} \mid b(\psi)=s\right\}$.

Definition 6.2.7. We define $\mathscr{A}(N, s)=\left\{\psi \subset \Omega_{N} \mid b(\psi) \leqq s\right\}$.

Theorem 6.2.8. Take $\chi \subset \Omega_{N}$. Then, for all values of the parameters $\lambda$, we have

a) $u\left(\begin{array}{cc}\chi & \varrho_{4}(\chi) \\ \varrho_{3}(\chi) & \phi\end{array}\right)_{\mu(\chi)} \neq 0$

b) $u\left(\begin{array}{cc}\chi & \varrho_{4}(\chi) \\ \varrho_{3}(\chi) & \phi\end{array}\right)_{\psi}=0 \quad$ unless $\quad \psi \in \mathscr{A}(N,|\chi|)$ and $\psi \leqq \mu(\chi)$,

where $u$ is given by (4.5.8). (Recall that we use as basis for the generic representation $\left\{\Theta(\psi) \mid \psi \subset \Omega_{N}\right\}$.) 
We now turn to the specialization of the parameters $\lambda$. As in $\S 5$, we must work in a co-ordinate system in which the basis vectors have limits under the specialization.

Definition 6.2.9. For any $\chi \subset \Omega_{N}$ with $|\chi| \leqq s$ define

$$
\Xi(\chi)=\left\{\begin{array}{l}
\Psi \text { if } \chi=\phi \\
-\mathscr{L}\left[a\left(\begin{array}{cc}
\chi & \varrho_{4}(\chi) \\
\varrho_{3}(\chi) & \phi
\end{array}\right)\right] \Psi \quad \text { if } \quad \chi \neq \phi .
\end{array}\right.
$$

Remark 6.2.10. The limiting vectors $\lim _{\lambda_{j} \rightarrow-1} \Xi(\chi)$ exist.

Lemma 6.2.11. For any $\psi \subset \Omega_{N}, b(\psi) \leqq s$, the limiting vectors

exist.

$$
\lim _{\lambda_{j} \rightarrow-1} \Theta(\psi)
$$

Proof. We note first that the vector $\Xi(\chi)$ is proportional to $u\left(\begin{array}{cc}\chi & \varrho_{4}(\chi) \\ \varrho_{3}(\chi) & \phi\end{array}\right)$ with a coefficient of proportionality which is non-zero even in the $\lambda_{j} \rightarrow-1$ limit. Then if we write

$$
\Xi(\chi)=\sum_{b(\psi) \leqq s} T_{\chi \psi} \Theta(\psi),
$$

it follows from Theorem 6.2.8 that $T_{\chi \psi}$ is triangular and that the "diagonal elements" $T_{\chi \mu(\chi)}$ are non-zero for all $\lambda$. Thus $T^{-1}$ exists and is continuous in $\lambda$ (in particular, at $\lambda_{j}=-1$ ); this implies the lemma.

Remark 6.2.12. It follows from Lemma 6.2.11 that $\{\Theta(\psi) \mid b(\psi) \leqq s\}$ span the vector space $V(N, s)$ in the $\lambda_{j} \rightarrow-1$ limit, and that the action of the fundamental group on the $\{\Theta(\psi)\}$ is given by specializing the $\lambda$ 's in the generic formulae (4.5.8) and (4.5.9), i.e.

$$
\mathscr{L}(a(\mathscr{S})) \Theta(\psi)=v(\mathscr{S})_{\psi} \sum_{b(\eta) \leqq s} u(\mathscr{S})_{\eta} \Theta(\eta) .
$$

To complete the analysis it remains only to determine the linear relations among the $\Theta$ 's in the limit, and hence the dimension of $V(N, s)$. We first observe that the Landau curves $L(\chi)$, with $|\chi|=s=m+1$, are poles, and that therefore the vectors $u\left(\begin{array}{cc}\chi & \varrho_{4}(\chi) \\ \varrho_{3}(\chi) & \phi\end{array}\right)$ must vanish.

Lemma 6.2.13. The linear relations

$$
\sum_{b(\psi) \leqq s} u\left(\begin{array}{cc}
\chi & \varrho_{4}(\chi) \\
\varrho_{3}(\chi) & \phi
\end{array}\right)_{\psi} \Phi(\psi),
$$


with $|\chi|=s, \chi \subset \Omega_{N}$, are independent. Moreover, they enable us to express each vector $\Theta(\psi), b(\psi)=s$, as a linear combination of vectors $\Theta\left(\psi^{\prime}\right)$, $b\left(\psi^{\prime}\right)<s$.

Proof. This follows from the triangularity properties established in Theorem 6.2.8.

We need one more combinatoric result:

Lemma 6.2.14. For any $\chi \subset \Omega$ with $|\chi| \leqq s, N \in \chi$, we have

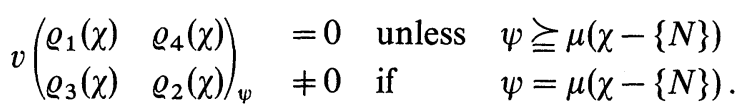

Then we have finally

Theorem 6.2.15. The vectors $\{\Theta(\psi) \mid b(\psi)<s\}$ form a basis for $V(N, s)$.

Proof. We know from Lemma 6.2.13 that these vectors span $V(N, s)$. Now suppose they are linearly dependent:

$$
\Phi=\sum_{b(\psi)<s} c_{\psi} \Theta(\psi)=0,
$$

with not all coefficients $c_{\psi}=0$. From the triangularity established in Lemma 6.2.14 there is a signature $\mathscr{S}=\left(\begin{array}{ll}\varrho_{1}(\chi) & \varrho_{4}(\chi) \\ \varrho_{3}(\chi) & \varrho_{2}(\chi)\end{array}\right)$ of the type considered there such that $(v(\mathscr{S}), \Phi) \neq 0$. From Lemma 5.2.9 this means that the curve $L(\chi)$ is singular on $\Phi$, and hence that $\Phi \neq 0$.

Corollary 6.2.16. The dimension of $V(N, s)$ is $d(N, s-1)=d(N, m)$.

We note that $d(N, m)$ follows as an upper bound for the dimension of $V(N, s)$ from the homology decomposition theorem of Fotiadi et al. [15].

\section{Appendix A: The Integration Cycle $\gamma_{0}$}

In this appendix we study the behavior of the integral (2.5) taken over a certain integration contour $\gamma_{0}$. That is, writing

we study

$$
G(B, x)=\frac{P(x) \prod_{j=1}^{r}\left[l_{j}(x)\right]^{\lambda_{j}}}{[B(x)]^{\mu}},
$$

$$
H_{\gamma_{0}}(B)=\int_{\gamma_{0}} G(B, x)^{s} \eta(x) .
$$

The contour $\gamma_{0}$ is such that $H_{\gamma_{0}}\left(B_{0}\right)$ is well defined. Assume that the signs of the linear forms $l_{j}$ are chosen so that, for some $Y \in \mathbb{P}^{s}, l_{j}(Y)>0, j=1, \ldots r$. 
Then we have

Theorem A. 2. Let $\mathscr{S}=\left(\begin{array}{ll}\varrho_{1} & \varrho_{4} \\ \varrho_{3} & \varrho_{2}\end{array}\right)$ be a signature for (A.1), let a $(\mathscr{S})$ $\in \mathbb{C}(\mathscr{G}(r, s))$ be the corresponding discontinuity operator, and let $\gamma_{0}$ be given as in Definition A.7 below. Then

$$
\mathscr{L}(a(\mathscr{S}))\left[H_{\gamma_{0}}\left(B_{0}\right)\right]=0
$$

iff $\varrho_{3}$ and $\varrho_{4}$ are non-empty.

Corollary A. 3. $H_{\gamma_{0}}$ is singular on every curve $M(\chi)$.

Proof. From Lemma 3.3.9 we see that for any $\chi$ with $|\chi| \leqq s$ there is a signature $\mathscr{S}=\left(\begin{array}{ll}\varrho_{1} & \chi \\ \phi & \varrho_{2}\end{array}\right)$. Then Theorem A.2 implies $a(\mathscr{S}) H_{\gamma_{0}} \neq 0$.

We begin with several preliminary results

Lemma A. 4. Let $B_{2} \in M(\chi)$ be a real non-singular point of $M$, let $B_{0}$, $B_{1}$ be positive definite, and let $\alpha_{0}, \alpha_{1}$ be the elementary loops around $B_{2}$ based on $B_{0}$ and $B_{1}$ respectively, defined using the anti-clockwise convention. Let $\beta(t), 0 \leqq t \leqq 1$, be a path from $B_{0}$ to $B_{1}$, with $\beta(t)$ positive definite for all $t$. Then $\alpha_{0}=\beta^{-1} \alpha_{1} \beta$.

Proof. This follows immediately from Lemma 3.3.2.

Now choose a point $Y \in \mathbb{P}^{s}$ such that $l_{j}(Y)>0, j=1, \ldots r$. We take coordinates in which $Y=(0, \ldots 0,1)$; with this choice we write any point $x \in \mathbb{P}^{s}$ in the form $x=\left(y, x_{s+1}\right)$, with $y=\left(y_{1}, \ldots y_{s}\right)$. Let $A$ be a positive definite form on $\mathbb{C}^{s}$, and define the quadratic form $B(A, R)$ on $\mathbb{P}^{s}$ by

$$
B(A, R)(x)=A(y)-R x_{s+1}^{2} .
$$

In suitable coordinates, we have

$$
B(A, R)=\sum_{1}^{s} a_{i} y_{i}^{2}-R x_{s+1}^{2}, \quad a_{i}>0 .
$$

Let $A_{0}(y)=\sum_{1}^{s} y_{i}^{2}$, so that $B_{0}=B\left(A_{0},-1\right)$.

Now we may assume that the hyperplane $\left\{x_{s+1}=0\right\}$ is in general position with respect to the surfaces $\left\{l_{j}(x)=0\right\}$. The following lemma is easily verified.

Lemma A. 6. Take $\chi \subset \Omega$ with $|\chi| \leqq s$, and $x \in l(\chi)$ with $x_{s+1} \neq 0$. Then for any $\eta \subset \chi$ with $\eta \neq \Phi$ there is a $B(A, R)$ of the form (A.5), with $R>0$, satisfying

$$
\begin{gathered}
B(A, R)(x)=0 \\
\frac{\partial A(y)}{\partial y_{i}}+\sum_{j \in \chi} \tau_{j} l_{j i}=0, \quad(i=1, \ldots s),
\end{gathered}
$$

with $\tau_{j}>0$ for $j \in \eta$ and $\tau_{j}<0$ for $j \in \chi-\eta$. 
We now turn to the definition of $\gamma_{0}$. Note that an acceptable contour for the integral (A.1) is any closed contour on which $G$ is single valued. We usually specify such an object by several $s$ (real)-dimensional cells, with identifications along their boundaries, and a determination of $G$ on each. The values of $G$ on two identified boundaries must agree.

Definition A. 7. Take $0<\varepsilon \ll 1$, and define $f: \mathbb{R} \rightarrow \mathbb{R}$ by

$$
f(u)= \begin{cases}\varepsilon & \text { if }|u| \leqq 1 \\ 1+\varepsilon-|u| & \text { if }|u|>1 .\end{cases}
$$

For $A$ as above, and $R<0$, define a contour $\gamma(A, R)$ to consist of two cells,

$$
\begin{aligned}
c_{ \pm}=\left\{\left([i \pm f(u)]\left[-R / a_{1}\right]^{\frac{1}{2}} z_{1}, \ldots[i \pm f(u)]\left[-R / a_{s}\right]^{\frac{1}{2}} z_{s}, 1\right) \mid\right. \\
\left.z_{1}, \ldots z_{s} \in \mathbb{R}, u=\sum_{1}^{s} z_{i}^{2}, \text { and } u \leqq 1+\varepsilon\right\},
\end{aligned}
$$

joined along their common boundary $u=1+\varepsilon$. Note $Y \in c_{+} \cap c_{-}$; we choose determinations $G_{ \pm}(B(A, R), Y)$ on $c_{ \pm}$which satisfy

$$
G_{+}=e^{2 \pi i \mu} G_{-}
$$

and which depend continuously on $A$.

Now it is easily verified that $H_{\gamma(A, R)}(B(A, R))$ is analytic at all points $B(A, R)$ with $R<0$. We define $\gamma_{0}=\gamma\left(A_{0},-1\right)$. A typical contour $\gamma(A, R)$ is shown in Fig. 10 for the case $s=1$.

We now turn to Theorem A.2. We will sketch the proof of this theorem only for certain signatures (the proof for the remaining signatures is somewhat more complicated). However, the results which we discuss suffice for Corollary A.3 and for the applications of $\S 6$.

Case 1. The leading curve $M(\phi)$.

For $M(\phi)$ we prove Theorem A.2 only for the signature $\mathscr{S}=\left(\begin{array}{ll}\Omega & \phi \\ \phi & \phi\end{array}\right)$. Consider the line in the parameter space $\{B(A, R) \mid R \in \mathbb{C}\}$. The point $B(A, 0)$ in this line is on $M(\mathscr{S})$, and the cycle $\gamma(A, R)$, for $R<0$, is the vanishing cycle for this singularity. In particular $\gamma_{0}$ is the vanishing cycle; thus $a(\mathscr{S}) H_{\gamma_{0}} \neq 0$.

Case 2. The curve $M(\chi),|\chi| \geqq 1$.

Let $x=(y, 1)$ be an arbitrary point of $l(\chi)$ not on $\left\{x_{s+1}=0\right\}$; let $\varrho_{1}=\left\{j \mid l_{j}(x)>0\right\}, \varrho_{2}=\left\{j \mid l_{j}(x)<0\right\}$. We will prove Theorem A.2 for an arbitrary signature $\mathscr{S}=\left(\begin{array}{ll}\varrho_{1} & \varrho_{4} \\ \varrho_{3} & \varrho_{2}\end{array}\right)$ with $\varrho_{3} \neq \phi$. 


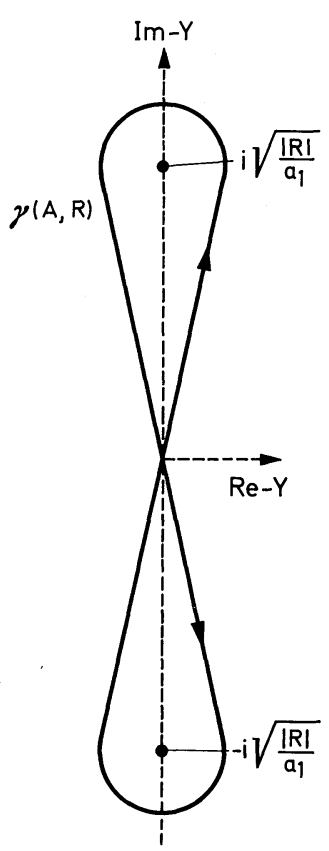

Fig. 10. The contour $\gamma(A, R)$

Now by Lemma A.6 there is a quadratic form $A$ and a positive number $R_{0}$ such that $B\left(A, R_{0}\right) \in M(\mathscr{S})$. Consider the line in the parameter space given by $\{B(A, R) \mid R \in \mathbb{C}\}$, and construct an elementary loop $\{B(A, R(t)) \mid 0 \leqq t \leqq 1\}$ around $B\left(A, R_{0}\right)$, with $R(0)=R(1)=-1$, using the anticlockwise convention. Using Lemma A.4, we see that we wish to prove that the discontinuity of $H_{\gamma(A,-1)}(B(A,-1))$, when continued around this loop, is zero if $\varrho_{4} \neq \phi$.

We will give only a brief sketch of this proof (the details are complicated and unilluminating). Note that for some $t_{0}, 0<t_{0}<1$, we have $R\left(t_{0}\right)>R_{0}$. One constructs (fairly explicitly) a contour $\delta(t)\left(0 \leqq t \leqq t_{0}\right)$, with $\delta(0)=\gamma(A,-1)$, in such a way that $H_{\delta(t)}(B(A, R(t)))$ is the analytic continuation of $H_{\gamma(A,-1)}$ along the path $B(A, R(t))$. Now for $B=B\left(A, R\left(t_{0}\right)\right)$ the vanishing cycle for $M(\mathscr{S})$ is

$$
\begin{aligned}
e(\mathscr{S})=\left\{x \in \mathbb{P}^{s} \mid l_{j}(x)>0\right. & \text { if } j \in \varrho_{4}, \\
l_{j}(x)<0 \quad \text { if } j \in \varrho_{3}, & \text { and } B(x)<0\} .
\end{aligned}
$$

One may calculate explicitly the Kronecker index of $e(\mathscr{S})$ and $\delta\left(t_{0}\right)$ and show that it vanishes if $\varrho_{4} \neq \phi$. This completes the proof. 


\section{Appendix B. The Word at Infinity in $\mathscr{G}(N, N-1)$}

In this appendix we derive an explicit expression for the word at infinity in the group $\mathscr{G}(N, N-1)$, the group used to study analytic properties when there are no second kind singularities (cf. 5.2). Recall that the word at infinity $w_{\infty}$ is the group element defined by a loop in any generic line which circles the point at infinity counterclockwise, or, equivalently, circles all finite singular points clockwise.

Take any $i \in \Omega$, and consider signatures of the form

$$
\mathscr{S}=\left(\begin{array}{ll}
\eta_{1} \cup\{i\} & \phi \\
\eta_{3} & \eta_{2}
\end{array}\right)
$$

with $\eta_{1} \subset\{k: k<i\}, \eta_{2} \subset\{k: k>i\}$, and $\bigcup_{j=1}^{3} \eta_{j}=\Omega_{i}$. We define an integer valued function $p_{i}$ on these signatures by

$$
p_{i}(\mathscr{S})=\left|\eta_{2}\right|-\left|\eta_{1}\right|+\left|\eta_{3} \cap\{k: k<i\}\right|-\left|\eta_{3} \cap\{k: k>i\}\right| .
$$

Lemma B. 2. The element $\beta_{i}=\alpha\left(\begin{array}{ll}i & \phi \\ \phi & \phi\end{array}\right)$ is given by any product of the $2^{N-1}$ elements $\alpha(\mathscr{S})$, where $\mathscr{S}$ is of the form (B.1), in which the order of the factors is such that $p_{i}\left(\mathscr{S}_{1}\right)<p_{i}\left(\mathscr{S}_{2}\right)$ implies that $\alpha\left(\mathscr{S}_{1}\right)$ appears to the left of $\alpha\left(\mathscr{S}_{2}\right)$ in the product.

Proof. One obtains an expansion of $\beta_{i}$ as a product of the indicated elements by repeated application of Definition 3.3.21. Then it may be checked that the order of any two noncommuting factors is uniquely and correctly determined by the stated rule.

Theorem B. 3. The word at infinity in $\mathscr{G}(N, N-1)$ is given by

$$
w_{\infty}^{-1}=\beta_{1} \ldots \beta_{N} .
$$

Proof. We consider the generic line $B(t)=B_{0}^{\prime}-t B^{\prime}$, where the matrix $B(t)$ is given by $B(t)_{j j}=1-t b_{j}, B(t)_{j k}=\varepsilon_{j k}$ for $j \neq k$, and the real numbers $b_{j}, \varepsilon_{j k}$ satisfy

$$
\begin{gathered}
0<b_{j}<b_{k}, \quad \forall j<k, \\
\varepsilon_{j k}=\varepsilon_{k j}<0, \\
\left|\varepsilon_{j k}\right| \ll 1 .
\end{gathered}
$$

(Note that the base point $B_{0}^{\prime}$ is in the Euclidean region and so may be used in place of $B_{0}$ (cf. Lemma A.4).) This line intersects $M(\chi)$ in $\left|\chi^{\prime}\right|$ 
points given by $t=t_{i}(\chi), i \in \chi^{\prime}$, where

$$
\begin{array}{ll}
t_{i}(\chi)=b_{i}^{-1}+\sum_{j \in \chi^{\prime}-\{i\}} \frac{\varepsilon_{i j}^{2}}{b_{j}-b_{i}}+O\left(\varepsilon^{3}\right), & \text { if } \quad\left|\chi^{\prime}\right|>1 ; \\
t_{i}(\chi)=b_{i}^{-1} & \text { if } \quad \chi^{\prime}=\{i\} .
\end{array}
$$

Moreover, at $t=t_{i}(\chi)$,

$$
\begin{aligned}
& x_{i}\left(B\left(t_{i}(\chi)\right), \chi\right)=1+O(\varepsilon) \\
& x_{k}\left(B\left(t_{i}(\chi)\right), \chi\right)=-\varepsilon_{i k} /\left(1-b_{k} / b_{i}\right)+O\left(\varepsilon^{2}\right), \quad \forall k \in \chi^{\prime}-\{i\}, \\
& \tau_{k}\left(B\left(t_{i}(\chi)\right), \chi\right)=-2 \varepsilon_{i k}+O\left(\varepsilon^{2}\right), \quad \forall k \in \chi .
\end{aligned}
$$

From these results we can compute the signatures of the finite singular points in this line and hence the corresponding expression for $w_{\infty}^{-1}$ as a product of generators $\alpha(\mathscr{S})$. Note that these points fall into $N$ clusters near the points $b_{i}^{-1} 1 \leqq i \leqq N$ and therefore the expression for $w_{\infty}^{-1}$ may be written as a corresponding product of $N$ factors. Each of these factors is, for any choice of the $b_{j}, \varepsilon_{i k}$ satisfying (B.4), a product of the form described in Lemma B.2 for the corresponding index $i$ and hence is equal to $\beta_{i}$.

\section{References}

1. Ponzano, G., Regge, T., Speer, E. R., Westwater, M. J.: Commun. Math. Phys. 15, 83-132 (1969).

2. Ponzano, G., Regge, T.: The Monodromy Group of One-Loop Relativistic Feynman Integrals. Published in a volume on the occasion of the 60th birthday of the academician N. N. Bogoliubov.

3. Speer, E. R.: Generalized Feynman Amplitudes. Princeton, N. J.: Princeton University Press 1969.

4. Regge, T.: The Fundamental Group of Poincare and the Analytic Properties of Feynman Relativistic Amplitudes. Nobel Symposium 8; Elementary Particle Theory, ed. Nils Svartholm. New York: Interscience 1969.

5. Eden, R. J., Landshoff, P. V., Olive, D. I., Polkinghorne, J. C.: The Analytic S-Matrix. Cambridge: Cambridge University Press 1966.

6. Regge, T.: Algebraic Topology Methods in the Theory of Feynman Relativistic Amplitudes. Battelle Rencontres, 1967. Lectures in Mathematics and Physics, ed. C. M. DeWitt, J. A. Wheeler. New York: W. A. Benjamin 1968.

7. Halmos, P. R.: Finite Dimensional Vector Spaces. Princeton, N. Y.: Princeton University Press 1962.

8. Byers, N., Yang, C. N.: Rev. Mod. Phys. 36, 595 (1964).

9. van der Waerden, B. L.: Modern Algebra, Vol. II. New York: Fredrick Ungar Publishing Co. 1948.

10. Pham, F.: Introduction à l'Étude Topologique des Singularités de Landau. Paris: Gauthier-Villars 1967.

11. Westwater, M. J.: Structure of Feynman Functions. Thesis, Cambridge, 1966.

12. Plemelj, J.: Problems in the Sense of Riemann and Klein. Interscience Tracts in Mathematics 16, 1964. 
13. Schläfli, L. Theorie der vielfachen Kontinuität. Berne 1852; Ges. Math. Abh I. Basel 1950 (p. 209).

Polyà, G.: Induction and Analogy in Mathematics. Princeton, N.J.: Princeton University Press, 1954 (p. 43).

14. Boyling, J. B.: Nuovo Cimento 53, 351-375 (1968).

15. Fotiadi, D., Froissart, M., Lascoux, J., Pham, F.: Topology 4, 159-191 (1965). Reprinted in Homology and Feynman Integrals, R. C. Hwa and V. L. Teplitz. New York: W. A. Benjamin 1966.

16. Hironaka, H.: Ann. Math. 79, 109-326 (1964).

17. Fotiadi, D.: Désingularisation et Graphes de Feynman. Séminaire, Collège de France, 1969.

18. - Pham, F.: Analytic Study of Some Feynman Graphs by Homological Methods. Reprinted in Homology and Feynman Integrals, R. C. Hwa and V. L. Teplitz. New York: W. A. Benjamin 1966.

19. Garside, F. A.: Quart. J. Math. Oxford (2) 20, 235-254 (1969).

G. Ponzano

Istituto di Fisica

Universita di Torino

Torino, Italy
T. Regge, E. R. Speer, M. J. Westwater Institute for Advanced Study

Princeton, N. J. 08540, USA 